\title{
TWIST AUTOMORPHISMS ON QUANTUM UNIPOTENT CELLS AND DUAL CANONICAL BASES
}

\author{
YOSHIYUKI KIMURA AND HIRONORI OYA
}

\begin{abstract}
In this paper, we construct twist automorphisms on quantum unipotent cells, which are quantum analogues of the Berenstein-Fomin-Zelevinsky twist automorphisms on unipotent cells. We show that those quantum twist automorphisms preserve the dual canonical bases of quantum unipotent cells.

Moreover we prove that quantum twist automorphisms are described by the syzygy functors for representations of preprojective algebras in the symmetric case. This is the quantum analogue of Geiß-Leclerc-Schröer's description, and Geiß-Leclerc-Schröer's results are essential in our proof. As a consequence, we show that quantum twist automorphisms are compatible with quantum cluster monomials. The 6-periodicity of specific quantum twist automorphisms is also verified.
\end{abstract}

\section{Contents}

1. Introduction

2. Preliminaries (1) : Kac-Moody Lie algebras and associated flag schemes

3. Preliminaries (2) : Quantized enveloping algebras and canonical bases

4. Quantum unipotent cells and the De Concini-Procesi isomorphisms

5. Quantum twist isomorphisms

6. Twist automorphisms on quantum unipotent cells

7. Quantum twist automorphisms and quantum cluster algebras

8. Finite type cases : 6-periodicity

References

\section{INTRODUCTION}

1.1. Canonical bases and cluster algebras. Let $G$ be a connected, simply-connected, complex simple algebraic group with a fixed maximal torus $H$, a pair of Borel subgroups $B_{ \pm}$ with $B_{+} \cap B_{-}=H$, the Weyl group $W=\operatorname{Norm}_{G}(H) / H$ and the maximal unipotent subgroups $N_{ \pm} \subset B_{ \pm}$(In the main body of this paper, we deal with "the Kac-Moody groups"). Let $\mathbf{U}_{q}(\mathfrak{g})$ be the Drinfeld-Jimbo quantized enveloping algebra of the corresponding Lie algebra $\mathfrak{g}$, and $\mathbf{U}_{q}^{-}(\mathfrak{g})$ be its negative part which arises from the triangular decomposition of $\mathfrak{g}$. In Lus90a], Lusztig constructed the canonical bases $\mathbf{B}$ of $\mathbf{U}_{q}^{-}(\mathfrak{g})$ using perverse sheaves

The work of the first author was supported by JSPS Grant-in-Aid for Scientific Research (S) 24224001 and JSPS Grant-in-Aid for Young Scientists (B) 17K14168.

The work of the second author was supported by Grant-in-Aid for JSPS Fellows (No. 15J09231) and the Program for Leading Graduate Schools, MEXT, Japan. It was also supported by the European Research Council under the European Union's Framework Programme H2020 with ERC Grant Agreement number 647353 Qaffine, during the revision of this paper. 
on the varieties of quiver representations when $\mathfrak{g}$ is simply-laced. In Kas91, Kashiwara constructed the lower global bases $G^{\text {low }}(\mathscr{B}(\infty))$ of $\mathbf{U}_{q}^{-}(\mathfrak{g})$ in general. In simply-laced case, Lusztig Lus90b] proved that the two bases of $\mathbf{U}_{q}^{-}(\mathfrak{g})$ coincide. In this paper, we call the bases the canonical bases. The canonical bases have interesting structures; one is positivity of structure constants of multiplications and (twisted) comultiplication, and another is the combinatorial structure which is called Kashiwara crystal structure. Using the positivity of the canonical bases, Lusztig [Lus94] generalized the notion of the total positivity for reductive groups and related algebraic varieties.

Since $\mathbf{U}_{q}^{-}$has a natural non-degenerate Hopf pairing which makes it into a (twisted) selfdual bialgebra, we can consider $\mathbf{U}_{q}^{-}$as a quantum analogue of the coordinate rings $\mathbb{C}\left[N_{-}\right]$. The combinatorial structure of $\mathbf{B}^{\text {low }}$ and its dual basis $\mathbf{B}^{\text {up }}$ (with respect to the non-degenerate Hopf pairing), called the dual canonical bases, has been intensively studied by Lusztig [Lus10, Chapter 42] and Berenstein-Zelevinsky [BZ93, BZ96] (in the type A-case) and it became one of the origins of cluster algebras introduced by Fomin-Zelevinsky [FZ02].

1.2. Quantum unipotent subgroups and dual canonical bases. For a Weyl group element $w \in W$ (and a lift $\left.\dot{w} \in \operatorname{Norm}_{G}(H)\right)$, the unipotent root subgroups $N_{-}(w):=$ $N_{-} \cap \dot{w} N \dot{w}^{-1}$ and the Schubert cells $B_{+} \dot{w} B_{+} / B_{+}$in the full flag varieties $G / B_{+}$have attracted much attention in the development of the theory of total positivity for reductive groups. GeißLeclerc-Schröer [GLS11] introduced a cluster algebra structure on $\mathbb{C}\left[N_{-}(w)\right]$ using representation theory of preprojective algebras, called an additive categorification. They also proved that the dual semicanonical basis $\mathcal{S}^{*}$ is compatible with $\mathbb{C}\left[N_{-}(w)\right]$, that is, $\mathcal{S}^{*} \cap \mathbb{C}\left[N_{-}(w)\right]$ gives a basis of $\mathbb{C}\left[N_{-}(w)\right]$, and the set of cluster monomials is contained in the dual semicanonical basis $\mathcal{S}^{*}$. Here we note that we identify the coordinate rings $\mathbb{C}\left[N_{-}(w)\right]$ of the unipotent subgroups $N_{-}(w)$ as invariant subalgebras $\mathbb{C}\left[N_{-}\right]^{N_{-} \cap \dot{w} N_{-} \dot{w}^{-1}}$ fixing a splitting $N_{-} \simeq\left(N_{-} \cap \dot{w} N_{-} \dot{w}^{-1}\right) \times N_{-}(w)$ as varieties.

For a nilpotent Lie algebra $\mathfrak{n}_{-}(w)$ associated with the subgroup $N_{-}(w)$, a quantum analogue $\mathbf{U}_{q}^{-}(w)$ of the universal enveloping algebras $\mathbf{U}\left(\mathfrak{n}_{-}(w)\right)$ has been introduced by De Concini-Kac-Procesi [DKP95] and also by Lusztig [Lus10] as subalgebras of the quantized enveloping algebras $\mathbf{U}_{q}^{-}$. They are defined as subalgebras which are generated by quantum root vectors defined by Lusztig's braid group symmetry on the quantized enveloping algebras $\mathbf{U}_{q}(\mathfrak{g})$. Meanwhile they are the linear spans of their Poincaré-Birkhoff-Witt type orthogonal monomials with respect to the non-degenerate pairing on $\mathbf{U}_{q}^{-}$. In [Kim12], the first author proved that the subalgebras $\mathbf{U}_{q}^{-}(w)$ are compatible with the dual canonical bases, that is $\mathbf{B}^{\mathrm{up}} \cap \mathbf{U}_{q}^{-}(w)$ is a base of $\mathbf{U}_{q}^{-}(w)$ and the specialization of $\mathbf{U}_{q}^{-}(w)$ (using the dual canonical basis) at $q=1$ is isomorphic to the coordinate ring $\mathbb{C}\left[N_{-}(w)\right]$, hence $\mathbf{U}_{q}^{-}(w)$ is also considered as a quantum analogue of the coordinate ring $\mathbb{C}\left[N_{-}(w)\right]$ of the unipotent subgroup.

Geiß-Leclerc-Schröer GLS13] proved that $\mathbf{U}_{q}^{-}(w)$ admits a quantum cluster algebra structure in the sense of Berenstein-Zelevinsky if $\mathfrak{g}$ is symmetric via the additive categorification and Goodearl-Yakimov GY14, GY17, proved the result using the framework of quantum nilpotent algebras in the symmetrizable case. Kang-Kashiwara-Kim-Oh KKKO18 showed that the set of quantum cluster monomials is contained in the dual canonical bases via symmetric quiver Hecke algebras when $\mathfrak{g}$ is symmetric. See [KKKO18, Introduction] for the history of this topic.

1.3. Unipotent cells and cluster structure. For a pair $\left(w_{+}, w_{-}\right)$of Weyl group elements, the intersections $G^{w_{+}, w_{-}}:=B_{+} \dot{w}_{+} B_{+} \cap B_{-} \dot{w}_{-} B_{-}$are called double Bruhat cells and the 
maximal torus $H$ acts $G^{w_{+}, w_{-}}$by left (or right) multiplication. For a certain lift $\bar{w}_{-} \in G$ of $w_{-} \in W$, the intersection $B_{+} \dot{w}_{+} B_{+} \cap N_{-} \bar{w}_{-} N_{-}$is a section of the quotient $G^{w_{+}, w_{-}} \rightarrow H \backslash$ $G^{w_{+}, w_{-}}$. The unipotent cells $N_{-}^{w}:=B_{+} \dot{w}_{+} B_{+} \cap N_{-}$are special cases of reduced double Bruhat cells where $w_{-}$is the unit of $W$. The (upper) cluster structure of the double Bruhat cells and unipotent cells have been studied in details, see Berenstein-Fomin-Zelevinsky [BFZ05] (see also Geiß-Leclerc-Schröer [GLS11 and Williams [Wil13]). In fact, in [GLS11], it is shown that the coordinate ring of the unipotent subgroup has a cluster algebra structure with unlocalized frozen variables, and that the coordinate ring of the unipotent cell has a cluster algebra structure with fully localized frozen variables.

For a Weyl group element $w \in W$, Berenstein-Fomin-Zelevinsky [BFZ96] (in the type Acase) and Berenstein-Zelevinsky BZ97 (in general) introduced twist automorphisms which are automorphisms on unipotent cells $N_{-}^{w}$ for solving the factorization problems, called the Chamber Ansatz, which describe the inverse of the "toric chart" of the Schubert varieties.

In GLS11, GLS12, Geiß-Leclerc-Schröer studied the additive categorification of the twist automorphism using representation theory of preprojective algebras, where it is given by the syzygy on the Frobenius subcategory associated with $w$. They treated the coordinate ring of the unipotent cells as the localization of the coordinate rings unipotent subgroups with respect to the (unipotent) minors associated with Weyl group elements. They also introduced the "dual semicanonical bases" of the coordinate ring of the unipotent cells, using the "multiplicative property" of dual semicanonical bases.

In this paper, we study the construction of a quantum analogue of the twist automorphisms on the quantum unipotent cells, which are the "quantized coordinate rings of the unipotent cells", and its relation to the additive categorification.

1.4. Quantum unipotent cells. Quantum coordinate rings of double Bruhat cells, called quantum double Bruhat cells, are introduced by De Concini-Procesi [DP97] in the study of representation theory of quantum groups at root of unity and also intensively studied by Joseph Jos95] in the study of prime spectra of quantized coordinate ring of $G$. BerensteinZelevinsky [BZ05] conjectured that quantum double Bruhat cells admit a structure of quantum cluster algebras via quantum minors. Goodearl-Yakimov [GY16] proved the conjecture using a quantum analogue of the Fomin-Zelevinsky twist of the double Bruhat cells.

In [DP97, De Concini-Procesi studied the relation between the quantum unipotent subgroups and the quantum unipotent cells in finite type case. In Kim12, the injectivity result of De Concini-Procesi is generalized via the study of crystal bases.

Berenstein-Rupel [BR15] studied the quantum unipotent cells via the Hall algebra technique and they constructed quantum analogue of the twist maps under the conjecture concerning the quantum cluster algebra structure and they showed that the quantum twist automorphisms preserve the triangular bases (in the sense of Berenstein-Zelevinsky [BZ14]) of the quantum unipotent cells when the Weyl group element $w$ is the square of an acyclic Coxeter element $c$ with $\ell(w)=2 \ell(c)$. We note that Qin Qin16 proved that the triangular bases (=localized dual canonical bases) in the sense of [Qin17] coincide with the triangular bases in the sense of Berenstein-Zelevinsky [BZ14] when $\mathfrak{g}$ is symmetric.

1.5. Quantum unipotent cells and the dual canonical bases. Our main results in this paper are the following:

(1) We prove the De Concini-Procesi isomorphisms between the localizations $\mathbf{A}_{q}\left[N_{-}(w) \cap \dot{w} G_{0}^{\min }\right]$ of the quantum unipotent subgroups $\mathbf{A}_{q}\left[N_{-}(w)\right]$ and the quantum unipotent cells 
$\mathbf{A}_{q}\left[N_{-}^{w}\right]$ for arbitrary symmetrizable Kac-Moody cases (Theorem 4.13). The quantum cluster structure on the quantum unipotent cells can be proved as a corollary of the existence of the De Concini-Procesi isomorphisms (Corollary [7.20).

We should remark that the original De Concini-Procesi isomorphisms [DP97, Theorem 3.2] were given under the assumption that $\mathfrak{g}$ is of finite type. In [DP97, their existence was proved by downward induction on the length of elements of the Weyl group $W$ from the longest element, which exists only in finite type cases.

(2) We introduce a quantum analogue $\gamma_{w}$ of the twist isomorphism between the unipotent cells $N_{-}^{w}$ and $N_{-}(w) \cap \dot{w} G_{0}^{\text {min }}$ which is defined using the Gauss decomposition (Theorem 5.19).

(3) We introduce a quantum analogue of the twist automorphism of unipotent cells on the quantum coordinate ring $\mathbf{A}_{q}\left[N_{-}^{w}\right]$ of the unipotent cells (without referring the quantum cluster algebra structure) and show that the quantum twist preserves the dual canonical bases (Theorem 6.1). In fact, we introduce a quantum analogue of the twist automorphism as a composite of the De Concini-Procesi isomorphism and the quantum twist isomorphism. The result that the dual canonical bases are preserved under the twist automorphism from 2 is proved as a consequence of the properties of two isomorphisms and the dual canonical bases. We note that our construction is independent of the construction by Berenstein-Rupel [BR15].

(4) We relate the quantum twist automorphisms and the quantum cluster structure under the additive categorification (Theorem 7.25). We also prove the 6 -periodicity of the twist automorphisms associated to the longest elements of the Weyl groups in finite type cases (Theorem 8.1).

1.6. Outline of the paper. The paper is organized as follows. In section 2, we prepare the notations for Kac-Moody Lie algebras, Kac-Moody groups, and flag schemes. Moreover, we give a description of the coordinate rings of unipotent cells, and express "classical twist maps", which are defined by Berenstein-Zelevinsky [BZ97], in terms of matrix coefficients. In section 3, we give a brief review of quantum unipotent subgroups, quantum closed unipotent cells and canonical/dual canonical bases. The main result in this section is "a crystalized Kumar-Peterson identity" (Theorem 3.48). In section 4, we define the dual canonical bases of the localized quantum coordinate rings and prove the De Concini-Procesi isomorphisms under the arbitrary symmetrizable Kac-Moody setting. In section 5, a quantum analogue of the twist isomorphism is introduced. In section 6, we define a quantum analogue of the twist automorphism as a composite of the quantum twist isomorphism and the De Concini-Procesi isomorphism. In section 7, we relate the quantum twist automorphisms to the quantum cluster algebra structures via Geiß-Leclerc-Schröer's additive categorification. In section 8 , we study the periodicity of the twist automorphisms associated to the longest elements in finite type cases.

1.7. Further work. The comparison with the construction by Berenstein-Rupel [BR15] and a quantum analogue of the Chamber Ansatz will be discussed in another paper1].

There is another type of "quantum twist map" which is not an automorphism, introduced by Lenagan-Yakimov [LY15. This is a quantum analogue of the Fomin-Zelevinsky twist isomorphism [FZ99]. The authors showed that it also preserves the dual canonical basis of

\footnotetext{
${ }^{1}$ After the submission of the present paper, the paper corresponding to these topics by the second author appeared as Oya17.
} 
$\mathbf{A}_{q}\left[N_{-}(w)\right][$ KO18]. However the authors do not know any explicit relations between this quantum twist map and the quantum twist automorphisms in this paper.

1.8. Basic notation. (1) Let $k$ be a field. For a $k$-vector space $V$, set $V^{*}:=\operatorname{Hom}_{k}(V, k)$. Denote by $\langle\rangle:, V^{*} \times V \rightarrow k,(f, v) \mapsto\langle f, v\rangle$ the canonical pairing.

(2) For a $k$-algebra $\mathcal{A}$, we set $\left[a_{1}, a_{2}\right]:=a_{1} a_{2}-a_{2} a_{1}$ for $a_{1}, a_{2} \in \mathcal{A}$. An Ore set $\mathcal{M}$ of $\mathcal{A}$ stands for a left and right Ore set consisting of non-zero divisors. Denote by $\mathcal{A}\left[\mathcal{M}^{-1}\right]$ the algebra of fractions with respect to the Ore set $\mathcal{M}$. In this case, $\mathcal{A}$ is naturally a subalgebra of $\mathcal{A}\left[\mathcal{M}^{-1}\right]$. See [GW04, Chapter 6].

(3) An $\mathcal{A}$-module $V$ means a left $\mathcal{A}$-module. The action of $\mathcal{A}$ on $V$ is denoted by a.v for $a \in \mathcal{A}$ and $v \in V$. In this case, $V^{*}$ is regarded as a right $\mathcal{A}$-module by $\langle f . a, v\rangle=\langle f, a . v\rangle$ for $f \in V^{*}, a \in \mathcal{A}$ and $v \in V$.

(4) For two symbols $i, j$, the notation $\delta_{i j}$ stands for the Kronecker delta.

\section{Preliminaries (1) : KaC-Moody Lie algebras and associated flag schemes}

In this section, we fix the notation concerning (symmetrizable) Kac-Moody Lie algebras $\mathfrak{g}$ and associated Kac-Moody groups $G, G^{\text {min }}$ and (not necessarily a group) schemes $\boldsymbol{G}$. See Kashiwara [Kas89] (see also Kashiwara-Tanisaki [KT95]) for more details. In subsection 2.6. we describe the coordinate rings of unipotent cells explicitly, and review "classical twist maps", which are defined by Berenstein-Zelevinsky [BZ97], in terms of matrix coefficients.

\subsection{Kac-Moody Lie algebras and their representations.}

Definition 2.1. A root datum $\left(I, \mathfrak{h}, P,\left\{\alpha_{i}\right\}_{i \in I},\left\{h_{i}\right\}_{i \in I},(),\right)$ consists of the following data

(1) $I$ : a finite index set,

(2) $\mathfrak{h}$ : a finite dimensional $\mathbb{Q}$-vector space,

(3) $P \subset \mathfrak{h}^{*}:$ a lattice, called the weight lattice,

(4) $P^{*}=\{h \in \mathfrak{h} \mid\langle h, P\rangle \subset \mathbb{Z}\}$, called the coweight lattice, with the canonical pairing $\langle-,-\rangle: P^{*} \otimes_{\mathbb{Z}} P \rightarrow \mathbb{Z}$,

(5) $\left\{\alpha_{i}\right\}_{i \in I} \subset P$ : a subset, called the set of simple roots,

(6) $\left\{h_{i}\right\}_{i \in I} \subset P^{*}$ : a subset, called the set of simple coroots,

(7) $():, P \times P \rightarrow \mathbb{Q}:$ a $\mathbb{Q}$-valued symmetric $\mathbb{Z}$-bilinear form on $P$,

satisfying the following conditions:

(a) $\left(\alpha_{i}, \alpha_{i}\right) \in 2 \mathbb{Z}_{>0}$ for $i \in I$,

(b) $\left\langle h_{i}, \mu\right\rangle=2\left(\alpha_{i}, \mu\right) /\left(\alpha_{i}, \alpha_{i}\right)$ for $\mu \in P$ and $i \in I$,

(c) $A=\left(\left\langle h_{i}, \alpha_{j}\right\rangle\right)_{i, j \in I}$ is a symmetrizable generalized Cartan matrix, that is $\left\langle h_{i}, \alpha_{i}\right\rangle=2$, $\left\langle h_{i}, \alpha_{j}\right\rangle \in \mathbb{Z}_{\leq 0}$ for $i \neq j$ and $\left\langle h_{i}, \alpha_{j}\right\rangle=0$ is equivalent to $\left\langle h_{j}, \alpha_{i}\right\rangle=0$,

(d) $\left\{\alpha_{i}\right\}_{i \in I} \subset \mathfrak{h}^{*},\left\{h_{i}\right\}_{i \in I} \subset \mathfrak{h}$ are linearly independent subsets.

The $\mathbb{Z}$-submodule $Q=\sum_{i \in I} \mathbb{Z} \alpha_{i} \subset P$ is called the root lattice, $Q^{\vee}=\sum_{i \in I} \mathbb{Z} h_{i} \subset P^{*}$ is called the coroot lattice. We set $Q_{+}=\sum_{i \in I} \mathbb{Z}_{\geq 0} \alpha_{i} \subset Q$ and $Q_{-}=-Q_{+}$. For $\xi=\sum_{i \in I} \xi_{i} \alpha_{i} \in Q$, we set ht $(\xi)=\sum_{i \in I} \xi_{i} \in \mathbb{Z}$. Let $P_{+}:=\left\{\lambda \in P \mid\left\langle h_{i}, \lambda\right\rangle \in \mathbb{Z}_{\geq 0}\right.$ for all $\left.i \in I\right\}$ and we assume that there exists $\left\{\varpi_{i}\right\}_{i \in I} \subset P_{+}$such that $\left\langle h_{i}, \varpi_{j}\right\rangle=\delta_{i j}$. Set $\rho:=\sum_{i \in I} \varpi_{i} \in P_{+}$.

The quadruple $\left(\mathfrak{h},\left\{\alpha_{i}\right\}_{i \in I},\left\{h_{i}\right\}_{i \in I},(),\right)$ is called a realization of $A$. Let $\mathfrak{g}$ be the associated Kac-Moody Lie algebra, that is, the Lie algebra $\mathfrak{g}$ over $\mathbb{C}$ which is generated by $\left\{e_{i}, f_{i} \mid i \in\right.$ $I\} \cup \mathfrak{h}$ with the following relations:

$(1) \mathfrak{h}$ is a vector subspace of $\mathfrak{g}$, 
(2) $\left[h, h^{\prime}\right]=0$ for $h, h^{\prime} \in \mathfrak{h}$,

(3) $\left[h, e_{i}\right]=\left\langle h, \alpha_{i}\right\rangle e_{i}$ and $\left[h, f_{i}\right]=-\left\langle h, \alpha_{i}\right\rangle f_{i}$ for $h \in \mathfrak{h}$ and $i \in I$,

(4) $\left[e_{i}, f_{j}\right]=\delta_{i j} h_{i}$ for $i, j \in I$,

(5) $\operatorname{ad}\left(e_{i}\right)^{1-a_{i j}}\left(e_{j}\right)=\operatorname{ad}\left(f_{i}\right)^{1-a_{i j}}\left(f_{j}\right)=0$ for $i, j \in I$ with $i \neq j$, where $\operatorname{ad}(x)(y)=[x, y]$.

Let $\mathfrak{n}_{+}\left(\right.$resp. $\left.\mathfrak{n}_{-}\right)$be the Lie subalgebra of $\mathfrak{g}$ generated by $\left\{e_{i} \mid i \in I\right\}$ (resp. $\left\{f_{i} \mid i \in I\right\}$ ). Then we have $\mathfrak{g}=\mathfrak{n}_{-} \oplus \mathfrak{h} \oplus \mathfrak{n}_{+}$, and it is called a triangular decomposition of $\mathfrak{g}$. Let $\mathfrak{p}_{i}^{+}=\mathfrak{n}_{+} \oplus \mathfrak{h} \oplus \mathbb{C} f_{i}$ and $\mathfrak{p}_{i}^{-}=\mathfrak{n}_{-} \oplus \mathfrak{h} \oplus \mathbb{C} e_{i}$.

Let $\mathfrak{g}=\bigoplus_{\alpha \in \mathfrak{h}^{*}} \mathfrak{g}_{\alpha}$ be its root space decomposition, $\Delta=\left\{\alpha \in \mathfrak{h}^{*} \mid \mathfrak{g}_{\alpha} \neq 0\right\} \backslash\{0\}$ be the set of roots, and $\Delta_{ \pm}$be the subsets of positive and negative roots. For a Lie algebra $\mathfrak{s}$, its universal enveloping algebra is denoted by $\mathbf{U}(\mathfrak{s})$.

Let $W$ be the Weyl group associated with the above root datum, that is the subgroup of $G L\left(\mathfrak{h}^{*}\right)$ which is generated by simple reflections $\left\{s_{i}\right\}_{i \in I}$, where

$$
s_{i}(\mu)=\mu-\left\langle h_{i}, \mu\right\rangle \alpha_{i}\left(\mu \in \mathfrak{h}^{*}\right),
$$

and $\ell: W \rightarrow \mathbb{Z}_{\geq 0}$ be the length function, that is $\ell(w)$ is the smallest integer such that there exists $i_{1}, \ldots, i_{\ell} \in I$ with $w=s_{i_{1}} s_{i_{2}} \ldots s_{i_{\ell}}$. For $w \in W$, set

$$
I(w):=\left\{\boldsymbol{i}=\left(i_{1}, \ldots, i_{\ell(w)}\right) \in I^{\ell(w)} \mid w=s_{i_{1}} \cdots s_{i_{\ell(w)}}\right\} .
$$

An element of $I(w)$ is called a reduced word of $w$.

Let $\Delta^{\text {re }}:=W\left\{\alpha_{i}\right\}_{i \in I} \subset \Delta$ be the set of real roots and we set $\Delta_{ \pm}^{\text {re }}:=\Delta_{ \pm} \cap \Delta^{\text {re }}$.

Definition 2.2. (1) For $\lambda \in P_{+}$, let $V_{\mathbb{C}}(\lambda)$ be the integrable highest weight $\mathfrak{g}$-module with highest weight vector $u_{\lambda}$ of highest weight $\lambda$.

(2) Let $\mathcal{O}_{\text {int }}(\mathfrak{g})$ be the category of integrable $\mathfrak{g}$-modules $M$ satisfying the following condition:

(1) $M=\bigoplus_{\mu \in P} M_{\mu}$ with $M_{\mu}=\{m \in M \mid h . m=\langle h, \mu\rangle m$ for all $h \in \mathfrak{h}\}$ and $\operatorname{dim} M_{\mu}<\infty$ for $\mu \in P$,

(2) there exists finitely many $\lambda_{1}, \cdots, \lambda_{k} \in P_{+}$such that $P(M):=\left\{\mu \in P \mid M_{\mu} \neq 0\right\} \subset$ $\bigcup_{1 \leq j \leq k}\left(\lambda_{j}+Q_{-}\right)$.

By definition, for a finitely generated (not necessarily integrable) $\mathfrak{g}$-module $M$ satisfying the condition 1 above, the condition for $M \in \mathcal{O}_{\text {int }}(\mathfrak{g})$ is equivalent to $\operatorname{dim}_{\mathbb{C}} \mathbf{U}\left(\mathfrak{p}_{\mathfrak{i}}^{+}\right) m<\infty$ for all $i \in I$ and $m \in M$. It is well-known that $\mathcal{O}_{\text {int }}(\mathfrak{g})$ is semisimple with its simple object being isomorphic to the integrable highest weight modules $\left\{V_{\mathbb{C}}(\lambda) \mid \lambda \in P_{+}\right\}$.

Let $\varphi: \mathfrak{g} \rightarrow \mathfrak{g}$ be the anti-involution defined by $\varphi\left(e_{i}\right)=f_{i}, \varphi\left(f_{i}\right)=e_{i}, \varphi(h)=h$ for $i \in I$ and $h \in \mathfrak{h}$. For $M \in \mathcal{O}_{\text {int }}(\mathfrak{g})$, we denote by $\mathbf{D}_{\varphi} M$ the $\mathfrak{g}$-module $\bigoplus_{\mu \in M} \operatorname{Hom}\left(M_{\mu}, \mathbb{C}\right)$ whose $\mathfrak{g}$-module structure is given by

$$
\langle x . f, m\rangle=\langle f, \varphi(x) . m\rangle \text { for } x \in \mathfrak{g} \text { and } m \in M .
$$

We note that $\mathbf{D}_{\varphi} M \in \mathcal{O}_{\text {int }}(\mathfrak{g})$. For a $\mathfrak{g}$-module $M$, we denote by $M^{\mathrm{r}}$ the $\mathfrak{g}^{\text {op}}$-module $\left\{m^{\mathrm{r}} \mid m \in M\right\}$ whose $\mathfrak{g}^{\mathrm{op}}$-module structure is given by

$$
x .\left(m^{\mathrm{r}}\right)=(\varphi(x) . m)^{\mathrm{r}} \text { for } x \in \mathfrak{g} \text { and } m \in M .
$$

We denote by $\mathcal{O}_{\text {int }}^{\mathrm{r}}(\mathfrak{g})$ be the category of integrable $\mathfrak{g}^{\text {op }}$-modules $M^{\mathrm{r}}$ such that $M \in \mathcal{O}_{\text {int }}(\mathfrak{g})$. We interpret the category of $\mathfrak{g}^{\mathrm{op}}$-modules as the category of right $\mathbf{U}(\mathfrak{g})$-modules. 
2.2. (Pro-) unipotent subgroups. A subset $\Theta$ of $\Delta_{ \pm}$is called closed (resp. an ideal) if it satisfies $(\Theta+\Theta) \cap \Delta_{ \pm} \subset \Theta$ (resp. $\left.\left(\Theta+\Delta_{ \pm}\right) \cap \Delta_{ \pm} \subset \Theta\right)$. For a closed subset (resp. an ideal) $\Theta \subset \Delta_{ \pm}, \mathfrak{n}_{ \pm}(\Theta):=\bigoplus_{\alpha \in \Theta} \mathfrak{g}_{\alpha}$ is a Lie subalgebra (resp. an ideal) of $\mathfrak{n}_{ \pm}$.

Example 2.3. (1) For a Weyl group element $w \in W$, the subsets $\Delta_{ \pm}(\leq w):=\Delta_{ \pm} \cap w \Delta_{\mp}$ and $\Delta_{ \pm}(>w):=\Delta_{ \pm} \cap w \Delta_{ \pm}$are closed. Let $\mathfrak{n}_{ \pm}(\leq w):=\mathfrak{n}_{ \pm}\left(\Delta_{ \pm}(\leq w)\right)$ and $\mathfrak{n}_{ \pm}(>w):=$ $\mathfrak{n}_{ \pm}\left(\Delta_{ \pm}(>w)\right)$ be the corresponding subalgebras. We have direct sum decompositions $\mathfrak{n}_{ \pm}=$ $\mathfrak{n}_{ \pm}(\leq w) \oplus \mathfrak{n}_{ \pm}(>w)$ for $w \in W$. For a simple reflection $s_{i}$, we have $\Delta_{+} \cap s_{i} \Delta_{-}=\left\{\alpha_{i}\right\}$ and $\Delta_{+} \cap s_{i} \Delta_{+}=\Delta_{+} \backslash\left\{\alpha_{i}\right\}$. Hence we have direct sum decompositions $\mathfrak{n}_{ \pm}=\mathfrak{g}_{ \pm \alpha_{i}} \oplus \mathfrak{n}_{i}^{ \pm}$, where $\mathfrak{n}_{i}^{+}=\mathfrak{n}_{+}\left(\Delta_{+} \backslash\left\{\alpha_{i}\right\}\right)$ and $\mathfrak{n}_{i}^{-}=\mathfrak{n}_{-}\left(\Delta_{-} \backslash\left\{-\alpha_{i}\right\}\right)$.

(2) For $k \in \mathbb{Z}_{\geq 0}$, we set $\Delta_{ \pm}^{\geq k}:=\left\{\alpha \in \Delta_{ \pm} \mid \pm\right.$ht $\left.(\alpha) \geq k\right\}$ and $\mathfrak{n}_{ \pm}^{\geq k}:=\mathfrak{n}_{ \pm}\left(\Delta_{ \pm}^{\geq k}\right)$. Then we have $\left(\Delta_{ \pm}^{\geq k}+\Delta_{ \pm}\right) \cap \Delta_{ \pm} \subset \Delta_{ \pm}^{\geq k}$. Hence $\mathfrak{n}_{ \pm}^{\geq k}$ is an ideal of $\mathfrak{n}_{ \pm}$.

It is clear that $\mathfrak{n}_{ \pm} / \mathfrak{n}_{ \pm}^{\geq k}$ is a finite dimensional nilpotent Lie algebra. We set

$$
\hat{\mathfrak{n}}_{ \pm}=\lim _{\longleftarrow} \mathfrak{n}_{ \pm} / \mathfrak{n}_{ \pm}^{\geq k}=\prod_{\alpha \in \Delta_{ \pm}} \mathfrak{g}_{\alpha}
$$

Let $\boldsymbol{N}_{ \pm}$be the pro-unipotent group scheme whose pro-nilpotent pro-Lie algebra is $\hat{\mathfrak{n}}_{ \pm}$that is defined by

$$
N_{ \pm}=\lim _{\longleftarrow} \exp \left(\mathfrak{n}_{ \pm} / \mathfrak{n}_{ \pm}^{\geq k}\right)=\operatorname{Spec}\left(\mathbf{U}\left(\mathfrak{n}_{ \pm}\right)_{\mathrm{gr}}^{*}\right)
$$

where $\exp \left(\mathfrak{n}_{ \pm} / \mathfrak{n}_{ \pm}^{\geq k}\right)$ is an unipotent algebraic group whose Lie algebra is the nilpotent Lie algebra $\mathfrak{n}_{ \pm} / \mathfrak{n}_{ \pm}^{\geq k}$ and $\mathbf{U}\left(\mathfrak{n}_{ \pm}\right)_{\text {gr }}^{*}$ is the graded dual of $\mathbf{U}\left(\mathfrak{n}_{ \pm}\right)$with respect to the natural $Q_{ \pm^{-}}$ grading on $\mathbf{U}\left(\mathfrak{n}_{ \pm}\right)$(the degrees of $e_{i}$ and $f_{i}$ are $\alpha_{i}$, and $-\alpha_{i}$, respectively). Note that the commutative algebra structure of $\mathbf{U}\left(\mathfrak{n}_{ \pm}\right)_{\mathrm{gr}}^{*}$ is induced from the cocommutative usual coalgebra structure of $\mathbf{U}\left(\mathfrak{n}_{ \pm}\right)$. Then we have $\mathbb{C}\left[\boldsymbol{N}_{ \pm}\right]=\mathbf{U}\left(\mathfrak{n}_{ \pm}\right)_{\text {gr }}^{*}$. It is known that there exists an isomorphism of $\mathbb{C}$-schemes Exp: $\widehat{\mathfrak{n}}_{ \pm} \rightarrow \boldsymbol{N}_{ \pm}$.

For a subset $\Theta$ of $\Delta_{+}$(resp. $\left.\Delta_{-}\right)$, we set

$$
\widehat{\mathfrak{n}}_{ \pm}(\Theta):=\prod_{\alpha \in \Theta} \mathfrak{g}_{\alpha}, \quad \boldsymbol{N}_{ \pm}(\Theta):=\operatorname{Exp}\left(\widehat{\mathfrak{n}}_{ \pm}(\Theta)\right) .
$$

Then $\boldsymbol{N}_{ \pm}(\Theta)$ is a closed subgroup of $\boldsymbol{N}_{ \pm}$if $\Theta$ is closed and is a normal subgroup of $\boldsymbol{N}_{ \pm}$if $\Theta$ is an ideal. Let $N_{ \pm} \subset \boldsymbol{N}_{ \pm}$be the subgroup which is generated by $\left\{\boldsymbol{N}_{ \pm}( \pm \alpha) \mid \alpha \in \Delta_{+}^{\mathrm{re}}\right\}$, which has an ind-group scheme structure.

For a Weyl group element $w \in W$ and $i \in I$, let

$$
N_{ \pm}(w):=\boldsymbol{N}_{ \pm}\left(\Delta_{ \pm}(\leq w)\right), \quad \boldsymbol{N}_{ \pm}^{\prime}(w):=\boldsymbol{N}_{ \pm}\left(\Delta_{ \pm}(>w)\right), \quad \boldsymbol{N}_{i}^{ \pm}:=\boldsymbol{N}_{ \pm}^{\prime}\left(\Delta_{ \pm}\left(>s_{i}\right)\right) .
$$

Since $\Delta_{ \pm} \cap w \Delta_{\mp} \subset \Delta_{ \pm}^{\text {re }}$, we have $\boldsymbol{N}_{ \pm}\left(\Delta_{ \pm}(\leq w)\right) \subset N_{ \pm}$. In fact, $N_{ \pm}(w)$ are unipotent subgroups of $\boldsymbol{N}_{ \pm}$with $\operatorname{dim}\left(N_{ \pm}(w)\right)=\ell(w)$.

We have the following isomorphisms

$$
\begin{aligned}
\boldsymbol{N}_{ \pm} & \simeq \boldsymbol{N}_{ \pm}^{\prime}(w) \times\left(N_{ \pm}(w)\right) \\
& \simeq\left(N_{ \pm}(w)\right) \times \boldsymbol{N}_{ \pm}^{\prime}(w),
\end{aligned}
$$

as schemes, see [Kum02, Lemma 6.1.2]. We set $N_{ \pm}^{\prime}(w):=N_{ \pm} \cap \boldsymbol{N}_{ \pm}^{\prime}(w)$. We also have the decompositions $N_{ \pm} \simeq N_{ \pm}^{\prime}(w) \times N_{ \pm}(w) \simeq N_{ \pm}(w) \times N_{ \pm}^{\prime}(w)$. 
2.3. Borel subgroups and minimal parabolic subgroups. Let us fix a root datum $\left(A, P, P^{\vee},\left\{\alpha_{i}\right\}_{i \in I},\left\{h_{i}\right\}_{i \in I}\right)$ which gives a realization of $A$. Set $H:=\operatorname{Spec}(\mathbb{C}[P])$. Then $H$ is the algebraic torus whose character lattice is $P$ and whose $\mathbb{C}$-valued points are given by $\operatorname{Hom}_{\mathbb{Z}}\left(P, \mathbb{C}^{*}\right)$. Since $\mathbb{C}\left[\boldsymbol{N}_{ \pm}\right]=\mathbf{U}\left(\mathfrak{n}_{ \pm}\right)_{\text {gr }}^{*}$ are $Q(\subset P)$-graded algebras, we have $H$-actions on $\boldsymbol{N}_{ \pm}$. Moreover, since $\boldsymbol{N}_{ \pm}( \pm \alpha), \alpha \in \Delta_{+}^{\text {re }}$ are preserved by these $H$-actions, the subgroups $N_{ \pm}$ are also preserved by these $H$-actions. Let $\boldsymbol{B}_{ \pm}=H \ltimes \boldsymbol{N}_{ \pm}, B_{ \pm}=H \ltimes N_{ \pm}$be the semi-direct product groups.

For $i \in I$, let $G_{i}$ be the reductive group scheme whose Lie algebra is $\mathfrak{h} \oplus \mathbb{C} e_{i} \oplus \mathbb{C} f_{i}$ with $H$ a Cartan subgroup. Let $\gamma_{i}: S L(2, \mathbb{C}) \rightarrow G_{i}$ be the morphism of algebraic groups which is induced by the homomorphism of Lie algebras given by $e \mapsto e_{i}$ and $f \mapsto f_{i}$. For a simple reflection $s_{i}$, let $\bar{s}_{i} \in G_{i}$ and $\overline{\overline{s_{i}}} \in G_{i}$ be the lift defined by

$$
\begin{aligned}
& \overline{s_{i}}=\gamma_{i}\left(\left[\begin{array}{cc}
0 & -1 \\
1 & 0
\end{array}\right]\right)=\exp \left(-e_{i}\right) \exp \left(f_{i}\right) \exp \left(-e_{i}\right), \\
& \overline{\overline{s_{i}}}=\gamma_{i}\left(\left[\begin{array}{cc}
0 & 1 \\
-1 & 0
\end{array}\right]\right)=\exp \left(e_{i}\right) \exp \left(-f_{i}\right) \exp \left(e_{i}\right) .
\end{aligned}
$$

Let $G_{i}^{+}$(resp. $G_{i}^{-}$) be the subgroup of $G_{i}$ with $\mathfrak{h} \oplus \mathbb{C} e_{i}$ (reps. $\mathfrak{h} \oplus \mathbb{C} f_{i}$ ) as its Lie algebra. We have $G_{i}^{ \pm}=G_{i} \cap \boldsymbol{B}_{ \pm}$and isomorphism $\boldsymbol{B}_{ \pm}=G_{i}^{ \pm} \times \boldsymbol{N}_{i}^{ \pm}$as schemes.

For $i \in I$, let $\left(\mathfrak{p}_{i}^{ \pm}, H\right)$-mod (resp. $\left(\mathfrak{p}_{i}^{ \pm}, H\right)^{\mathrm{op}}$-mod) be the category of left (resp. right) finite dimensional $P$-weighted $\mathfrak{h}$-semisimple $\mathbf{U}\left(\mathfrak{p}_{i}^{ \pm}\right)$-modules.

Let us consider the following $\mathbb{C}$-algebras:

$$
\mathbb{C}\left[\boldsymbol{P}_{i}^{ \pm}\right]:=\left\{\begin{array}{l|l}
f \in \operatorname{Hom}_{\mathbb{C}}\left(\mathbf{U}\left(\mathfrak{p}_{i}^{ \pm}\right), \mathbb{C}\right) & \begin{array}{c}
\mathbf{U}\left(\mathfrak{p}_{i}^{ \pm}\right) f \in\left(\mathfrak{p}_{i}^{ \pm}, H\right) \text {-mod } \\
f \mathbf{U}\left(\mathfrak{p}_{i}^{ \pm}\right) \in\left(\mathfrak{p}_{i}^{ \pm}, H\right)^{\mathrm{op}} \text {-mod }
\end{array}
\end{array}\right\}
$$

where we consider the $\mathbf{U}\left(\mathfrak{p}_{i}^{ \pm}\right)$-bimodule structure on $\operatorname{Hom}_{\mathbb{C}}\left(\mathbf{U}\left(\mathfrak{p}_{i}^{ \pm}\right), \mathbb{C}\right)$ defined by

$$
\langle x . f . y, u\rangle=\langle f, y . u . x\rangle\left(x, y \in \mathfrak{p}_{i}^{ \pm}, f \in \operatorname{Hom}_{\mathbb{C}}\left(\mathbf{U}\left(\mathfrak{p}_{i}^{ \pm}\right), \mathbb{C}\right), u \in \mathbf{U}\left(\mathfrak{p}_{i}^{ \pm}\right)\right) .
$$

Then the coproduct $\mathbf{U}\left(\mathfrak{p}_{i}^{ \pm}\right) \rightarrow \mathbf{U}\left(\mathfrak{p}_{i}^{ \pm}\right) \otimes \mathbf{U}\left(\mathfrak{p}_{i}^{ \pm}\right)$induces a commutative algebra structure on $\operatorname{Hom}_{\mathbb{C}}\left(\mathbf{U}\left(\mathfrak{p}_{i}^{ \pm}\right), \mathbb{C}\right)$ and $\mathbb{C}\left[\boldsymbol{P}_{i}^{ \pm}\right]$is a subalgebra of $\operatorname{Hom}_{\mathbb{C}}\left(\mathbf{U}\left(\mathfrak{p}_{i}^{ \pm}\right), \mathbb{C}\right)$. We define a schemes $\boldsymbol{P}_{i}^{ \pm}:=\operatorname{Spec}\left(\mathbb{C}\left[\boldsymbol{P}_{i}^{ \pm}\right]\right)$as spectrum. The product $\mathbf{U}\left(\mathfrak{p}_{i}^{ \pm}\right) \otimes \mathbf{U}\left(\mathfrak{p}_{i}^{ \pm}\right) \rightarrow \mathbf{U}\left(\mathfrak{p}_{i}^{ \pm}\right)$induces the morphism of schemes $\boldsymbol{P}_{i}^{ \pm} \times \boldsymbol{P}_{i}^{ \pm} \rightarrow \boldsymbol{P}_{i}^{ \pm}$and it gives the structure of group scheme on $\boldsymbol{P}_{i}^{ \pm}$and we have decomposition $\boldsymbol{P}_{i}^{ \pm} \cong G_{i} \ltimes \boldsymbol{N}_{i}^{ \pm}$and $\boldsymbol{P}_{i}^{ \pm} \supset \boldsymbol{B}_{ \pm}$for $i \in I$. See [KT95] for more details.

2.4. Kac-Moody groups and flag schemes. Let $G$ be the "maximal" Kac-Moody group over $\mathbb{C}$ completed along the positive roots which is defined in Kumar Kum02, 6.1.16] and let $G^{\text {min }} \subset G$ be the "minimal" Kac-Moody group over $\mathbb{C}$ defined in Kumar [Kum02, 7.4.1]. They satisfy $\boldsymbol{B}_{+} \subset G$ and $B_{+} \subset G^{\text {min }}$. See [Kum02] for details. We also introduce the scheme $\boldsymbol{G}_{\infty}$ and its open subscheme $\boldsymbol{G}$ following Kashiwara [Kas89] (see also Kashiwara-Tanisaki [KT95])

We define the scheme $\boldsymbol{G}_{\infty}:=\operatorname{Spec}\left(\mathbf{R}_{\mathbb{C}}(\mathfrak{g})\right)$ as the spectrum of the ring of "strongly regular functions" introduced by Kac-Peterson [KP83, that is

$$
\mathbf{R}_{\mathbb{C}}(\mathfrak{g}):=\left\{f \in \operatorname{Hom}_{\mathbb{C}}(\mathbf{U}(\mathfrak{g}), \mathbb{C}) \mid \begin{array}{l}
\mathbf{U}(\mathfrak{g}) f \in \mathcal{O}_{\text {int }}(\mathfrak{g}) \\
f \mathbf{U}(\mathfrak{g}) \in \mathcal{O}_{\text {int }}^{\mathrm{r}}(\mathfrak{g})
\end{array}\right\},
$$

where we consider the bimodule structure on $\operatorname{Hom}_{\mathbb{C}}(\mathbf{U}(\mathfrak{g}), \mathbb{C})$ defined by

$$
\langle x . f . y, u\rangle=\langle f, y . u . x\rangle\left(x, y \in \mathfrak{g}, f \in \operatorname{Hom}_{\mathbb{C}}(\mathbf{U}(\mathfrak{g}), \mathbb{C}), u \in \mathbf{U}(\mathfrak{g})\right) .
$$


Let

$$
\Phi=\sum_{\lambda \in P_{+}} \Phi_{\lambda}: \bigoplus_{\lambda \in P_{+}} V_{\mathbb{C}}(\lambda)^{\mathrm{r}} \otimes V_{\mathbb{C}}(\lambda) \rightarrow \mathbf{R}_{\mathbb{C}}(\mathfrak{g})
$$

be the map defined by $\left\langle\Phi_{\lambda}\left(v_{1}^{\mathrm{r}} \otimes v_{2}\right), u\right\rangle=\left(v_{1}, u . v_{2}\right)_{\lambda}$ for $v_{1}, v_{2} \in V_{\mathbb{C}}(\lambda)$ and $u \in \mathbf{U}(\mathfrak{g})$, where $(,)_{\lambda}: V_{\mathbb{C}}(\lambda) \otimes V_{\mathbb{C}}(\lambda) \rightarrow \mathbb{C}$ is the symmetric bilinear form on $V(\lambda)$ such that $\left(u_{\lambda}, u_{\lambda}\right)_{\lambda}=1$ and $\left(x . v_{1}, v_{2}\right)_{\lambda}=\left(v_{1}, \varphi(x) . v_{2}\right)_{\lambda}$ for $v_{1}, v_{2} \in V_{\mathbb{C}}(\lambda)$ and $x \in \mathfrak{g}$. It is known [KP83, Theorem 1] that $\Phi$ is an isomorphism of bimodules, called the Peter-Weyl isomorphism for symmetrizable Kac-Moody Lie algebras.

The multiplications $\mathbf{U}\left(\mathfrak{p}_{i}^{-}\right) \otimes \mathbf{U}(\mathfrak{g}) \rightarrow \mathbf{U}(\mathfrak{g})$ and $\mathbf{U}(\mathfrak{g}) \otimes \mathbf{U}\left(\mathfrak{p}_{i}^{+}\right) \rightarrow \mathbf{U}(\mathfrak{g})$ induce coaction morphisms $\mathbf{R}_{\mathbb{C}}(\mathfrak{g}) \rightarrow \mathbb{C}\left[\boldsymbol{P}_{i}^{-}\right] \otimes \mathbf{R}_{\mathbb{C}}(\mathfrak{g})$ and $\mathbf{R}_{\mathbb{C}}(\mathfrak{g}) \rightarrow \mathbf{R}_{\mathbb{C}}(\mathfrak{g}) \otimes \mathbb{C}\left[\boldsymbol{P}_{i}^{+}\right]$. Hence we have the morphisms of schemes $\boldsymbol{P}_{i}^{-} \times \boldsymbol{G}_{\infty} \rightarrow \boldsymbol{G}_{\infty}$ and $\boldsymbol{G}_{\infty} \times \boldsymbol{P}_{i}^{+} \rightarrow \boldsymbol{G}_{\infty}$ which give rise to the left action of $\boldsymbol{P}_{i}^{-}$and the right action of $\boldsymbol{P}_{i}^{+}$on $\boldsymbol{G}_{\infty}$. The scheme $\boldsymbol{G}_{\infty}$ contains a canonical point $e$.

Definition 2.4. Let $G$ be the open subset of $\boldsymbol{G}_{\infty}$ which is given by the union of subsets $\boldsymbol{P}_{i_{1}}^{-} \cdots \boldsymbol{P}_{i_{m}}^{-} e \boldsymbol{P}_{j_{1}}^{+} \cdots \boldsymbol{P}_{j_{n}}^{+} \subset \boldsymbol{G}_{\infty}$, that is,

$$
\boldsymbol{G}=\bigcup_{i_{1}, \cdots, i_{m}, j_{1}, \cdots, j_{n} \in I} \boldsymbol{P}_{i_{1}}^{-} \cdots \boldsymbol{P}_{i_{m}}^{-} e \boldsymbol{P}_{j_{1}}^{+} \cdots \boldsymbol{P}_{j_{n}}^{+} .
$$

Proposition 2.5. (1) The left $\boldsymbol{P}_{i}^{-}$action on $\boldsymbol{G}$ is free and the right $\boldsymbol{P}_{i}^{+}$action on $\boldsymbol{G}$ is free.

(2) The restricted left $\boldsymbol{B}_{-}$actions from the left action of $\boldsymbol{P}_{i}^{-}$on $\boldsymbol{G}$ and the restricted right $\boldsymbol{B}_{+}$action on $\boldsymbol{G}$ from the right action of $\boldsymbol{P}_{i}^{+}$on $\boldsymbol{G}$ are independent of $i \in I$.

(3) For $i \in I$ and $g \in G_{i}$, we have ge $=e g$, where the left action of $G_{i}$ and the right left action of $G_{i}$ are defined via the left action of $\boldsymbol{P}_{i}^{-}$and right action of $\boldsymbol{P}_{i}^{+}$using the decomposition $\boldsymbol{P}_{i}^{ \pm}=G_{i} \ltimes \boldsymbol{N}_{i}^{ \pm}$.

Let $\boldsymbol{N}_{-} \times H \times \boldsymbol{N}_{+} \rightarrow \boldsymbol{G}$ be the open immersion defined by the "multiplication" $(x, y, z) \mapsto$ $x y z$ and denote its image by $\boldsymbol{G}_{0}$. Let

$$
[]_{-} \times[]_{0} \times[]_{+}: \boldsymbol{G}_{0} \rightarrow \boldsymbol{N}_{-} \times H \times \boldsymbol{N}_{+}
$$

be the inverse morphism of the "multiplication". We note that we use only the left $\boldsymbol{B}_{-}$-action and the right $\boldsymbol{B}_{+}$-action on $\boldsymbol{G}$. For the minimal Kac-Moody group $G^{\mathrm{min}}$, it is known the same result holds, see Geiß-Leclerc-Schröer [GLS11, Proposition 7.1].

For the Lie algebra anti-involution $\varphi: \mathfrak{g} \rightarrow \mathfrak{g}$, let $\varphi: \mathbf{U}(\mathfrak{g}) \rightarrow \mathbf{U}(\mathfrak{g})$ be the induced antiinvolution as a $\mathbb{C}$-algebra. We note that $\varphi$ induces the anti-isomorphism of group schemes $\boldsymbol{P}_{i}^{ \pm} \stackrel{\sim}{\rightarrow} \boldsymbol{P}_{i}^{\mp}$ for $i \in I$ and we have the following commutative diagram

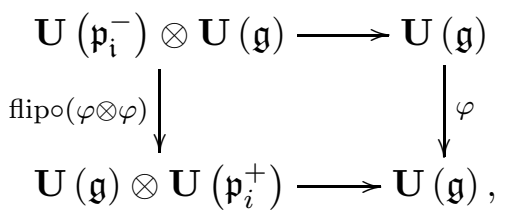

where the horizontal homomorphisms are multiplications. Let ()$^{T}: \boldsymbol{G}_{\infty} \rightarrow \boldsymbol{G}_{\infty}$ be the induced morphism of schemes which intertwines the left $\boldsymbol{P}_{i}^{-}$-action into the right $\boldsymbol{P}_{i}^{+}$-action and vice versa. It is clear that ( $)^{T}$ preserves $\boldsymbol{G}$ and $\boldsymbol{G}_{0}$ by its construction. We denote by ()$^{T}$ the restriction of ()$^{T}$ to $\boldsymbol{G}$ and $\boldsymbol{G}_{0}$ by abuse of notation. For each real root $\alpha \in \Delta_{+}$, ()$^{T}$ maps $\boldsymbol{N}_{+}(\{\alpha\})$ to $\boldsymbol{N}_{-}(\{-\alpha\})$, so ()$^{T}$ induces an involutive map on $G^{\text {min }}$. 
2.5. Schubert cells and Schubert varieties. For a Weyl group element $w \in W$, we specify two lifts $\bar{w}, \overline{\bar{w}} \in G$ of $w \in W$. It is known that $\left\{\overline{s_{i}}\right\}_{i \in I}$ and $\left\{\overline{\overline{s_{i}}}\right\}_{i \in I}$ satisfy braid relations. It follows that the lifts $\bar{w}$ and $\overline{\bar{w}}$ can be uniquely defined by the condition

$$
\begin{aligned}
& \overline{w^{\prime} w^{\prime \prime}}=\overline{w^{\prime}} \cdot \overline{w^{\prime \prime}}, \\
& \overline{\overline{w^{\prime} w^{\prime \prime}}}=\overline{\overline{w^{\prime}}} \cdot \overline{\overline{w^{\prime \prime}}}
\end{aligned}
$$

for $w^{\prime}, w^{\prime \prime} \in W$ with $\ell\left(w^{\prime} w^{\prime \prime}\right)=\ell\left(w^{\prime}\right)+\ell\left(w^{\prime \prime}\right)$.

Proposition 2.6 ([FZ99, Proposition 2.1]). For $w \in W$, we have the following properties:

$$
\begin{aligned}
& \bar{w}^{-1}=\bar{w}^{T}=\overline{\overline{w^{-1}}}, \\
& {\overline{w^{-1}}}^{=} \overline{\bar{w}}^{T}=\overline{\bar{w}}^{-1} .
\end{aligned}
$$

Definition 2.7. The flag scheme $\boldsymbol{X}$ is defined as a quotient scheme $\boldsymbol{G} / \boldsymbol{B}_{+}$.

It is known that $\boldsymbol{X}$ is an essentially smooth and separated (in general, not quasi-compact) scheme over $\mathbb{C}$. Let $e_{\boldsymbol{X}}=\boldsymbol{B}_{+} / \boldsymbol{B}_{+} \in \boldsymbol{X}$ be the image of $e \in \boldsymbol{G}$.

Notation 2.8. For a set $Y$ with a left (resp. right) $H$-action and $w \in W$, we write $\bar{w} Y$ as $w Y$ (resp. $Y \bar{w}$ as $Y w$ ).

Definition 2.9. (1) For $w \in W$, we set $\stackrel{\circ}{X}_{w}=w N_{-}\left(w^{-1}\right) e_{X} \subset \boldsymbol{X}$ to be the locally closed subscheme of $\boldsymbol{X}$. Let $X_{w}$ be the Zariski closure of $\stackrel{\circ}{X}_{w}$ endowed with the reduced scheme structure. $X_{w}$ (resp. $X_{w}$ ) are called (finite) Schubert varieties (resp. cells).

(2) For $w \in W$, we set $\dot{\boldsymbol{X}}^{w}:=\boldsymbol{B}_{-} \bar{w} e_{\boldsymbol{X}}=\boldsymbol{N}_{-} \bar{w} e_{\boldsymbol{X}} \subset \boldsymbol{X}$ to be the locally closed subscheme of $\boldsymbol{X}$. Let $\boldsymbol{X}^{w}$ be the Zariski closure of $\boldsymbol{X}^{w}$ endowed with the reduced scheme structure. $\boldsymbol{X}^{w}\left(\right.$ resp. $\left.\dot{\boldsymbol{X}}^{w}\right)$ are called cofinite Schubert schemes (resp. cells).

(2) For $w \in W$, we set $\boldsymbol{U}_{w}:=w \boldsymbol{B}_{-} e_{\boldsymbol{X}}=w \boldsymbol{N}_{-} e_{\boldsymbol{X}} \subset \boldsymbol{X}$.

Proposition 2.10 ([KT95, Proposition 1.3.2]). (1) $X_{w}$ is the smallest subscheme of $\boldsymbol{X}$ that is invariant by $G_{i}^{+}$'s and contains $w e_{X}$.

(2) There is an isomorphism

$$
N_{+}(w) \rightarrow \stackrel{\circ}{X}_{w}
$$

given by $x \mapsto x w e_{X}$. In particular $\stackrel{\circ}{X}_{w}$ is isomorphic to the affine space $\mathbb{A}^{\ell(w)}$.

(3) We have $X_{w}=\bigsqcup_{y \leq w} \dot{\circ}_{y}$, where $\leq$ is the Bruhat order on $W$.

We note that the morphism $N_{-}(w) \times \boldsymbol{B}_{+} \rightarrow \boldsymbol{B}_{+} \bar{w} \boldsymbol{B}_{+}$given by $(x, y) \mapsto x^{T} \bar{w} y$ is an isomorphism.

Remark 2.11. We note that the union $X:=\bigcup_{w \in W} X_{w} \subset \boldsymbol{X}$ has a structure of an ind-scheme over $\mathbb{C}$ and it is also called the flag variety. We have isomorphisms $G^{\mathrm{min}} / B_{+} \stackrel{\sim}{\rightarrow} G / \boldsymbol{B}_{+}=X$, see [Kum02, 7.4.5 Proposition].

Proposition 2.12 ([KT95, Proposition 1.3.1]). (1) There is an isomorphism

$$
\boldsymbol{N}_{-}^{\prime}(w) \rightarrow \dot{\circ}^{w}
$$

given by $x \mapsto x \bar{w} e_{\boldsymbol{X}}$. In particular, $\dot{\boldsymbol{X}}^{w}$ is affine space with $\operatorname{codim} \boldsymbol{X}^{w}=\ell(w)$.

(2) We have $\boldsymbol{X}=\bigsqcup_{w \in W} \stackrel{\circ}{ }^{w}$.

(3) We have $\boldsymbol{X}^{w}=\bigsqcup_{y \geq w} \stackrel{\circ}{\boldsymbol{X}}^{y}$ for $w \in W$. 
Corollary 2.13 (Birkhoff decomposition). We have $\boldsymbol{G}=\bigsqcup_{w \in W} \boldsymbol{B}_{-} \bar{w} \boldsymbol{B}_{+}$.

Proposition 2.14 ([Kas89]). $\boldsymbol{U}_{w}$ is an affine open subset of $\boldsymbol{X}$ and there is an isomorphism

$$
\left(\boldsymbol{N}_{-}^{\prime}(w)\right) \times\left(N_{+}(w)\right) \stackrel{\sim}{\rightarrow} \boldsymbol{U}_{w}
$$

which is given by $(x, y) \mapsto x y w e_{X}$. In particular, $\boldsymbol{N}_{-} \rightarrow \boldsymbol{X}$ given by $n_{-} \mapsto n_{-} e_{\boldsymbol{X}}$ is an open immersion.

2.6. Unipotent cells and their automorphisms. In this subsection, we consider $\boldsymbol{N}_{-}$as an open subscheme of $\boldsymbol{X}$ via the open immersion in Proposition 2.14.

Definition 2.15. For $w \in W$, we set

$$
N_{-}^{w}:=N_{-} \cap \stackrel{\circ}{X}_{w} \subset \boldsymbol{X},
$$

$N_{-}^{w}$ is called the unipotent cell.

Since $w N_{-}\left(w^{-1}\right) \boldsymbol{B}_{+} \subset G$, we have

$$
\begin{aligned}
w N_{-}\left(w^{-1}\right) \boldsymbol{B}_{+} \cap \boldsymbol{N}_{-} \boldsymbol{B}_{+} & =w N_{-}\left(w^{-1}\right) \boldsymbol{B}_{+} \cap \boldsymbol{N}_{-} \boldsymbol{B}_{+} \cap G \\
& =w N_{-}\left(w^{-1}\right) \boldsymbol{B}_{+} \cap N_{-} \boldsymbol{B}_{+} .
\end{aligned}
$$

Therefore we have $\boldsymbol{N}_{-} \cap \stackrel{\circ}{X}_{w}=N_{-} \cap \stackrel{\circ}{X}_{w}$. Similarly, it can be shown that $\boldsymbol{N}_{-} \cap X_{w}=N_{-} \cap X_{w}$. Since $\boldsymbol{N}_{-} \subset \boldsymbol{X}$ is a Zariski open immersion, $N_{-} \cap X_{w}=\boldsymbol{N}_{-} \cap X_{w} \subset X_{w}$ is an open immersion and $N_{-} \cap X_{w}$ is a closed (affine) subscheme of $\boldsymbol{N}_{-}$. Moreover $N_{-} \cap X_{w}$ is reduced. It also coincides with the scheme-theoretic intersection.

We shall describe the coordinate ring of $N_{-} \cap X_{w}$ explicitly, that is, we describe the kernel of the quotient map $\mathbb{C}\left[\boldsymbol{N}_{-}\right] \rightarrow \mathbb{C}\left[N_{-} \cap X_{w}\right]$, after preparing some notations. For a reduced expression $\boldsymbol{i}=\left(i_{1}, \cdots, i_{\ell}\right) \in I(w)$ of a Weyl group element $w \in W$, we consider a morphism $y_{i}: \mathbb{A}^{\ell(w)} \rightarrow N_{-}$defined by

$$
y_{\boldsymbol{i}}\left(z_{1}, \cdots, z_{\ell}\right):=\exp \left(z_{1} f_{i_{1}}\right) \cdots \exp \left(z_{\ell} f_{i_{\ell}}\right) .
$$

We note that the associated ring homomorphism $y_{i}^{*}: \mathbb{C}\left[\boldsymbol{N}_{-}\right] \rightarrow \mathbb{C}\left[\mathbb{A}^{\ell(w)}\right]=\mathbb{C}\left[z_{1}, \ldots, z_{\ell}\right]$ is nothing but the (classical) Feigin map or Geiß-Leclerc-Schröer's $\varphi$-map (see Geiß-LeclercSchröer [GLS11, Section 6]). Moreover, set

$$
\mathbf{U}_{w}^{-}:=\sum_{a_{1}, \cdots, a_{\ell} \in \mathbb{Z}_{\geq 0}} \mathbb{C} f_{i_{1}}^{a_{1}} \cdots f_{i_{\ell}}^{a_{\ell}} \subset \mathbf{U}\left(\mathfrak{n}_{-}\right) .
$$

Then $\mathbf{U}_{w}^{-}$is independent of the choice of $\boldsymbol{i} \in I(w)$ (see also Proposition 3.34 and Remark 3.35).

Proposition 2.16. For $w \in W$, we have isomorphisms of $\mathbb{C}$-algebras:

$$
\mathbb{C}\left[\boldsymbol{N}_{-}\right] /\left(\mathbf{U}_{w}^{-}\right)^{\perp} \stackrel{\sim}{\longrightarrow} \mathbb{C}\left[N_{-} \cap X_{w}\right],
$$

here $\left(\mathbf{U}_{w}^{-}\right)^{\perp}:=\left\{f \in \mathbf{U}\left(\mathfrak{n}_{-}\right)_{\mathrm{gr}}^{*} \mid f\left(\mathbf{U}_{w}^{-}\right)=0\right\}$ (recall that $\mathbb{C}\left[\boldsymbol{N}_{-}\right]=\mathbf{U}\left(\mathfrak{n}_{-}\right)_{\mathrm{gr}}^{*}$ ).

Proof. Let us consider the morphism $y_{i}: \mathbb{A}^{\ell(w)} \rightarrow \boldsymbol{N}_{-}$. It can be shown that the set-theoretic image of the morphism $y_{i}$ is included in $N_{-} \cap X_{w}$ and the set-theoretic image of $\left.y_{\boldsymbol{i}}\right|_{\mathbb{G}_{m}^{\ell(w)}}$ is dense in $N_{-} \cap \stackrel{\circ}{X}_{w}$ (cf. Kum02, Proposition 7.1.15]). Since $\mathbb{A}^{\ell(w)}$ is reduced and the Zariski closure of $N_{-} \cap \dot{\circ}_{w}$ is $N_{-} \cap X_{w}$, the scheme-theoretic image of $y_{i}$ (into $N_{-}$) is $N_{-} \cap X_{w}$. 
The claim follows from the claim $\left(\mathbf{U}_{w}^{-}\right)^{\perp}=\left\{f \in \mathbb{C}\left[\boldsymbol{N}_{-}\right] \mid y_{\boldsymbol{i}}^{*}(f)=0\right\}$ which is clear from the definition of Geiß-Leclerc-Schröer's $\varphi$-map.

We next describe the coordinate rings of $\mathbb{C}\left[N_{-}^{w}\right]$ and $\mathbb{C}\left[N_{-}(w) \cap w G_{0}^{\min }\right]$. For $\lambda \in P_{+}$, we set $u_{w \lambda}:=\bar{w} u_{\lambda}$. We set $\Delta_{w \lambda, \lambda}:=\Phi_{\lambda}\left(u_{w \lambda}^{\mathrm{r}} \otimes u_{\lambda}\right) \in R_{\mathbb{C}}(\mathfrak{g})$. We regard $\Delta_{w \lambda, \lambda}$ as a regular function on $\boldsymbol{G}_{\infty}$ and its restriction to $\boldsymbol{G}$. We have the following recognizing criterion of the point in the Schubert cells in the Schubert variety in terms of (unipotent) minors $\Delta_{w \lambda, \lambda}$. It is proved by Williams [Wil13, Lemma 4.15] in the "minimal" Kac-Moody group setting.

Lemma 2.17. For $g \in G^{\mathrm{min}}$ and a point $g e_{X}$ on the Schubert variety $X_{w}$ belongs to the Schubert cell $X_{w}$ if and only if $\Delta_{w \lambda, \lambda}\left(g e_{X}\right) \neq 0$ for $\lambda \in P_{+}$, where $e_{X}:=B_{+} / B_{+} \in G^{\mathrm{min}} / B_{+}$.

Since $N_{-}^{w}$ is also reduced, the set-theoretic intersection $N_{-} \cap \stackrel{\circ}{X}_{w}$ coincides with the schemetheoretic intersection, we obtain the following corollary.

Corollary 2.18. For $w \in W$, we have isomorphisms of $\mathbb{C}$-algebras:

$$
\left(\mathbb{C}\left[\boldsymbol{N}_{-}\right] /\left(\mathbf{U}_{w}^{-}\right)^{\perp}\right)\left[\left[D_{w \lambda, \lambda}^{\mathbb{C}}\right]_{w}^{-1} \mid \lambda \in P_{+}\right] \stackrel{\sim}{\rightarrow} \mathbb{C}\left[N_{-}^{w}\right]
$$

where $\left[D_{w \lambda, \lambda}^{\mathbb{C}}\right]_{w}=\left.\Delta_{w \lambda, \lambda}\right|_{N_{-} \cap X_{w}}: N_{-} \cap X_{w} \rightarrow \mathbb{C}$ is the restriction of $\Delta_{w \lambda, \lambda}$ to $N_{-} \cap X_{w}$.

By Corollary 2.13, we have

$$
w \boldsymbol{G}_{0}=\left\{g \in \boldsymbol{G} \mid \Delta_{w \lambda, \lambda}(g) \neq 0\right\} .
$$

By [GLS11, Proposition 7.3] we have $G_{0}^{\min }=\boldsymbol{G}_{0} \cap G^{\mathrm{min}}$, in particular, we obtain $N_{-}(w) \cap$ $w G_{0}^{\min }=N_{-}(w) \cap w \boldsymbol{G}_{0}$. Hence we have

$$
\mathbb{C}\left[N_{-}(w)\right]\left[\left(D_{w \lambda, \lambda}^{\mathbb{C}}\right)^{-1} \mid \lambda \in P_{+}\right] \stackrel{\sim}{\rightarrow} \mathbb{C}\left[N_{-}(w) \cap w G_{0}^{\min }\right],
$$

here $D_{w \lambda, \lambda}^{\mathbb{C}}:=\left.\Delta_{w \lambda, \lambda}\right|_{N_{-}(w)}$.

Our next goal is to show Corollary 2.22. This is a classical counterpart of the De ConciniProcesi isomorphisms, which we prove in subsection 4.3. We first recall the (classical) twist isomorphism $\gamma_{w}$ and the (classical) twist automorphism $\eta_{w}$ following Berenstein-Zelevinsky and Geiß-Leclerc-Schröer.

Definition 2.19. For $w \in W$, let $\boldsymbol{O}_{w}:=\boldsymbol{N}_{-} \cap w \boldsymbol{G}_{0}$. We define a map $\tilde{\boldsymbol{\gamma}}_{w}: \boldsymbol{O}_{w} \rightarrow \boldsymbol{N}_{-}$by

$$
\tilde{\gamma}_{w}(z)=\left[z^{T} \bar{w}\right]_{-} .
$$

The following is proved by Berenstein-Zelevinsky [BZ97] (see also Geiß-Leclerc-Schröer GLS11, Proposition 8.4, Proposition 8.5]).

Proposition 2.20. The following properties hold:

(1) The map $\tilde{\boldsymbol{\gamma}}_{w}: \boldsymbol{O}_{w} \rightarrow \boldsymbol{N}_{-}$is a morphism between schemes.

(2) The image of $\tilde{\gamma}_{w}$ is $N_{-}^{w}$.

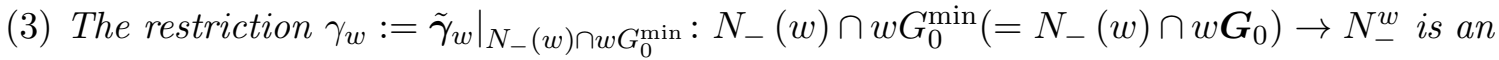
isomorphism.

(4) We have $N_{-}^{w} \subset w G_{0}^{\min }\left(\subset w \boldsymbol{G}_{0}\right)$ and $\eta_{w}:=\left.\tilde{\boldsymbol{\gamma}}_{w}\right|_{N_{-}^{w}}: N_{-}^{w} \rightarrow N_{-}^{w}$ is an automorphism.

(5) Let $\pi_{w}: \boldsymbol{N}_{-} \rightarrow N_{-}(w)$ be the projection for the isomorphism $\boldsymbol{N}_{-}^{\prime}(w) \times N_{-}(w) \stackrel{\sim}{\rightarrow} \boldsymbol{N}_{-}$ given by multiplication (see subsection [2.2). Then $\pi_{w}$ restricts to $N_{-}^{w} \rightarrow N_{-}(w) \cap w G_{0}^{\min }$, and $\eta_{w}=\left.\gamma_{w} \circ \pi_{w}\right|_{N_{-}^{w}}$. 
Remark 2.21. In [GLS11], they define a twist isomorphism and and a twist automorphism as restrictions of the morphism $\tilde{\gamma}_{w}: N_{-} \cap w G_{0}^{\min } \rightarrow N_{-}, z \mapsto\left[z^{T} \bar{w}\right]_{-}$between ind-schemes. Eventually, it turns out that this twist isomorphism (resp. twist automorphism) coincides with our $\gamma_{w}$ (resp. $\left.\eta_{w}\right)$.

Let $\pi_{w}^{*}: \mathbb{C}\left[N_{-}(w)\right] \hookrightarrow \mathbb{C}\left[\boldsymbol{N}_{-}\right]$be the $\mathbb{C}$-algebra homomorphism induced from $\pi_{w}$. Then, by GLS11, Proposition 8.2], the image of $\pi_{w}^{*}$ consists of the left $\boldsymbol{N}_{-}^{\prime}(w)$-invariant functions in $\mathbb{C}\left[\boldsymbol{N}_{-}\right]$. Note that our convention is the transpose of Geiß-Leclerc-Schörer's convention. Moreover, by the calculation in [GLS11, subsection 8.2], a function $\left.\Phi_{\lambda}\left(u_{w \lambda}^{\mathrm{r}} \otimes u\right)\right|_{N_{-}}$is left $N_{-}^{\prime}(w)$-invariant for all $u \in V(\lambda), \lambda \in P_{+}$. Hence

$$
\pi_{w}^{*}\left(\left.\Phi_{\lambda}\left(u_{w \lambda}^{\mathrm{r}} \otimes u\right)\right|_{N_{-}(w)}\right)=\left.\Phi_{\lambda}\left(u_{w \lambda}^{\mathrm{r}} \otimes u\right)\right|_{N_{-}} .
$$

Corollary 2.22. For $w \in W$, we have an isomorphism of $\mathbb{C}$-algebras:

$$
\mathbb{C}\left[N_{-}(w) \cap w G_{0}^{\min }\right] \stackrel{\sim}{\rightarrow} \mathbb{C}\left[N_{-}^{w}\right],
$$

which is induced by localizing the homomorphism $\mathbb{C}\left[N_{-}(w)\right] \stackrel{\pi_{w}^{*}}{\longrightarrow} \mathbb{C}\left[\boldsymbol{N}_{-}\right] \rightarrow \mathbb{C}\left[N_{-} \cap X_{w}\right]$ with respect to $\left\{D_{w \lambda, \lambda}^{\mathbb{C}} \mid \lambda \in P_{+}\right\}$.

Proof. By definition, the composite map $\iota: \mathbb{C}\left[N_{-}(w)\right] \stackrel{\pi_{w}^{*}}{\rightarrow} \mathbb{C}\left[\boldsymbol{N}_{-}\right] \rightarrow \mathbb{C}\left[N_{-} \cap X_{w}\right]$ is induced from the morphism of schemes $\left.\pi_{w}\right|_{N_{-} \cap X_{w}}: N_{-} \cap X_{w} \rightarrow N_{-}(w)$. Moreover, by Corollary 2.18 and (2.2) , the inclusions $N_{-}^{w} \rightarrow N_{-} \cap X_{w}$ and $N_{-}(w) \cap w G_{0}^{\text {min }} \rightarrow N_{-}(w)$ corresponds to the canonical $\mathbb{C}$-algebra homomorphisms

$$
\begin{aligned}
& \iota_{1}: \mathbb{C}\left[N_{-} \cap X_{w}\right] \rightarrow \mathbb{C}\left[N_{-} \cap X_{w}\right]\left[\left[D_{w \lambda, \lambda}^{\mathbb{C}}\right]_{w}^{-1} \mid \lambda \in P_{+}\right] \simeq \mathbb{C}\left[N_{-}^{w}\right], \\
& \iota_{2}: \mathbb{C}\left[N_{-}(w)\right] \rightarrow \mathbb{C}\left[N_{-}(w)\right]\left[\left(D_{w \lambda, \lambda}^{\mathbb{C}}\right)^{-1} \mid \lambda \in P_{+}\right] \simeq \mathbb{C}\left[N_{-}(w) \cap w G_{0}^{\min }\right] .
\end{aligned}
$$

Therefore the composite map $\iota_{1} \circ \iota: \mathbb{C}\left[N_{-}(w)\right] \rightarrow \mathbb{C}\left[N_{-}^{w}\right]$ is induced from $\left.\pi_{w}\right|_{N_{-}^{w}}: N_{-}^{w} \rightarrow$ $N_{-}(w)$. Moreover, by (2.3) $),\left(\iota_{1} \circ \iota\right)\left(D_{w \lambda, \lambda}^{\mathbb{C}}\right)=\left[D_{w \lambda, \lambda}^{\mathbb{C}}\right]_{w}$ for all $u \in V(\lambda), \lambda \in P_{+}$. Hence, by the universality of localization, $\iota_{1} \circ \iota$ extends to $\mathbb{C}\left[N_{-}(w) \cap w G_{0}^{\min }\right] \rightarrow \mathbb{C}\left[N_{-}^{w}\right]$. By construction this is induced from $\left.\pi_{w}\right|_{N_{-}^{w}}: N_{-}^{w} \rightarrow N_{-}(w) \cap w G_{0}^{\text {min }}$, which is an isomorphism of schemes by Proposition 2.20 , Hence we obtain the desired isomorphism $\mathbb{C}\left[N_{-}(w) \cap w G_{0}^{\min }\right] \rightarrow$ $\mathbb{C}\left[N_{-}^{w}\right]$.

We conclude this subsection by describing the classical twist isomorphism $\gamma_{w}$ in terms of matrix coefficients.

Proposition 2.23. Let $\gamma_{w}^{*}: \mathbb{C}\left[N_{-}^{w}\right] \rightarrow \mathbb{C}\left[N_{-}(w) \cap w G_{0}^{\min }\right]$ be the isomorphism of $\mathbb{C}$-algebras induced from $\gamma_{w}$. Then, for $w \in W, \lambda \in P_{+}$and $u \in V(\lambda)$, we have

$$
\gamma_{w}^{*}\left(\left[D_{u, u_{\lambda}}^{\mathbb{C}}\right]_{w}\right)=\frac{D_{u_{w \lambda}, u}^{\mathbb{C}}}{D_{u_{w \lambda}, u_{\lambda}}^{\mathbb{C}}},
$$

here $\left[D_{u, u_{\lambda}}^{\mathbb{C}}\right]_{w}:=\left.\Phi_{\lambda}\left(u^{\mathrm{r}} \otimes u_{\lambda}\right)\right|_{N_{-}^{w}}$ and $D_{u_{w \lambda}, u}^{\mathbb{C}}:=\left.\Phi_{\lambda}\left(u_{w \lambda}^{\mathrm{r}} \otimes u\right)\right|_{N_{-}(w) \cap w G_{0}^{\min }}$ (cf. Corollary 2.18, (2.2) ). 
Proof. We compute the value of functions. For $z \in N_{-}(w) \cap O_{w}$, we have

$$
\begin{aligned}
\left\langle\gamma_{w}^{*}\left(D_{u, u_{\lambda}}^{\mathbb{C}}\right), z\right\rangle & =\left(u, \gamma_{w}(z) u_{\lambda}\right)_{\lambda}=\left(u,\left[z^{T} \bar{w}\right]_{-} u_{\lambda}\right)_{\lambda} \\
& =\left(u, z^{T} \bar{w} u_{\lambda}\right)_{\lambda} /\left(u_{\lambda},\left[z^{T} \bar{w}\right]_{0} u_{\lambda}\right)_{\lambda} \\
& =\left(u, z^{T} \bar{w} u_{\lambda}\right)_{\lambda} /\left(u_{\lambda}, z^{T} \bar{w} u_{\lambda}\right)_{\lambda} \\
& =\left(z u, u_{w \lambda}\right)_{\lambda} /\left(z u_{\lambda}, u_{w \lambda}\right)_{\lambda} .
\end{aligned}
$$

Hence we obtained the claim.

\section{Preliminaries (2) : Quantized enveloping algebras and Canonical bases}

3.1. Quantized enveloping algebras. In this subsection, we present the definitions of quantized enveloping algebras. Let $q$ be an indeterminate.

Notation 3.1. Set

$$
\begin{aligned}
& q_{i}:=q^{\frac{\left(\alpha_{i}, \alpha_{i}\right)}{2}},[n]:=\frac{q^{n}-q^{-n}}{q-q^{-1}} \text { for } n \in \mathbb{Z}, \\
& {\left[\begin{array}{c}
n \\
k
\end{array}\right]:= \begin{cases}\frac{[n][n-1] \cdots[n-k+1]}{[k][k-1] \cdots[1]} & \text { if } n \in \mathbb{Z}, k \in \mathbb{Z}_{>0}, \\
1 & \text { if } n \in \mathbb{Z}, k=0,\end{cases} } \\
& {[n] !:=[n][n-1] \cdots[1] \text { for } n \in \mathbb{Z}>0,[0] !:=1 .}
\end{aligned}
$$

For a rational function $R \in \mathbb{Q}(q)$, we define $R_{i}$ as the rational function obtained from $R$ by substituting $q$ by $q_{i}(i \in I)$.

Definition 3.2. The quantized enveloping algebra $\mathbf{U}_{q}$ associated with a root datum $\left(I, \mathfrak{h}, P,\left\{\alpha_{i}\right\}_{i \in I},\left\{h_{i}\right\}_{i \in I},(\right.$, is the unital associative $\mathbb{Q}(q)$-algebra defined by the generators

$$
e_{i}, f_{i}(i \in I), q^{h}\left(h \in P^{*}\right),
$$

and the relations (i)-(iv) below:

(i) $q^{0}=1, q^{h} q^{h^{\prime}}=q^{h+h^{\prime}}$ for $h, h^{\prime} \in P^{*}$,

(ii) $q^{h} e_{i}=q^{\left\langle h, \alpha_{i}\right\rangle} e_{i} q^{h}, q^{h} f_{i}=q^{-\left\langle h, \alpha_{i}\right\rangle} f_{i} q^{h}$ for $h \in P^{*}, i \in I$,

(iii) $\left[e_{i}, f_{j}\right]=\delta_{i j} \frac{t_{i}-t_{i}^{-1}}{q_{i}-q_{i}^{-1}}$ for $i, j \in I$ where $t_{i}:=q^{\frac{\left(\alpha_{i}, \alpha_{i}\right)}{2} h_{i}}$,

(iv) $\sum_{k=0}^{1-a_{i j}}(-1)^{k}\left[\begin{array}{c}1-a_{i j} \\ k\end{array}\right]_{i} x_{i}^{k} x_{j} x_{i}^{1-a_{i j}-k}=0$ for $i, j \in I$ with $i \neq j$, and $x=e, f$.

The $\mathbb{Q}(q)$-subalgebra of $\mathbf{U}_{q}$ generated by $\left\{e_{i}\right\}_{i \in I}$ (resp. $\left\{f_{i}\right\}_{i \in I},\left\{q^{h}\right\}_{h \in P^{*}},\left\{e_{i}, q^{h}\right\}_{i \in I, h \in P^{*}}$, $\left.\left\{f_{i}, q^{h}\right\}_{i \in I, h \in P^{*}}\right)$ will be denoted by $\mathbf{U}_{q}^{+}\left(\operatorname{resp} . \mathbf{U}_{q}^{-}, \mathbf{U}_{q}^{0}, \mathbf{U}_{\bar{q}}^{\geq 0}, \mathbf{U}_{q}^{\leq 0}\right)$.

For $\alpha \in Q$, write $\left(\mathbf{U}_{q}\right)_{\alpha}:=\left\{x \in \mathbf{U}_{q} \mid q^{h} x q^{-h}=q^{\langle h, \alpha\rangle} x\right.$ for all $\left.h \in P^{*}\right\}$. The elements of $\left(\mathbf{U}_{q}\right)_{\alpha}$ are said to be homogeneous. For a homogeneous element $x \in\left(\mathbf{U}_{q}\right)_{\alpha}$, we set wt $x=\alpha$. For any subset $X \subset \mathbf{U}_{q}$ and $\alpha \in Q$, we set $X_{\alpha}:=X \cap\left(\mathbf{U}_{q}\right)_{\alpha}$. 
The algebra $\mathbf{U}_{q}$ has a Hopf algebra structure. In this paper, we take the coproduct $\Delta: \mathbf{U}_{q} \rightarrow \mathbf{U}_{q} \otimes \mathbf{U}_{q}$, the counit $\varepsilon: \mathbf{U}_{q} \rightarrow \mathbb{Q}(q)$ and the antipode $S: \mathbf{U}_{q} \rightarrow \mathbf{U}_{q}$ as follows:

$$
\begin{array}{rlrl}
\Delta\left(e_{i}\right) & =e_{i} \otimes t_{i}^{-1}+1 \otimes e_{i}, & \varepsilon\left(e_{i}\right)=0, & S\left(e_{i}\right)=-e_{i} t_{i}, \\
\Delta\left(f_{i}\right)=f_{i} \otimes 1+t_{i} \otimes f_{i}, & \varepsilon\left(f_{i}\right)=0, & S\left(f_{i}\right)=-t_{i}^{-1} f_{i}, \\
\Delta\left(q^{h}\right)=q^{h} \otimes q^{h}, & \varepsilon\left(q^{h}\right)=1, & S\left(q^{h}\right)=q^{-h} .
\end{array}
$$

Definition 3.3. Let $\vee: \mathbf{U}_{q} \rightarrow \mathbf{U}_{q}$ be the $\mathbb{Q}(q)$-algebra involution defined by

$$
e_{i}^{\vee}=f_{i}, \quad \quad f_{i}^{\vee}=e_{i}, \quad\left(q^{h}\right)^{\vee}=q^{-h} .
$$

Let $^{-}: \mathbb{Q}(q) \rightarrow \mathbb{Q}(q)$ and ${ }^{-}: \mathbf{U}_{q} \rightarrow \mathbf{U}_{q}$ be the $\mathbb{Q}$-algebra involutions defined by

$$
\bar{q}=q^{-1}, \quad \overline{e_{i}}=e_{i}, \quad \overline{f_{i}}=f_{i}, \quad \overline{q^{h}}=q^{-h} .
$$

Let $*, \varphi, \psi: \mathbf{U}_{q} \rightarrow \mathbf{U}_{q}$ be the $\mathbb{Q}(q)$-algebra anti-involutions defined by

$$
\begin{array}{lll}
*\left(e_{i}\right)=e_{i}, & *\left(f_{i}\right)=f_{i}, & *\left(q^{h}\right)=q^{-h}, \\
\varphi\left(e_{i}\right)=f_{i}, & \varphi\left(f_{i}\right)=e_{i}, & \varphi\left(q^{h}\right)=q^{h} \\
\psi\left(e_{i}\right)=q_{i}^{-1} t_{i}^{-1} f_{i}, & \psi\left(f_{i}\right)=q_{i}^{-1} t_{i} e_{i}, & \psi\left(q^{h}\right)=q^{h} .
\end{array}
$$

Remark that $\psi$ is also a $\mathbb{Q}(q)$-coalgebra homomorphism, and $\varphi=\vee \circ *=* \circ \vee$.

In this paper, we also use the following variant $\check{\mathbf{U}}_{q}$ of the quantized enveloping algebra $\mathbf{U}_{q}$.

Definition 3.4. A variant $\check{\mathbf{U}}_{q}$ of the quantized enveloping algebra $\mathbf{U}_{q}$ is the unital associative $\mathbb{Q}(q)$-algebra defined by the generators

$$
e_{i}, f_{i}(i \in I), q^{\mu}(\mu \in P)
$$

and the relations (i)-(iv) below:

(i) $q^{0}=1, q^{\mu} q^{\mu^{\prime}}=q^{\mu+\mu^{\prime}}$ for $\mu, \mu^{\prime} \in P$,

(ii) $q^{\mu} e_{i}=q^{\left(\mu, \alpha_{i}\right)} e_{i} q^{\mu}, q^{\mu} f_{i}=q^{-\left(\mu, \alpha_{i}\right)} f_{i} q^{\mu}$ for $\mu \in P, i \in I$,

(iii) $\left[e_{i}, f_{j}\right]=\delta_{i j} \frac{t_{i}-t_{i}^{-1}}{q_{i}-q_{i}^{-1}}$ for $i, j \in I$ where $t_{i}:=q^{\alpha_{i}}$ (abuse of notation),

(iv) $\sum_{k=0}^{1-a_{i j}}(-1)^{k}\left[\begin{array}{c}1-a_{i j} \\ k\end{array}\right]_{i} x_{i}^{k} x_{j} x_{i}^{1-a_{i j}-k}=0$ for $i, j \in I$ with $i \neq j$, and $x=e, f$.

The $\mathbb{Q}(q)$-algebra $\check{\mathbf{U}}_{q}$ has a Hopf algebra structure given by the same formulae as $\mathbf{U}_{q}$. The notions, notations and maps defined in Definition 3.2 and 3.3 are immediately translated into those for $\check{\mathbf{U}}_{q}$. Note that $\check{\mathbf{U}}_{q}^{ \pm}$can be identified with $\mathbf{U}_{q}^{ \pm}$via $e_{i} \mapsto e_{i}$ and $f_{i} \mapsto f_{i}$, respectively.

3.2. Drinfeld pairings and Lusztig pairings. Some non-degenerate bilinear forms play a role of bridges between quantized enveloping algebras and their dual objects.

Proposition 3.5 ([Dri87, Tan92]). There uniquely exists a $\mathbb{Q}(q)$-bilinear map $(,)_{D}: \check{\mathbf{U}}_{\bar{q}}^{\geq 0} \times$ $\mathbf{U}_{q}^{\leq 0} \rightarrow \mathbb{Q}(q)$ such that

(i) $\left(\Delta(x), y_{1} \otimes y_{2}\right)_{D}=\left(x, y_{1} y_{2}\right)_{D}$ for $x \in \check{\mathbf{U}}_{\bar{q}}^{\geq 0}, y_{1}, y_{2} \in \mathbf{U}_{q}^{\leq 0}$,

(ii) $\left(x_{2} \otimes x_{1}, \Delta(y)\right)_{D}=\left(x_{1} x_{2}, y\right)_{D}$ for $x_{1}, x_{2} \in \check{\mathbf{U}}_{\bar{q}}^{\geq 0}, y \in \mathbf{U}_{\bar{q}}^{\leq 0}$, 
(iii) $\left(e_{i}, q^{h}\right)_{D}=\left(q^{\mu}, f_{i}\right)_{D}=0$ for $i \in I$ and $h \in P^{*}, \mu \in P$,

(iv) $\left(q^{\mu}, q^{h}\right)_{D}=q^{-\langle h, \mu\rangle}$ for $\mu \in P, h \in P^{*}$,

(v) $\left(e_{i}, f_{j}\right)_{D}=-\delta_{i j} \frac{1}{q_{i}-q_{i}^{-1}}$ for $i, j \in I$,

here the $\mathbb{Q}(q)$-bilinear map $(,)_{D}: \check{\mathbf{U}}_{\bar{q}}^{\geq 0} \otimes \check{\mathbf{U}}_{\bar{q}}^{\geq 0} \times \mathbf{U}_{q}^{\leq 0} \otimes \mathbf{U}_{\bar{q}}^{\leq 0} \rightarrow \mathbb{Q}(q)$ is defined by $\left(x_{1} \otimes\right.$ $\left.x_{2}, y_{1} \otimes y_{2}\right)_{D}=\left(x_{1}, y_{1}\right)_{D}\left(x_{2}, y_{2}\right)_{D}$ for $x_{1}, x_{2} \in \check{\mathbf{U}}_{\bar{q}}^{\geq 0}, y_{1}, y_{2} \in \mathbf{U}_{\bar{q}}^{\leq 0}$.

The bilinear map $(,)_{D}$ is called the Drinfeld pairing. It has the following properties:

(1) For $\alpha, \beta \in Q_{+},\left.(,)_{D}\right|_{\left(\check{\mathbf{U}}_{\bar{q}}^{\geq 0}\right)_{\alpha} \times\left(\mathbf{U}_{q}^{\leq 0}\right)_{-\beta}}=0$ unless $\alpha=\beta$.

(2) For $\alpha \in Q_{+},\left.(,)_{D}\right|_{\left(\mathbf{U}_{q}^{+}\right)_{\alpha} \times\left(\mathbf{U}_{q}^{-}\right)_{-\alpha}}$ is non-degenerate.

(3) $\left(q^{\mu} x, q^{h} y\right)_{D}=q^{-\langle h, \mu\rangle}(x, y)_{D}$ for $\mu \in P, h \in P^{*}$ and $x \in \mathbf{U}_{q}^{+}, y \in \mathbf{U}_{q}^{-}$.

Definition 3.6. For $i \in I$, define the $\mathbb{Q}(q)$-linear maps $e_{i}^{\prime}$ and ${ }_{i} e^{\prime}: \mathbf{U}_{q}^{-} \rightarrow \mathbf{U}_{q}^{-}$by

$$
\begin{aligned}
e_{i}^{\prime}(x y) & =e_{i}^{\prime}(x) y+q_{i}^{\left\langle h_{i}, \mathrm{wt} x\right\rangle} x e_{i}^{\prime}(y), & e_{i}^{\prime}\left(f_{j}\right) & =\delta_{i j}, \\
{ }_{i} e^{\prime}(x y) & =q_{i}^{\left\langle h_{i}, \mathrm{wt} y\right\rangle}{ }_{i} e^{\prime}(x) y+x_{i} e^{\prime}(y), & { }_{i} e^{\prime}\left(f_{j}\right) & =\delta_{i j}
\end{aligned}
$$

for homogeneous elements $x, y \in \mathbf{U}_{q}^{-}$. For $i \in I$, define the $\mathbb{Q}(q)$-linear maps $f_{i}^{\prime}$ and ${ }_{i} f^{\prime}: \mathbf{U}_{q}^{+} \rightarrow$ $\mathbf{U}_{q}^{+}$by

$$
\begin{aligned}
f_{i}^{\prime}(x y) & =f_{i}^{\prime}(x) y+q_{i}^{-\left\langle h_{i}, \mathrm{wt} x\right\rangle} x f_{i}^{\prime}(y), & & f_{i}^{\prime}\left(e_{j}\right)=\delta_{i j}, \\
{ }_{i} f^{\prime}(x y) & =q_{i}^{-\left\langle h_{i}, \mathrm{wt} y\right\rangle}{ }_{i} f^{\prime}(x) y+x_{i} f^{\prime}(y), & & { }_{i}^{\prime}\left(e_{j}\right)=\delta_{i j}
\end{aligned}
$$

for homogeneous elements $x, y \in \mathbf{U}_{q}^{+}$.

Definition 3.7. Define the $\mathbb{Q}(q)$-bilinear form $(,)_{L}: \mathbf{U}_{q}^{-} \times \mathbf{U}_{q}^{-} \rightarrow \mathbb{Q}(q)$ by $(x, y)_{L}:=$ $(\psi(x), y)_{D}$ for $x, y \in \mathbf{U}_{q}^{-}$. Note that $x$ is regarded as an element of $\check{\mathbf{U}}_{\bar{q}}^{\leq 0}$, while $y$ is considered as an element of $\mathbf{U}_{\bar{q}}^{\leq 0}$. See Definition 3.4. Then this bilinear form satisfies

$$
(1,1)_{L}=1, \quad\left(f_{i} x, y\right)_{L}=\frac{1}{1-q_{i}^{2}}\left(x, e_{i}^{\prime}(y)\right)_{L}, \quad\left(x f_{i}, y\right)_{L}=\frac{1}{1-q_{i}^{2}}\left(x,{ }_{i} e^{\prime}(y)\right)_{L}
$$

This is a symmetric bilinear form, called the Lusztig pairing. In fact, $(,)_{L}$ is the unique symmetric $\mathbb{Q}(q)$-bilinear form satisfying the properties above. Moreover, $(,)_{L}$ is non-degenerate and has the following property:

$$
(*(x), *(y))_{L}=(x, y)_{L}
$$

for all $x, y \in \mathbf{U}_{q}^{-}$.

Similarly, define the $\mathbb{Q}(q)$-bilinear form $(,)_{L}^{+}: \mathbf{U}_{q}^{+} \times \mathbf{U}_{q}^{+} \rightarrow \mathbb{Q}(q)$ by $(x, y)_{L}^{+}:=(x, \psi(y))_{D}$ for $x, y \in \mathbf{U}_{q}^{+}$. Then this bilinear form satisfies

$$
(1,1)_{L}^{+}=1, \quad\left(e_{i} x, y\right)_{L}^{+}=\frac{1}{1-q_{i}^{2}}\left(x, f_{i}^{\prime}(y)\right)_{L}^{+}, \quad\left(x e_{i}, y\right)_{L}^{+}=\frac{1}{1-q_{i}^{2}}\left(x,{ }_{i} f^{\prime}(y)\right)_{L}^{+} .
$$

The forms $(,)_{L}$ and $(,)_{L}^{+}$are related as follows:

$$
(x, y)_{L}=\left(x^{\vee}, y^{\vee}\right)_{L}^{+}
$$

for all $x, y \in \mathbf{U}_{q}^{-}$. See [Lus10, Chapter 1] for more details. 
The following Lemma can be proved easily from the definition, it is left as an exercise for readers.

Lemma 3.8. For $\mu \in P, h \in P^{*}, y_{1}, y_{2} \in \mathbf{U}_{q}^{-}$and $x_{1}, x_{2} \in \mathbf{U}_{q}^{+}$, we have

$$
\left(\psi\left(y_{1} q^{\mu}\right), y_{2} q^{h}\right)_{D}=q^{-\langle h, \mu\rangle}\left(y_{1}, y_{2}\right)_{L}, \quad\left(x_{1} q^{\mu}, \psi\left(x_{2} q^{h}\right)\right)_{D}=q^{-\langle h, \mu\rangle}\left(x_{1}, x_{2}\right)_{L}^{+} .
$$

Definition 3.9. For a homogeneous $x \in \mathbf{U}_{q}^{-}$, we define $\sigma(x)=\sigma_{L}(x) \in \mathbf{U}_{q}^{-}$by the property that

$$
(\sigma(x), y)_{L}=\overline{(x, \bar{y})_{L}}
$$

for an arbitrary $y \in \mathbf{U}_{q}^{-}$. By the non-degeneracy of $(,)_{L}$, the element $\sigma(x)$ is well-defined. This map $\sigma: \mathbf{U}_{q}^{-} \rightarrow \mathbf{U}_{q}^{-}$is called the dual bar-involution.

Proposition 3.10 ([Kim12, Proposition 3.2], [KO18, Proposition 2.6]). For a homogeneous element $x \in \mathbf{U}_{q}^{-}$, we have

$$
\sigma(x)=(-1)^{\mathrm{ht}(\mathrm{wt} x)} q^{(\mathrm{wt} x, \mathrm{wt} x) / 2-(\operatorname{wt} x, \rho)}(-\circ *)(x) .
$$

In particular, for homogeneous elements $x, y \in \mathbf{U}_{q}^{-}$, we have

$$
\sigma(x y)=q^{(\text {wt } x, \text { wt } y)} \sigma(y) \sigma(x) .
$$

Definition 3.11. Define a $\mathbb{Q}(q)$-linear isomorphism $c_{\mathrm{tw}}: \mathbf{U}_{q}^{-} \rightarrow \mathbf{U}_{q}^{-}$by

$$
x \mapsto q^{(\text {wt } x, \text { wt } x) / 2-(\text { wt } x, \rho)} x
$$

for every homogeneous element $x \in \mathbf{U}_{q}^{-}$. Set $\sigma^{\prime}:=c_{\mathrm{tw}}^{-1} \circ \sigma: \mathbf{U}_{q}^{-} \rightarrow \mathbf{U}_{q}^{-}$. We call $\sigma^{\prime}$ the twisted dual bar involution. By Proposition 3.10, $\sigma^{\prime}(x)=(-1)^{\mathrm{ht}(\mathrm{wt} x)}(-\circ *)(x)$ for every homogeneous element $x \in \mathbf{U}_{q}^{-}$. In particular, $\sigma^{\prime}$ is a $\mathbb{Q}$-algebra anti-involution.

Remark 3.12. Let $x \in \mathbf{U}_{q}^{-}$be a homogeneous element. Then,

$$
\sigma(x)=x \text { if and only if } \sigma^{\prime}(x)=q^{-(\operatorname{wt} x, \operatorname{wt} x) / 2+(\operatorname{wt} x, \rho)} x .
$$

3.3. Canonical/Dual canonical bases. In this subsection, we briefly review the properties of canonical/dual canonical bases of the quantized enveloping algebras and its integrable highest weight modules. See, for example, Kas95 for the fundamental results on crystal bases and canonical bases.

Definition 3.13. For $\lambda \in P_{+}$, denote by $V(\lambda)$ the integrable highest weight $\mathbf{U}_{q}$-module generated by a highest weight vector $u_{\lambda}$ of weight $\lambda$. Define the surjective $\mathbf{U}_{q}^{-}$-module homomorphism $\pi_{\lambda}: \mathbf{U}_{q}^{-} \rightarrow V(\lambda)$ by

$$
\pi_{\lambda}(y)=y \cdot u_{\lambda}
$$

There exists a unique $\mathbb{Q}(q)$-bilinear form $(,)_{\lambda}^{\varphi}: V(\lambda) \times V(\lambda) \rightarrow \mathbb{Q}(q)$ such that

$$
\left(u_{\lambda}, u_{\lambda}\right)_{\lambda}^{\varphi}=1 \quad\left(x . u_{1}, u_{2}\right)_{\lambda}^{\varphi}=\left(u_{1}, \varphi(x) \cdot u_{2}\right)_{\lambda}^{\varphi}
$$

for $u_{1}, u_{2} \in V(\lambda)$ and $x \in \mathbf{U}_{q}$. Then the form $(,)_{\lambda}^{\varphi}$ is non-degenerate and symmetric. See, for example, [GLS13, subsection 2.2, the equality (3.10)].

Set $\mathcal{A}:=\mathbb{Q}\left[q^{ \pm 1}\right]$ and $x_{i}^{(n)}:=x_{i}^{n} /[n]_{i} ! \in \mathbf{U}_{q}$ for $i \in I, n \in \mathbb{Z}_{\geq 0}, x=e, f$. Denote by $\mathbf{U}_{\mathcal{A}}^{-}$the $\mathcal{A}$-subalgebra of $\mathbf{U}_{q}^{-}$generated by the elements $\left\{f_{i}^{(n)}\right\}_{i \in I, n \in \mathbb{Z}_{\geq 0}}$ and we set

$$
\mathbf{A}_{\mathbb{Q}\left[q^{ \pm 1}\right]}\left[\mathbf{N}_{-}\right]:=\left\{x \in \mathbf{U}_{q}^{-} \mid\left(x, \mathbf{U}_{\mathcal{A}}^{-}\right)_{L} \subset \mathcal{A}\right\} .
$$


Lusztig [Lus90a, Lus91, Lus10] and Kashiwara KKas91] have constructed the specific $\mathbb{Q}(q)$ basis $\mathbf{B}^{\text {low }}$ (resp. $\mathbf{B}^{\text {low }}(\lambda), \lambda \in P_{+}$) of $\mathbf{U}_{q}^{-}$(resp. $V(\lambda)$ ), called the canonical basis (or the lower global basis), which is also an $\mathcal{A}$-basis of $\mathbf{U}_{\mathcal{A}}^{-}$(resp. $\left.V(\lambda)_{\mathcal{A}}:=\mathbf{U}_{\mathcal{A}}^{-} u_{\lambda}\right)$. Moreover the elements of $\mathbf{B}^{\text {low }}$ (resp. $\mathbf{B}^{\text {low }}(\lambda)$ ) are parametrized by the Kashiwara crystal $\mathscr{B}(\infty)$ (resp. $\left.\mathscr{B}(\lambda)\right)$. We write

$$
\mathbf{B}^{\text {low }}=\left\{G^{\text {low }}(b) \mid b \in \mathscr{B}(\infty)\right\} \quad \mathbf{B}^{\text {low }}(\lambda)=\left\{G_{\lambda}^{\text {low }}(b) \mid b \in \mathscr{B}(\lambda)\right\} .
$$

We follow the notation in Kas95] concerning the crystal $\left(\mathscr{B}(\infty)\right.$; wt, $\left.\left\{\tilde{e}_{i}\right\}_{i \in I},\left\{\tilde{f}_{i}\right\}_{i \in I},\left\{\varepsilon_{i}\right\}_{i \in I},\left\{\varphi_{i}\right\}_{i \in I}\right)$, $\left(\mathscr{B}(\lambda)\right.$; wt, $\left.\left\{\tilde{e}_{i}\right\}_{i \in I},\left\{\tilde{f}_{i}\right\}_{i \in I},\left\{\varepsilon_{i}\right\}_{i \in I},\left\{\varphi_{i}\right\}_{i \in I}\right)$. The unique element of $\mathscr{B}(\infty)$ with weight 0 is denoted by $u_{\infty}$, and the unique element of $\mathscr{B}(\lambda)$ with weight $w \lambda$ is denoted by $u_{w \lambda}$ for $\lambda \in P_{+}$ and $w \in W$ by abuse of notation.

Denote by $\mathbf{B}^{\text {up }}\left(\operatorname{resp} . \mathbf{B}^{\text {up }}(\lambda)\right)$ the basis of $\mathbf{U}_{q}^{-}(\operatorname{resp} . V(\lambda))$ dual to $\mathbf{B}^{\text {low }}\left(\operatorname{resp} . \mathbf{B}^{\text {low }}(\lambda)\right.$ ) with respect to the bilinear form $(,)_{L}\left(\operatorname{resp} .(,)_{\lambda}^{\varphi}\right)$, that is, $\mathbf{B}^{\text {up }}=\left\{G^{\text {up }}(b)\right\}_{b \in \mathscr{B}(\infty)}\left(\operatorname{resp} \cdot \mathbf{B}^{\text {up }}(\lambda)=\right.$ $\left.\left\{G_{\lambda}^{\mathrm{up}}(b)\right\}_{b \in \mathscr{B}(\lambda)}\right)$ such that

$$
\left(G^{\text {low }}(b), G^{\text {up }}\left(b^{\prime}\right)\right)_{L}=\delta_{b, b^{\prime}} \quad\left(\operatorname{resp} .\left(G_{\lambda}^{\text {low }}(b), G_{\lambda}^{\mathrm{up}}\left(b^{\prime}\right)\right)_{\lambda}^{\varphi}=\delta_{b, b^{\prime}}\right)
$$

for any $b, b^{\prime} \in \mathscr{B}(\infty)$ (resp. $b, b^{\prime} \in \mathscr{B}(\lambda)$ ).

Proposition 3.14 ([Kas91, Theorem 5, Lemma 7.3.2], [Lus10, Theorem 14.4.11]). Let $\lambda \in$ $P_{+}$. There exists a surjective map $\pi_{\lambda}: \mathscr{B}(\infty) \rightarrow \mathscr{B}(\lambda) \coprod\{0\}$ such that

$$
\pi_{\lambda}\left(G^{\text {low }}(b)\right)=G_{\lambda}^{\text {low }}\left(\pi_{\lambda}(b)\right)
$$

for $b \in \mathscr{B}(\infty)$, here we set $G_{\lambda}^{\text {low }}(0):=0$ as a convention. Moreover, $\pi_{\lambda}$ induces a bijection $\pi_{\lambda}^{-1}(\mathscr{B}(\lambda)) \rightarrow \mathscr{B}(\lambda)$.

Definition 3.15. Let $\lambda \in P_{+}$. Define $j_{\lambda}: V(\lambda) \hookrightarrow \mathbf{U}_{q}^{-}$as the dual homomorphism of $\pi_{\lambda}$ given by the non-degenerate bilinear forms $(,)_{\lambda}^{\varphi}: V(\lambda) \times V(\lambda) \rightarrow \mathbb{Q}(q)$ and $(,)_{L}: \mathbf{U}_{q}^{-} \times \mathbf{U}_{q}^{-} \rightarrow$ $\mathbb{Q}(q)$, that is

$$
\left(j_{\lambda}(v), y\right)_{L}=\left(v, \pi_{\lambda}(y)\right)_{\lambda}^{\varphi}\left(=\left(v, y \cdot u_{\lambda}\right)_{\lambda}^{\varphi}\right) .
$$

The following proposition immediately follows from Proposition 3.14 .

Proposition 3.16. There is an injective map $\bar{\jmath}_{\lambda}: \mathscr{B}(\lambda) \hookrightarrow \mathscr{B}(\infty)$ such that

$$
\left(G_{\lambda}^{\text {up }}(b), G^{\text {low }}\left(b^{\prime}\right) \cdot u_{\lambda}\right)_{\lambda}^{\varphi}=\delta_{b^{\prime}, \bar{\jmath}_{\lambda}(b)}
$$

for any $b \in \mathscr{B}(\lambda)$ and $b^{\prime} \in \mathscr{B}(\infty)$. That is, we have $j_{\lambda}\left(G_{\lambda}^{\text {up }}(b)\right)=G^{\text {up }}\left(\bar{\jmath}_{\lambda}(b)\right)$.

Remark 3.17. Let $\lambda \in P_{+}$. Then,

- wt $\bar{\jmath}_{\lambda}(b)=$ wt $b-\lambda$ for $b \in \mathscr{B}(\lambda)$, and

- $\bar{\jmath}_{\lambda}\left(\pi_{\lambda}(b)\right)=b$ for $b \in \pi_{\lambda}^{-1}(\mathscr{B}(\lambda))$.

Proposition 3.18 ([Kas91, Lemma 7.3.4], Lus10, 13.1.11]). For all $b \in \mathscr{B}(\infty)$, we have $\overline{G^{\text {low }}(b)}=G^{\text {low }}(b)$.

Note that Proposition 3.18 immediately implies $\sigma\left(G^{\mathrm{up}}(b)\right)=G^{\mathrm{up}}(b)$ for $b \in \mathscr{B}(\infty)$.

Proposition 3.19 ([Kas93a, Theorem 2.1.1]). There exists an bijection $*: \mathscr{B}(\infty) \rightarrow \mathscr{B}(\infty)$ such that

for $b \in \mathscr{B}(\infty)$.

$$
*\left(G^{\mathrm{low}}(b)\right)=G^{\mathrm{low}}(* b)
$$


Remark that Proposition 3.19 implies $*\left(G^{\mathrm{up}}(b)\right)=G^{\mathrm{up}}(* b)$ for $b \in \mathscr{B}(\infty)$. See the equality (3.1).

Definition 3.20. The bijections $*$ give new crystal structures on $\mathscr{B}(\infty)$, defined by the maps

$$
\mathrm{wt}^{*}:=\mathrm{wt} \circ *=\mathrm{wt}, \varepsilon_{i}^{*}:=\varepsilon \circ *, \varphi_{i}^{*}:=\varphi \circ *, \tilde{e}_{i}^{*}:=* \circ \tilde{e}_{i} \circ *, \tilde{f}_{i}^{*}:=* \circ \tilde{f}_{i} \circ * .
$$

Proposition 3.21 ([Kas91, Theorem 7] [Kas93a, Theorem 2.1.1]). Let $\lambda \in P_{+}$. Then we have

$$
\bar{\jmath}_{\lambda}(\mathscr{B}(\lambda))=\left\{\tilde{b} \in \mathscr{B}(\infty) \mid \varepsilon_{i}^{*}(\tilde{b}) \leq\left\langle h_{i}, \lambda\right\rangle \text { for all } i \in I\right\} .
$$

Proposition 3.22 ([Kas93b, Lemma 5.1.1]). For $i \in I, \lambda \in P, b \in \mathscr{B}(\lambda)$ and $\tilde{b} \in \mathscr{B}(\infty)$, we have

$$
\begin{aligned}
e_{i}^{\left(\varepsilon_{i}(b)\right)} \cdot G_{\lambda}^{\mathrm{up}}(b) & =G_{\lambda}^{\mathrm{up}}\left(\tilde{e}_{i}^{\varepsilon_{i}(b)} b\right) & e_{i}^{(k)} \cdot G_{\lambda}^{\mathrm{up}}(b) & =0 \text { if } k>\varepsilon_{i}(b), \\
f_{i}^{\left(\varphi_{i}(b)\right)} \cdot G_{\lambda}^{\mathrm{up}}(b) & =G_{\lambda}^{\mathrm{up}}\left(\tilde{f}_{i}^{\varphi_{i}(b)} b\right) & f_{i}^{(k)} \cdot G_{\lambda}^{\mathrm{up}}(b) & =0 \text { if } k>\varphi_{i}(b), \\
\left(e_{i}^{\prime}\right)^{\left(\varepsilon_{i}(\tilde{b})\right)} G^{\mathrm{up}}(\tilde{b}) & =\left(1-q_{i}^{2}\right)^{\varepsilon_{i}(\tilde{b})} G^{\mathrm{up}}\left(\tilde{e}_{i}^{\varepsilon_{i}(\tilde{b})} \tilde{b}\right) & \left(e_{i}^{\prime}\right)^{(k)} G^{\mathrm{up}}(\tilde{b}) & =0 \text { if } k>\varepsilon_{i}(\tilde{b}), \\
\left.{ }_{i} e^{\prime}\right)^{\left(\varepsilon_{i}^{*}(\tilde{b})\right)} G^{\mathrm{up}}(\tilde{b}) & =\left(1-q_{i}^{2}\right)^{\varepsilon_{i}^{*}(\tilde{b})} G^{\mathrm{up}}\left(\left(\tilde{e}_{i}^{*}\right)^{\varepsilon_{i}^{*}(\tilde{b})} \tilde{b}\right) & \left({ }_{i} e^{\prime}\right)^{(k)} G^{\mathrm{up}}(\tilde{b}) & =0 \text { if } k>\varepsilon_{i}^{*}(\tilde{b}) .
\end{aligned}
$$

Here $\left(e_{i}^{\prime}\right)^{(n)}:=\left(e_{i}^{\prime}\right)^{n} /[n]_{i}$ ! and $\left({ }_{i} e^{\prime}\right)^{(n)}:=\left({ }_{i} e^{\prime}\right)^{n} /[n]_{i}$ ! for $n \in \mathbb{Z}_{\geq 0}$.

3.4. Quantum unipotent subgroups. In this subsection, we review the quantum unipotent subgroup $\mathbf{A}_{q}\left[N_{-}(w)\right]$ which is a quantum analogue of the coordinate ring $\mathbb{C}\left[N_{-}(w)\right]$ of the unipotent subgroup $N_{-}(w)$ associated with $w \in W$. See Theorem 3.29 below for the precise statement.

Definition 3.23. Following Lusztig Lus10, Section 37.1.3], we define the $\mathbb{Q}(q)$-algebra automorphism $T_{i}: \mathbf{U}_{q} \rightarrow \mathbf{U}_{q}$ for $i \in I$ by the following formulae:

$$
\begin{aligned}
T_{i}\left(q^{h}\right) & =q^{s_{i}(h)}, \\
T_{i}\left(e_{j}\right) & = \begin{cases}-f_{i} t_{i} & \text { for } j=i, \\
\sum_{r+s=-\left\langle h_{i}, \alpha_{j}\right\rangle}(-1)^{r} q_{i}^{-r} e_{i}^{(s)} e_{j} e_{i}^{(r)} & \text { for } j \neq i,\end{cases} \\
T_{i}\left(f_{j}\right) & = \begin{cases}-t_{i}^{-1} e_{i} & \text { for } j=i, \\
\sum_{r+s=-\left\langle h_{i}, \alpha_{j}\right\rangle}(-1)^{r} q_{i}^{r} f_{i}^{(r)} f_{j} f_{i}^{(s)} & \text { for } j \neq i .\end{cases}
\end{aligned}
$$

Its inverse map is given by

$$
\begin{aligned}
T_{i}^{-1}\left(q^{h}\right) & =q^{s_{i}(h)}, \\
T_{i}^{-1}\left(e_{j}\right) & = \begin{cases}-t_{i}^{-1} f_{i}(-1)^{r} q_{i}^{-r} e_{i}^{(r)} e_{j} e_{i}^{(s)} & \text { for } j \neq i, \\
\sum_{r+s=-\left\langle h_{i}, \alpha_{j}\right\rangle} j,\end{cases} \\
T_{i}^{-1}\left(f_{j}\right) & = \begin{cases}-e_{i} t_{i} & \text { for } j=i, \\
\sum_{r+s=-\left\langle h_{i}, \alpha_{j}\right\rangle}(-1)^{r} q_{i}^{r} f_{i}^{(s)} f_{j} f_{i}^{(r)} & \text { for } j \neq i .\end{cases}
\end{aligned}
$$

The maps $T_{i}$ and $T_{i}^{-1}$ are denoted by $T_{i, 1}^{\prime \prime}$ and $T_{i,-1}^{\prime}$ respectively in [Lus10]. 
It is known that $\left\{T_{i}\right\}_{i \in I}$ satisfies the braid relations, that is, for $w \in W$, the $\mathbb{Q}(q)$-algebra automorphism $T_{w}:=T_{i_{1}} \cdots T_{i_{\ell}}: \mathbf{U}_{q} \rightarrow \mathbf{U}_{q}$ does not depend on the choice of $\left(i_{1}, \ldots, i_{\ell}\right) \in I(w)$ (recall (2.1)). See [Lus10, Chapter 39].

Definition 3.24. (1) For $w \in W$, we set $\mathbf{U}_{q}^{-}(w):=\mathbf{U}_{q}^{-} \cap T_{w}\left(\mathbf{U}_{\bar{q}}^{\geq 0}\right)$. These subalgebras of $\mathbf{U}_{q}^{-}$are called quantum nilpotent subalgebras.

(2) Let $w \in W$ and $\boldsymbol{i}=\left(i_{1}, \cdots, i_{\ell}\right) \in I(w)$. For $\boldsymbol{c}=\left(c_{1}, \cdots, c_{\ell}\right) \in \mathbb{Z}_{\geq 0}^{\ell}$, we set

$$
\begin{aligned}
F^{\text {low }}(\boldsymbol{c}, \boldsymbol{i}) & :=f_{i_{1}}^{\left(c_{1}\right)} T_{i_{1}}\left(f_{i_{2}}^{\left(c_{2}\right)}\right) \cdots\left(T_{i_{1}} \cdots T_{i_{\ell-1}}\right)\left(f_{i_{\ell}}^{\left(c_{\ell}\right)}\right), \\
F^{\mathrm{up}}(\boldsymbol{c}, \boldsymbol{i}) & :=F^{\mathrm{low}}(\boldsymbol{c}, \boldsymbol{i}) /\left(F^{\mathrm{low}}(\boldsymbol{c}, \boldsymbol{i}), F^{\mathrm{low}}(\boldsymbol{c}, \boldsymbol{i})\right)_{L} .
\end{aligned}
$$

Proposition 3.25 ([DKP95, Proposition 2.2], [BCP99, Proposition 2.3], Lus10, Proposition 38.2.3]). (1) $F^{\text {low }}(\boldsymbol{c}, \boldsymbol{i}) \in \mathbf{U}_{q}^{-}(w)$ for $\boldsymbol{c} \in \mathbb{Z}_{\geq 0}^{\ell}$.

(2) $\left\{F^{\text {low }}(\boldsymbol{c}, \boldsymbol{i})\right\}_{\boldsymbol{c} \in \mathbb{Z}_{\geq 0}^{\ell}}$ is an orthogonal basis of $\mathbf{U}_{q}^{-}(w)$ with respect to the pairing $(,)_{L}$, more precisely, we have

$$
\left(F^{\mathrm{low}}(\boldsymbol{c}, \boldsymbol{i}), F^{\mathrm{low}}\left(\boldsymbol{c}^{\prime}, \boldsymbol{i}\right)\right)_{L}=\delta_{\boldsymbol{c}, \boldsymbol{c}^{\prime}} \prod_{k=1}^{\ell} \prod_{j=1}^{c_{k}}\left(1-q_{i_{k}}^{2 j}\right)^{-1},
$$

here $\boldsymbol{i}=\left(i_{1, \ldots,}, i_{\ell}\right)$.

By Proposition (3.25), $\left\{F^{\text {up }}(\boldsymbol{c}, \boldsymbol{i})\right\}_{\boldsymbol{c} \in \mathbb{Z}_{\geq 0}^{\ell}}$ is also an orthogonal basis of $\mathbf{U}_{q}^{-}(w)$ with respect to the Lusztig pairing. The basis $\left\{F^{\text {low }}(\boldsymbol{c}, \boldsymbol{i})\right\}_{\boldsymbol{c} \in \mathbb{Z}_{\geq 0}^{\ell}}$ is called the (lower) Poincaré-BirkhoffWitt type basis associated with $\boldsymbol{i} \in I(w)$, and the basis $\left\{F^{\text {up }}(\boldsymbol{c}, \boldsymbol{i})\right\}_{\boldsymbol{c}}$ is called the dual (or upper) Poincaré-Birkhoff-Witt type basis.

Definition 3.26. For $w \in W$, we set

$$
\begin{aligned}
\mathbf{U}_{q}^{+}(w) & :=\left(\mathbf{U}_{q}^{-}(w)\right)^{\vee}, \\
\mathbf{A}_{q}\left[N_{-}(w)\right] & :=*\left(\mathbf{U}_{q}^{-}(w)\right) .
\end{aligned}
$$

We call $\mathbf{A}_{q}\left[N_{-}(w)\right]$ a quantum unipotent subgroup. The quantum unipotent subgroup has a $Q_{-}$-graded algebra structure induced from that of $\mathbf{U}_{q}^{-}$. Note that $\varphi\left(\mathbf{A}_{q}\left[N_{-}(w)\right]\right)=\mathbf{U}_{q}^{+}(w)$.

Proposition 3.27 ([Kim12, Theorem 4.25, Theorem 4.29]). Let $w \in W$ and $\boldsymbol{i} \in I(w)$. Then the following hold:

(1) $\mathbf{U}_{q}^{-}(w) \cap \mathbf{B}^{\mathrm{up}}$ is a basis of $\mathbf{U}_{q}^{-}(w)$.

(2) each element $G^{\mathrm{up}}(b)$ of $\mathbf{U}_{q}^{-}(w) \cap \mathbf{B}^{\mathrm{up}}$ is characterized by the following conditions:

(DCB1) $\sigma\left(G^{\mathrm{up}}(b)\right)=G^{\mathrm{up}}(b)$, and

(DCB2) $G^{\text {up }}(b)=F^{\text {up }}(\boldsymbol{c}, \boldsymbol{i})+\sum_{\boldsymbol{c}^{\prime}<\boldsymbol{c}} d_{\boldsymbol{c}, \boldsymbol{c}^{\prime}}^{\boldsymbol{i}} F^{\text {up }}\left(\boldsymbol{c}^{\prime}, \boldsymbol{i}\right)$ with $d_{\boldsymbol{c}, \boldsymbol{c}^{\prime}}^{\boldsymbol{i}} \in q \mathbb{Z}[q]$ for some $\boldsymbol{c} \in \mathbb{Z}_{\geq 0}^{\ell}$.

Here $<$ denotes the left lexicographic order on $\mathbb{Z}_{\geq 0}^{\ell}$, that is, we write $\left(c_{1}, \ldots, c_{\ell}\right)<\left(c_{1}^{\prime}, \ldots, c_{\ell}^{\prime}\right)$ if and only if there exists $k \in\{1, \ldots, \ell\}$ such that $c_{1}=c_{1}^{\prime}, \ldots, c_{k-1}=c_{k-1}^{\prime}$ and $c_{k}<c_{k}^{\prime}$.

Definition 3.28. Proposition 3.27 (2) says that each $F^{\text {up }}(\boldsymbol{c}, \boldsymbol{i})$ determines a unique dual canonical basis element $G^{\text {up }}(b)$ in $\mathbf{U}_{q}^{-}(w)$. We write the corresponding element of $\mathscr{B}(\infty)$ as $b(\boldsymbol{c}, \boldsymbol{i})$. Then

$$
\mathbf{U}_{q}^{-}(w) \cap \mathbf{B}^{\text {up }}=\left\{G^{\text {up }}(b(\boldsymbol{c}, \boldsymbol{i}))\right\}_{\boldsymbol{c} \in \mathbb{Z}_{\geq 0}^{\ell}} .
$$


Write $\mathscr{B}\left(\mathbf{U}_{q}^{-}(w)\right):=\{b(\boldsymbol{c}, \boldsymbol{i})\}_{\boldsymbol{c} \in \mathbb{Z}_{>0}^{\ell}}$. Note that $\mathscr{B}\left(\mathbf{U}_{q}^{-}(w)\right)$ does not depend on the choice of $\boldsymbol{i} \in I(w)$. Set $b_{-1}(\boldsymbol{c}, \boldsymbol{i}):=*(b(\boldsymbol{c}, \overline{\boldsymbol{i}}))$. Then $\mathbf{A}_{q}\left[N_{-}(w)\right] \cap \mathbf{B}^{\mathrm{up}}=\left\{G^{\mathrm{up}}\left(b_{-1}(\boldsymbol{c}, \boldsymbol{i})\right)\right\}_{\boldsymbol{c} \in \mathbb{Z}_{\geq 0}^{\ell}}$.

The following is the specialization result for the quantum unipotent subgroup which justifies the notation $\mathbf{A}_{q}\left[N_{-}(w)\right]$.

Theorem 3.29 ([Kim12, Theorem 4.44]). For $w \in W$, we set $\mathbf{A}_{\mathbb{Q}\left[q^{ \pm 1}\right]}\left[N_{-}(w)\right]:=\mathbf{A}_{\mathbb{Q}\left[q^{ \pm 1}\right]}\left[\boldsymbol{N}_{-}\right] \cap$ $\mathbf{A}_{q}\left[N_{-}(w)\right]$. Then we have

$$
\mathbf{A}_{\mathbb{Q}\left[q^{ \pm 1}\right]}\left[N_{-}(w)\right] \otimes_{\mathcal{A}} \mathbb{C} \simeq \mathbb{C}\left[N_{-}(w)\right],
$$

here we regard $\mathbb{C}$ as an $\mathcal{A}$-module via $q^{ \pm 1} \mapsto 1$.

Remark 3.30. In [Kim12], the $\mathcal{A}$-form $\mathbf{A}_{\mathbb{Q}\left[q^{ \pm 1}\right]}\left[N_{-}(w)\right]$ is defined by the non-degenerate bilinear form $(,)_{K}$ on $\mathbf{U}_{q}^{-}(\mathfrak{g})$ with $\left(f_{i}, f_{i}\right)_{K}=1$ for $i \in I$. But this specialization result is not affected since the structure constants with respect to the dual canonical bases defined by $(,)_{L}$ and $(,)_{K}$ are the same. For more details, see [Kim12, Lemma 2.12].

3.5. Quantum closed unipotent cells. In this section, we review the definition of quantum closed unipotent cells. For more details, see [Kim12, Section 5].

Definition 3.31. Let $M=\bigoplus_{\mu \in P} M_{\mu}$ be an integrable $\mathbf{U}_{q}$-module (i.e., $e_{i}$ and $f_{i}$ act locally nilpotently on $M$ for all $i \in I$ ) with weight space decomposition. For $i \in I$, there exists a $\mathbb{Q}(q)$-linear automorphism $T_{i}$ of $M$ given by

$$
\begin{aligned}
& T_{i}(m):=\sum_{-a+b-c=\left\langle h_{i}, \mu\right\rangle}(-1)^{b} q_{i}^{-a c+b} e_{i}^{(a)} f_{i}^{(b)} e_{i}^{(c)} \cdot m \\
& T_{i}^{-1}(m)=\sum_{a-b+c=\left\langle h_{i}, \mu\right\rangle}(-1)^{b} q_{i}^{a c-b} f_{i}^{(a)} e_{i}^{(b)} f_{i}^{(c)} \cdot m
\end{aligned}
$$

for $m \in M_{\mu}, \mu \in P$. The maps $T_{i}$ and $T_{i}^{-1}$ are denoted by $T_{i}=T_{i, 1}^{\prime \prime}$ and $T_{i}=T_{i,-1}^{\prime}$ respectively in [Lus10, Chapter 5].

The following propositions are fundamental properties of $T_{i}$. See, for example, Lus10, Chapter 37, 39]:

Proposition 3.32. Let $M$ be an integrable $\mathbf{U}_{q}$-module. (See Definition 3.31.) (1) For $x \in \mathbf{U}_{q}$ and $m \in M$, we have $T_{i}(x \cdot m)=T_{i}(x) \cdot T_{i}(m)$.

(2) For $w \in W$, the composite map $T_{w}:=T_{i_{1}} \cdots T_{i_{\ell}}: M \rightarrow M$ does not depend on the choice of $\left(i_{1}, \ldots, i_{\ell}\right) \in I(w)$.

(3) For $\mu \in P$ and $w \in W, T_{w}$ maps $M_{\mu}$ to $M_{w \mu}$.

Proposition 3.33. Let $\lambda \in P_{+}, w \in W$ and $\boldsymbol{i}=\left(i_{1}, \ldots, i_{\ell}\right) \in I(w)$. Recall that $u_{\lambda}$ is a highest weight vector of $V(\lambda)$ (Definition 3.13). Then we have

$$
u_{w \lambda}:=\left(T_{w^{-1}}\right)^{-1}\left(u_{\lambda}\right)=f_{i_{1}}^{\left(a_{1}\right)} f_{i_{2}}^{\left(a_{2}\right)} \ldots f_{i_{\ell}}^{\left(a_{\ell}\right)} \cdot u_{\lambda},
$$

where $a_{1}=\left\langle h_{i_{1}}, s_{i_{2}} \ldots s_{i_{\ell}} \lambda\right\rangle, \ldots, a_{\ell}=\left\langle h_{i_{\ell}}, \lambda\right\rangle$. Note that $a_{1}, \ldots, a_{\ell} \in \mathbb{Z}_{\geq 0}$.

It is easy to show that $\left(u_{w \lambda}, u_{w \lambda}\right)_{\lambda}^{\varphi}=1$ for $\lambda \in P_{+}$and $w \in W$. Actually, the vector $u_{w \lambda}$ belongs to $\mathbf{B}^{\text {low }}(\lambda)$ and $\mathbf{B}^{\mathrm{up}}(\lambda)$ [Kas93a, subsection 3.2]. 
Proposition 3.34 ([Kas93a, Proposition 3.2.3, 3.2.5]). (1) For $\lambda \in P_{+}, w \in W$ and $\boldsymbol{i}=$ $\left(i_{1}, \cdots, i_{\ell}\right) \in I(w)$, we set

$$
\mathscr{B}_{w}(\lambda):=\left\{\widetilde{f}_{i_{1}}^{a_{1}} \cdots \widetilde{f}_{i_{\ell}}^{a_{\ell}} u_{\lambda} \mid \boldsymbol{a}=\left(a_{1}, \cdots, a_{\ell}\right) \in \mathbb{Z}_{\geq 0}^{\ell}\right\} \backslash\{0\} \subset \mathscr{B}(\lambda)
$$

and $V_{w}(\lambda):=\mathbf{U}_{q}^{+} \cdot u_{w \lambda}$. Then we have

$$
V_{w}(\lambda)=\bigoplus_{b \in \mathscr{B}_{w}(\lambda)} \mathbb{Q}(q) G_{\lambda}^{\text {low }}(b) .
$$

In particular, $\mathscr{B}_{w}(\lambda)$ is independent of the choice of $\boldsymbol{i} \in I(w)$.

(2) For $w \in W$ and $\boldsymbol{i}=\left(i_{1}, \cdots, i_{\ell}\right) \in I(w)$, we set

$$
\mathscr{B}_{w}(\infty):=\left\{\tilde{f}_{i_{1}}^{a_{1}} \cdots \widetilde{f}_{i_{\ell}}^{a_{\ell}} u_{\infty} \mid \boldsymbol{a}=\left(a_{1}, \cdots, a_{\ell}\right) \in \mathbb{Z}_{\geq 0}^{\ell}\right\}
$$

and $\mathbf{U}_{w, q}^{-}:=\sum_{a_{1}, \cdots, a_{\ell} \in \mathbb{Z}_{\geq 0}} \mathbb{Q}(q) f_{i_{1}}^{a_{1}} \cdots f_{i_{\ell}}^{a_{\ell}}$. Then we have

$$
\mathbf{U}_{w, q}^{-}=\bigoplus_{b \in \mathscr{B}_{w}(\infty)} \mathbb{Q}(q) G^{\text {low }}(b) .
$$

Moreover $\mathscr{B}_{w}(\infty)$ and $\mathbf{U}_{w, q}^{-}$are independent of the choice of $\boldsymbol{i} \in I(w)$.

Remark 3.35. By Proposition $3.34(2), \mathbf{U}_{w, q}^{-} \cap \mathbf{U}_{\mathcal{A}}^{-}=\bigoplus_{b \in \mathscr{B}_{w}(\infty)} \mathcal{A} G^{\text {low }}(b)=: \mathbf{U}_{w, \mathcal{A}}^{-}$. Hence,

$$
\mathbf{U}_{w, \mathcal{A}}^{-} \otimes_{\mathcal{A}} \mathbb{C} \stackrel{\sim}{\rightarrow} \mathbf{U}_{w}^{-} .
$$

Set $\mathbf{U}_{w}^{-}:=\sum_{a_{1}, \cdots, a_{\ell}} \mathbb{C} f_{i_{1}}^{a_{1}} \cdots f_{i_{\ell}}^{a_{\ell}} \subset \mathbf{U}\left(\mathfrak{n}_{-}\right)$for a reduced expression $\boldsymbol{i} \in I(w)$.

For more details on Demazure modules and its crystal basis, see Kashiwara [Kas93a].

Remark 3.36. We have

$$
\bigcup_{\lambda \in P_{+}} \bar{\jmath}_{\lambda}\left(\mathscr{B}_{w}(\lambda)\right)=\mathscr{B}_{w}(\infty)
$$

See also Theorem 3.48 ,

Definition 3.37 ([Kim12, 5.1.3]). Let $w \in W$. Set

$$
\left(\mathbf{U}_{w, q}^{-}\right)^{\perp}:=\left\{x \in \mathbf{U}_{q}^{-} \mid\left(x, \mathbf{U}_{w, q}^{-}\right)_{L}=0\right\} .
$$

Then, by $\Delta\left(\mathbf{U}_{w, q}^{-}\right) \subset \mathbf{U}_{w, q}^{-} \mathbf{U}_{q}^{0} \otimes \mathbf{U}_{w, q}^{-}$and Lemma 3.8, $\left(\mathbf{U}_{w, q}^{-}\right)^{\perp}$ is a two-sided ideal of $\mathbf{U}_{q}^{-}$. Hence we obtain a $\mathbb{Q}(q)$-algebra

$$
\mathbf{A}_{q}\left[N_{-} \cap X_{w}\right]:=\mathbf{U}_{q}^{-} /\left(\mathbf{U}_{w, q}^{-}\right)^{\perp},
$$

called the quantum closed unipotent cell. The quantum closed unipotent cell has a $Q_{- \text {-graded }}$ algebra structure induced from that of $\mathbf{U}_{q}^{-}$. Note that

$$
\left(\mathbf{U}_{w, q}^{-}\right)^{\perp}=\bigoplus_{b \in \mathscr{B}(\infty) \backslash \mathscr{B}_{w}(\infty)} \mathbb{Q}(q) G^{\mathrm{up}}(b) .
$$

Describe the canonical projection $\mathbf{U}_{q}^{-} \rightarrow \mathbf{A}_{q}\left[N_{-} \cap X_{w}\right]$ as $x \mapsto[x]$. The element $[x]$ clearly depends on $w$, however, we omit to write $w$ because it will cause no confusion below.

Remark 3.38. In [Kim12, 5.1.3], $\mathbf{A}_{q}\left[N_{-} \cap X_{w}\right]$ is denoted by $\mathcal{O}_{q}\left[\overline{N_{w}}\right]$. 
We set the $\mathcal{A}$-form $\mathbf{A}_{\mathbb{Q}\left[q^{ \pm 1}\right]}\left[N_{-} \cap X_{w}\right]$ of $\mathbf{A}_{q}\left[N_{-} \cap X_{w}\right]$ by

$$
\mathbf{A}_{\mathbb{Q}\left[q^{ \pm 1}\right]}\left[N_{-} \cap X_{w}\right]:=\mathbf{A}_{\mathbb{Q}\left[q^{ \pm 1}\right]}\left[\boldsymbol{N}_{-}\right] /\left(\left(\mathbf{U}_{w, q}^{-}\right)^{\perp} \cap \mathbf{A}_{\mathbb{Q}\left[q^{ \pm 1}\right]}\left[\boldsymbol{N}_{-}\right]\right) .
$$

Note that we have

$$
\left(\mathbf{U}_{w, q}^{-}\right)^{\perp} \cap \mathbf{A}_{\mathbb{Q}\left[q^{ \pm 1}\right]}\left[\boldsymbol{N}_{-}\right]=\bigoplus_{b \in \mathscr{B}(\infty) \backslash \mathscr{B}_{w}(\infty)} \mathcal{A} G^{\text {up }}(b) .
$$

The following is the specialization result for quantum closed unipotent cell which justifies the notation $\mathbf{A}_{q}\left[N_{-} \cap X_{w}\right]$.

Theorem 3.39. For $w \in W$, we have

$$
\mathbf{A}_{\mathbb{Q}\left[q^{ \pm 1}\right]}\left[N_{-} \cap X_{w}\right] \otimes_{\mathcal{A}} \mathbb{C} \simeq \mathbb{C}\left[N_{-} \cap X_{w}\right] .
$$

here we regard $\mathbb{C}$ as an $\mathcal{A}$-module via $q^{ \pm 1} \mapsto 1$.

Proof. We have an exact sequence of $\mathcal{A}$-modules

$$
0 \rightarrow\left(\mathbf{U}_{w}^{-}\right)^{\perp} \cap \mathbf{A}_{\mathbb{Q}\left[q^{ \pm 1}\right]}\left[\boldsymbol{N}_{-}\right] \rightarrow \mathbf{A}_{\mathbb{Q}\left[q^{ \pm 1}\right]}\left[\boldsymbol{N}_{-}\right] \rightarrow \mathbf{A}_{\mathbb{Q}\left[q^{ \pm 1}\right]}\left[N_{-} \cap X_{w}\right] \rightarrow 0
$$

here the second map is the inclusion and the third map is the projection. Moreover, $\left(\mathbf{U}_{w}^{-}\right)^{\perp} \cap$ $\mathbf{A}_{\mathbb{Q}\left[q^{ \pm 1}\right]}\left[\boldsymbol{N}_{-}\right]$and $\mathbf{A}_{\mathbb{Q}\left[q^{ \pm 1}\right]}\left[\boldsymbol{N}_{-}\right]$are free $\mathcal{A}$-modules and an $\mathcal{A}$-basis of the former can be chosen as the subset of that of the latter (see $(3.6)$ ). Therefore $\mathbf{A}_{\mathbb{Q}\left[q^{ \pm 1}\right]}\left[N_{-} \cap X_{w}\right]$ is also a free $\mathcal{A}$ module (more precisely, $\mathbf{A}_{\mathbb{Q}\left[q^{ \pm 1}\right]}\left[N_{-} \cap X_{w}\right]$ admits the projected dual canonical basis), and we have

$$
\begin{aligned}
\mathbf{A}_{\mathbb{Q}\left[q^{ \pm 1}\right]}\left[N_{-} \cap X_{w}\right] \otimes_{\mathcal{A}} \mathbb{C} & \simeq \frac{\mathbf{A}_{\mathbb{Q}\left[q^{ \pm 1}\right]}\left[\boldsymbol{N}_{-}\right] \otimes_{\mathcal{A}} \mathbb{C}}{\left(\left(\mathbf{U}_{w}^{-}\right)^{\perp} \cap \mathbf{A}_{\mathbb{Q}\left[q^{ \pm 1}\right]}\left[\boldsymbol{N}_{-}\right]\right) \otimes_{\mathcal{A}} \mathbb{C}} \\
& =\mathbf{U}\left(\mathfrak{n}_{-}\right)_{\mathrm{gr}}^{*} /\left(\mathbf{U}_{w}^{-}\right)^{\perp} \simeq \mathbb{C}\left[N_{-} \cap X_{w}\right]
\end{aligned}
$$

here the last isomorphism follows from Proposition 2.16.

\subsection{Unipotent quantum matrix coefficients.}

Definition 3.40. For $\lambda \in P_{+}$and $u, u^{\prime} \in V(\lambda)$, define the element $D_{u, u^{\prime}} \in \mathbf{U}_{q}^{-}$by

$$
\left(D_{u, u^{\prime}}, x\right)_{L}=\left(u, x \cdot u^{\prime}\right)_{\lambda}^{\varphi}
$$

for all $x \in \mathbf{U}_{q}^{-}$. We call an element of this form a unipotent quantum matrix coefficient. Note that $\operatorname{wt}\left(D_{u, u^{\prime}}\right)=\operatorname{wt} u-\operatorname{wt} u^{\prime}$ for weight vectors $u, u^{\prime} \in V(\lambda)$. For $w, w^{\prime} \in W$, we write

$$
D_{w \lambda, w^{\prime} \lambda}:=D_{u_{w \lambda}, u_{w^{\prime} \lambda}}
$$

which is called a unipotent quantum minor. See [Kim12, Section 6].

Definition 3.41. Let $\lambda \in P_{+}$. Define a surjective $\mathbb{Q}(q)$-linear map $\pi_{w \lambda}^{\vee}: \mathbf{U}_{q}^{-} \rightarrow V_{w}(\lambda)$ by

$$
\pi_{w \lambda}^{\vee}(y)=(y)^{\vee} \cdot u_{w \lambda}
$$

Proposition 3.42 ([Lus10, Proposition 25.2.6], [Kas94, 8.2.2 (iii), (iv)]). Let $\lambda \in P_{+}$and $w \in W$. Then there exists a surjective map $\pi_{w \lambda}^{\vee}: \mathscr{B}(\infty) \rightarrow \mathscr{B}_{w}(\lambda) \coprod\{0\}$ such that

$$
\pi_{w \lambda}^{\vee}\left(G^{\text {low }}(b)\right)=G_{\lambda}^{\text {low }}\left(\pi_{w \lambda}^{\vee}(b)\right)
$$

for $b \in \mathscr{B}(\infty)$, here $G_{\lambda}^{\text {low }}(0)=0$. Moreover, $\pi_{w \lambda}^{\vee}$ induces a bijection $\left(\pi_{w \lambda}^{\vee}\right)^{-1}\left(\mathscr{B}_{w}(\lambda)\right) \rightarrow$ $\mathscr{B}_{w}(\lambda)$. 
Definition 3.43. Let $\lambda \in P_{+}$and $w \in W$. Set $V_{w}(\lambda)^{\perp}:=\left\{u \in V(\lambda) \mid\left(u, V_{w}(\lambda)\right)_{\lambda}^{\varphi}=0\right\}$. Define $j_{w \lambda}^{\vee}: V(\lambda) / V_{w}(\lambda)^{\perp} \hookrightarrow \mathbf{U}_{q}^{-}$as the dual homomorphism of $\pi_{w \lambda}^{\vee}$ given by the nondegenerate bilinear forms $(,)_{\lambda}^{\varphi}: V(\lambda) \times V(\lambda) \rightarrow \mathbb{Q}(q)$ and $(,)_{L}: \mathbf{U}_{q}^{-} \times \mathbf{U}_{q}^{-} \rightarrow \mathbb{Q}(q)$, that is,

$$
\begin{gathered}
\left(j_{w \lambda}^{\vee}(u), y\right)_{L}=\left(u, \pi_{w \lambda}^{\vee}(y)\right)_{\lambda}^{\varphi}\left(=\left(u, y^{\vee} \cdot u_{w \lambda}\right)_{\lambda}^{\varphi}=\left(\varphi\left(y^{\vee}\right) \cdot u, u_{w \lambda}\right)_{\lambda}^{\varphi}\right) . \\
V(\lambda) /\left(V_{w}(\lambda)\right)^{\perp} \stackrel{j_{w \lambda}^{\vee}}{\longrightarrow} \mathbf{U}_{q}^{-} \\
\left(V_{w}(\lambda)\right)_{\mathrm{gr}}^{*} \stackrel{\left(\pi_{w \lambda}^{\vee}\right)_{\mathrm{gr}}^{*}}{\longrightarrow}\left(\mathbf{U}_{q}^{-}\right)_{\mathrm{gr}}^{*}
\end{gathered}
$$

In the following, the map $V(\lambda) \rightarrow \mathbf{U}_{q}^{-}$given by $u \mapsto j_{w \lambda}^{\vee}\left(p_{w}(u)\right)$ is also denoted by $j_{w \lambda}^{\vee}$, here $p_{w}$ denotes the canonical projection $V(\lambda) \rightarrow V(\lambda) / V_{w}(\lambda)^{\perp}$.

The following proposition immediately follows from Proposition 3.42 ,

Proposition 3.44. Let $\lambda \in P_{+}$and $w \in W$. Then there is an injective map $\bar{\jmath}_{w \lambda}^{\vee}: \mathscr{B}_{w}(\lambda) \hookrightarrow$ $\mathscr{B}(\infty)$ such that

$$
\left(G_{\lambda}^{\mathrm{up}}(b), G^{\mathrm{low}}\left(b^{\prime}\right)^{\vee} \cdot u_{w \lambda}\right)_{\lambda}^{\varphi}=\delta_{b^{\prime}, \bar{j}_{w \lambda}^{\vee}(b)}
$$

for any $b \in \mathscr{B}_{w}(\lambda)$ and $b^{\prime} \in \mathscr{B}(\infty)$. That is, we have $j_{w \lambda}^{\vee}\left(G_{\lambda}^{\mathrm{up}}(b)\right)=G^{\mathrm{up}}\left(\bar{\jmath}_{w \lambda}^{\vee}(b)\right)$.

Remark 3.45. Let $\lambda \in P_{+}$and $w \in W$. Then,

- wt $\bar{\jmath}_{w \lambda}^{\vee}(b)=-\mathrm{wt} b+w \lambda$ for $b \in \mathscr{B}_{w}(\lambda)$, and

- $\bar{\jmath}_{w \lambda}^{\vee}\left(\pi_{w \lambda}^{\vee}(b)\right)=b$ for $b \in\left(\pi_{w \lambda}^{\vee}\right)^{-1}\left(\mathscr{B}_{w}(\lambda)\right)$.

Proposition 3.46. Let $\lambda \in P_{+}$and $w \in W$. Then the following hold:

(1) $D_{G_{\lambda}^{\mathrm{up}}(b), u_{\lambda}}=G^{\mathrm{up}}\left(\bar{\jmath}_{\lambda}(b)\right)$ for all $b \in \mathscr{B}(\lambda)$,

(2) $D_{u_{w \lambda}, G_{\lambda}^{\mathrm{up}}(b)}=G^{\mathrm{up}}\left(* \bar{\jmath}_{w \lambda}^{\vee}(b)\right)$ for all $b \in \mathscr{B}_{w}(\lambda)$, and

(3) $D_{u_{w \lambda}, G_{\lambda}^{\text {up }}(b)}=0$ for all $b \in \mathscr{B}(\lambda) \backslash \mathscr{B}_{w}(\lambda)$.

Proof. The equality (1) follows immediately by Proposition 3.16 . For $y \in \mathbf{U}_{q}^{-}$, we have

$$
\begin{aligned}
\left(D_{u_{w \lambda}, G_{\lambda}^{\mathrm{up}}(b)}, y\right)_{L} & =\left(u_{w \lambda}, y \cdot G_{\lambda}^{\mathrm{up}}(b)\right)_{\lambda}^{\varphi} \\
& =\left(G_{\lambda}^{\mathrm{up}}(b),(*(y))^{\vee} \cdot u_{w \lambda}\right)_{\lambda}^{\varphi} \\
& =\left(G^{\mathrm{up}}\left(\bar{\jmath}_{w \lambda}^{\vee}(b)\right), *(y)\right)_{L} \\
& =\left(G^{\mathrm{up}}\left(* \bar{\jmath}_{w \lambda}^{\vee}(b)\right), y\right)_{L} .
\end{aligned}
$$

This completes the proof of (2). The assertion (3) follows from the similar calculation and Proposition 3.34,

Proposition 3.47 ([Kim12, Corollary 6.4]). Let $w \in W$ and $\boldsymbol{i}=\left(i_{1}, \ldots, i_{\ell}\right) \in I(w)$. For $i \in I$, define $\boldsymbol{n}^{(i)}=\left(n_{1}^{(i)}, \ldots, n_{\ell}^{(i)}\right) \in \mathbb{Z}_{\geq 0}^{\ell}$ by

$$
n_{k}^{(i)}= \begin{cases}1 & \text { if } i_{k}=i, \\ 0 & \text { otherwise. }\end{cases}
$$

For $\lambda \in P_{+}$, set $\boldsymbol{n}^{\lambda}:=\sum_{i \in I}\left\langle\lambda, h_{i}\right\rangle \boldsymbol{n}^{(i)}$. Then we have

$$
D_{w \lambda, \lambda}=G^{\mathrm{up}}\left(b_{-1}\left(\boldsymbol{n}^{\lambda}, \boldsymbol{i}\right)\right) .
$$


3.7. Kumar-Peterson identities. We investigate the map $\bar{\jmath}_{w \lambda}^{\vee}$ a little more. Kumar and Peterson studied the identity which expresses the $H$-characters of the coordinate $\operatorname{ring} \mathbb{C}\left[X_{w} \cap \boldsymbol{U}_{v}\right]$ of the intersection $X_{w} \cap \boldsymbol{U}_{v}$ of Schubert varieties $X_{w}$ and $v$-translates of the open cell $\boldsymbol{U}_{v}$ as the limit of a family of "twisted" characters of Demazure modules in general Kac-Moody Lie algebras, see Kumar Kum02, Theorem 12.1.3]. In the special case with $v=w$, it reduces to the case of Schubert cells, that is, we have $X_{w} \cap \boldsymbol{U}_{w}=\stackrel{\circ}{X}_{w}$ (see Kumar Kum02, Lemma 7.3.10]) and the following equality can be considered as a crystalized Kumar-Peterson identity.

Theorem 3.48. We have

$$
\bigcup_{\lambda \in P_{+}} \bar{\jmath}_{w \lambda}^{\vee}\left(\mathscr{B}_{w}(\lambda)\right)=\mathscr{B}\left(\mathbf{U}_{q}^{-}(w)\right)
$$

The rest of this subsection is devoted to the proof of Theorem 3.48 .

Lemma 3.49 ([KO18, Lemma 3.19]). For $w \in W$, let $\mathbf{U}_{q}^{-}(w)^{\perp}$ be the orthogonal complement of $\mathbf{U}_{q}^{-}(w)$ with respect to $(,)_{L}$. We have an isomorphism as $\mathbb{Q}(q)$-vector spaces:

$$
\mathbf{U}_{q}^{-}(w) \otimes\left(\mathbf{U}_{q}^{-} \cap T_{w} \mathbf{U}_{q}^{-} \cap \operatorname{Ker}(\varepsilon)\right) \stackrel{\sim}{\rightarrow}\left(\mathbf{U}_{q}^{-}(w)\right)^{\perp} \subset \mathbf{U}_{q}^{-}
$$

under the multiplication $\mathbf{U}_{q}^{-}(w) \otimes\left(\mathbf{U}_{q}^{-} \cap T_{w} \mathbf{U}_{q}^{-}\right) \stackrel{\sim}{\rightarrow} \mathbf{U}_{q}^{-}$, here recall that $\varepsilon$ is the counit of $\mathbf{U}_{q}$ (see Definition 3.2).

Lemma 3.50. For $y \in \mathbf{U}_{q}^{-}(w)^{\perp}$, we have $y^{\vee} . u_{w \lambda}=0$ for all $\lambda \in P_{+}$.

Proof. By Lemma 3.49, we write $y=\sum y_{(1)} y_{(2)}$ with $y_{(1)} \in \mathbf{U}_{q}^{-}(w)$ and homogeneous elements $y_{(2)} \in \mathbf{U}_{q}^{-} \cap T_{w} \mathbf{U}_{q}^{-} \cap \operatorname{Ker}(\varepsilon)$. Then we have

$$
(y)^{\vee} \cdot u_{w \lambda}=\left(T_{w^{-1}}\right)^{-1}\left(\sum T_{w^{-1}}\left(y_{(1)}^{\vee}\right) T_{w^{-1}}\left(y_{(2)}^{\vee}\right) \cdot u_{\lambda}\right)=0
$$

because wt $\left(T_{w^{-1}}\left(y_{(2)}^{\vee}\right)\right) \in Q_{+} \backslash\{0\}$.

Proposition 3.51. We have

$$
\bigcup_{\lambda \in P_{+}} \bar{\jmath}_{w \lambda}^{\vee}\left(\mathscr{B}_{w}(\lambda)\right) \subset \mathscr{B}\left(\mathbf{U}_{q}^{-}(w)\right)
$$

Proof. Let $\pi(w): \mathbf{U}_{q}^{-} \rightarrow \mathbf{U}_{q}^{-}(w)$ be the projection with respect to the decomposition $\mathbf{U}_{q}^{-}=$ $\mathbf{U}_{q}^{-}(w) \oplus \mathbf{U}_{q}^{-}(w)^{\perp}$. Since $\mathbf{U}_{q}^{-}(w)^{\perp} \cap \mathbf{B}^{\text {low }}$ is a basis of $\mathbf{U}_{q}^{-}(w)^{\perp}$ by Proposition 3.27, we have $\pi(w)\left(G^{\text {low }}(b)\right) \neq 0$ if and only if $b \in \mathscr{B}\left(\mathbf{U}_{q}^{-}(w)\right)$ for $b \in \mathscr{B}(\infty)$. Let $b \in \bigcup_{\lambda \in P_{+}} \bar{\jmath}_{w \lambda}^{\vee}\left(\mathscr{B}_{w}(\lambda)\right)$. Then there exists $\lambda \in P_{+}$such that $\left(G^{\text {low }}(b)\right)^{\vee} . u_{w \lambda} \neq 0$. By Proposition 3.50, we have

$$
\left(G^{\text {low }}(b)\right)^{\vee} \cdot u_{w \lambda}=\left(\pi(w)\left(G^{\text {low }}(b)\right)\right)^{\vee} \cdot u_{w \lambda} .
$$

In particular, we have $\pi(w)\left(G^{\text {low }}(b)\right) \neq 0$. This completes the proof.

We prove the opposite inclusion.

Proposition 3.52. We have

$$
\mathscr{B}\left(\mathbf{U}_{q}^{-}(w)\right) \subset \bigcup_{\lambda \in P_{+}} \bar{\jmath}_{w \lambda}^{\vee}\left(\mathscr{B}_{w}(\lambda)\right) .
$$


Proof. Let $b \in \mathscr{B}\left(\mathbf{U}_{q}^{-}(w)\right)$, that is $0 \neq \pi(w)\left(G^{\text {low }}(b)\right) \in \mathbf{U}_{q}^{-}(w)$. (See the proof of Proposition 3.51,) By Proposition 3.42 and Remark 3.45, it suffices to show that $G^{\text {low }}(b)^{\vee} \cdot u_{w \lambda}=$ $\left(\pi(w)\left(G^{\text {low }}(b)\right)\right)^{\vee} . u_{w \lambda} \neq 0$ for some $\lambda \in P_{+}$. Note that $\left(\pi(w)\left(G^{\text {low }}(b)\right)\right)^{\vee} . u_{w \lambda} \neq 0$ is equivalent to $\overline{\left(\pi(w)\left(G^{\text {low }}(b)\right)\right)} \vee \cdot u_{w \lambda} \neq 0$.

By the way, we have

$$
\bar{y}^{\vee} \cdot u_{w \lambda}=\left(T_{w^{-1}}\right)^{-1}\left(\left(T_{w^{-1}} \circ \vee \circ^{-}\right)(y) \cdot u_{\lambda}\right)=\left(T_{w^{-1}}\right)^{-1}\left(\left(\vee \circ-\circ T_{w}^{-1}\right)(y) \cdot u_{\lambda}\right) .
$$

Since $y_{0}:=\pi(w)\left(G^{\text {low }}(b)\right) \in \mathbf{U}_{q}^{-} \cap T_{w} \mathbf{U}_{\bar{q}}^{\geq 0}$, we have $\left(\vee \circ^{-} \circ T_{w}^{-1}\right)\left(y_{0}\right) \in \mathbf{U}_{\bar{q}}^{\leq 0}$. It is well-known that, for $\xi \in Q_{-}$, there exists an element $\lambda \in P_{+}$such that the projection $\left(\mathbf{U}_{q}^{-}\right)_{\xi} \rightarrow V(\lambda)_{\xi+\lambda}$ given by $y \mapsto y \cdot u_{\lambda}$ is an isomorphism of vector space. Hence it can be shown that there exists $\lambda \in P_{+}$such that $\left(\vee \circ-\circ T_{w}^{-1}\right)\left(y_{0}\right) . u_{\lambda} \neq 0$.

\section{Quantum unipotent cells and the De Concini-Procesi isomorphisms}

In this section, we introduce quantum unipotent cells $\mathbf{A}_{q}\left[N_{-}^{w}\right]$ following De Concini-Procesi [DP97, and show that they are isomorphic to the quantum coordinate ring of $N_{-}(w) \cap w G_{0}^{\min }$. This isomorphism, called the De Concini-Procesi isomorphism, was proved in DP97, Theorem 3.2 ] under the assumption that $\mathfrak{g}$ is of finite type. We will prove it in the case of arbitrary symmetrizable Kac-Moody cases (Theorem 4.13). We also introduce the dual canonical bases of the quantum unipotent cells (Definition 4.6).

4.1. Quantum unipotent cells. To define the quantum unipotent cells, we use the localizations of $\mathbf{A}_{q}\left[N_{-}(w)\right]$ and $\mathbf{A}_{q}\left[N_{-} \cap X_{w}\right]$. We recall the Ore properties of the unipotent quantum minors. The following is the multiplicative property of the dual canonical bases with respect to the unipotent quantum minors.

Proposition 4.1 ([ $[$ Kim12, Theorem 6.24, Theorem 6.25]). Let $w \in W$.

(1) For $\lambda \in P_{+}$and $b \in \mathscr{B}_{w}(\infty)$, there exists $b^{\prime} \in \mathscr{B}_{w}(\infty)$ such that

$$
q^{-(\lambda, \mathrm{wt} b)}\left[D_{w \lambda, \lambda}\right]\left[G^{\mathrm{up}}(b)\right]=\left[G^{\mathrm{up}}\left(b^{\prime}\right)\right] .
$$

(2) For $\lambda \in P_{+}, \boldsymbol{i} \in I(w)$ and $\boldsymbol{c} \in \mathbb{Z}_{\geq 0}^{\ell(w)}$, we have

$$
q^{-\left(\lambda, \mathrm{wt} b_{-1}(\boldsymbol{c}, \boldsymbol{i})\right)} D_{w \lambda, \lambda} G^{\mathrm{up}}\left(b_{-1}(\boldsymbol{c}, \boldsymbol{i})\right)=G^{\mathrm{up}}\left(b_{-1}\left(\boldsymbol{c}+\boldsymbol{n}^{\lambda}, \boldsymbol{i}\right)\right),
$$

where $\boldsymbol{n}^{\lambda}$ is defined as in Proposition 3.47 .

Proposition 4.1 together with Proposition 3.10 deduces the following (cf. Remark 5.21 below).

Proposition 4.2. Let $w \in W$ and set $\mathcal{D}_{w}:=\left\{q^{m} D_{w \lambda, \lambda} \mid m \in \mathbb{Z}, \lambda \in P_{+}\right\}$. Then the sets $\mathcal{D}_{w}$ and $\left[\mathcal{D}_{w}\right]$ are Ore sets of $\mathbf{A}_{q}\left[N_{-}(w)\right]$ and $\mathbf{A}_{q}\left[N_{-} \cap X_{w}\right]$ respectively consisting of $q$ central elements. More explicitly, for $\lambda, \lambda^{\prime} \in P_{+}$and homogeneous elements $x \in \mathbf{A}_{q}\left[N_{-}(w)\right]$, $y \in \mathbf{A}_{q}\left[N_{-} \cap X_{w}\right]$, we have

$$
\begin{aligned}
q^{-\left(\lambda, w \lambda^{\prime}-\lambda^{\prime}\right)} D_{w \lambda, \lambda} D_{w \lambda^{\prime}, \lambda^{\prime}} & =D_{w\left(\lambda+\lambda^{\prime}\right), \lambda+\lambda^{\prime}} \\
D_{w \lambda, \lambda} x & =q^{(\lambda+w \lambda, \mathrm{wt} x)} x D_{w \lambda, \lambda} \text { in } \mathbf{A}_{q}\left[N_{-}(w)\right], \text { and } \\
{\left[D_{w \lambda, \lambda}\right][y] } & =q^{(\lambda+w \lambda, \mathrm{wt} y)}[y]\left[D_{w \lambda, \lambda}\right] \text { in } \mathbf{A}_{q}\left[N_{-} \cap X_{w}\right] .
\end{aligned}
$$

Using the Proposition 4.2, we obtain the definition of quantum unipotent cells. 
Definition 4.3. For $w \in W$, we set

$$
\begin{aligned}
\mathbf{A}_{q}\left[N_{-}(w) \cap w G_{0}^{\min }\right] & :=\mathbf{A}_{q}\left[N_{-}(w)\right]\left[\mathcal{D}_{w}^{-1}\right], \\
\mathbf{A}_{q}\left[N_{-}^{w}\right] & :=\mathbf{A}_{q}\left[N_{-} \cap X_{w}\right]\left[\left[\mathcal{D}_{w}\right]^{-1}\right] .
\end{aligned}
$$

Those algebras have $Q$-graded algebra structures in an obvious way. The algebra $\mathbf{A}_{q}\left[N_{-}^{w}\right]$ is called a quantum unipotent cell.

Remark 4.4. We note that the notations $\mathbf{A}_{q}\left[N_{-}(w) \cap w G_{0}^{\min }\right]$ and $\mathbf{A}_{q}\left[N_{-}^{w}\right]$ will be justified after proving the existence of the dual canonical bases of that.

4.2. Dual canonical bases of quantum unipotent cells. In this subsection, we define the dual canonical bases of quantum unipotent cells using localization and the "multiplicative property" of the dual canonical bases of $\mathbf{A}_{q}\left[N_{-}^{w}\right]$ and $\mathbf{A}_{q}\left[N_{-}(w) \cap w G_{0}^{\mathrm{min}}\right]$.

Proposition 4.5. Let $w \in W$ and $\boldsymbol{i} \in I(w)$. Then the following hold:

(1) The subset

$$
\left\{q^{(\lambda, \text { wt } b+\lambda-w \lambda)}\left[D_{w \lambda, \lambda}\right]^{-1}\left[G^{\text {up }}(b)\right] \mid \lambda \in P_{+}, b \in \mathscr{B}_{w}(\infty)\right\}
$$

of $\mathbf{A}_{q}\left[N_{-}^{w}\right]$ forms a $\mathbb{Q}(q)$-basis of $\mathbf{A}_{q}\left[N_{-}^{w}\right]$.

(2) The subset

$$
\left\{q^{\left(\lambda, \mathrm{wt} b_{-1}(\boldsymbol{c}, \boldsymbol{i})+\lambda-w \lambda\right)} D_{w \lambda, \lambda}^{-1} G^{\mathrm{up}}\left(b_{-1}(\boldsymbol{c}, \boldsymbol{i})\right) \mid \lambda \in P_{+}, \boldsymbol{c} \in \mathbb{Z}_{\geq 0}^{\ell(w)}\right\}
$$

of $\mathbf{A}_{q}\left[N_{-}(w) \cap w G_{0}^{\mathrm{min}}\right]$ forms a $\mathbb{Q}(q)$-basis of $\mathbf{A}_{q}\left[N_{-}(w) \cap w G_{0}^{\mathrm{min}}\right]$.

Proof. We prove only (1). The assertion (2) is proved in the same manner. The given subset obviously spans the $\mathbb{Q}(q)$-vector space $\mathbf{A}_{q}\left[N_{-}^{w}\right]$. Hence it remains to show that this set is a linearly independent set. For $(\lambda, b),\left(\lambda^{\prime}, b^{\prime}\right) \in P_{+} \times \mathscr{B}_{w}(\infty)$, write $(\lambda, b) \sim\left(\lambda^{\prime}, b^{\prime}\right)$ if and only if $q^{(\lambda, \mathrm{wt} b+\lambda-w \lambda)}\left[D_{w \lambda, \lambda}\right]^{-1}\left[G^{\mathrm{up}}(b)\right]=q^{\left(\lambda^{\prime}, \mathrm{wt} b^{\prime}+\lambda^{\prime}-w \lambda^{\prime}\right)}\left[D_{w \lambda^{\prime}, \lambda^{\prime}}\right]^{-1}\left[G^{\mathrm{up}}\left(b^{\prime}\right)\right]$. The relation $\sim$ is clearly an equivalence relation, and we take a complete set $F$ of coset representatives of $\left(P_{+} \times \mathscr{B}_{w}(\infty)\right) / \sim$.

Suppose that there exists a finite subset $F^{\prime} \subset F$ and $a_{\lambda, b} \in \mathbb{Q}(q)\left((\lambda, b) \in F^{\prime}\right)$ such that $\sum_{(\lambda, b) \in F^{\prime}} q^{(\lambda, \mathrm{wt} b+\lambda-w \lambda)} a_{\lambda, b}\left[D_{w \lambda, \lambda}\right]^{-1}\left[G^{\text {up }}(b)\right]=0$. There exists $\lambda_{0} \in P_{+}$such that $\lambda_{0}-$ $\lambda \in P_{+}$for all $\lambda \in P_{+}$such that $(\lambda, b) \in F^{\prime}$ for some $b \in \mathscr{B}_{w}(\infty)$. Now the equality $\sum_{(\lambda, b) \in F^{\prime}} q^{(\lambda, \mathrm{wt} b+\lambda-w \lambda)} a_{\lambda, b}\left[D_{w \lambda, \lambda}\right]^{-1}\left[G^{\mathrm{up}}(b)\right]=0$ is equivalent to the equality

$$
\left[D_{w \lambda_{0}, \lambda_{0}}\right]\left(\sum_{(\lambda, b) \in F^{\prime}} q^{(\lambda, \mathrm{wt} b+\lambda-w \lambda)} a_{\lambda, b}\left[D_{w \lambda, \lambda}\right]^{-1}\left[G^{\mathrm{up}}(b)\right]\right)=0 .
$$

By Proposition 4.2 and Proposition 4.1, for $(\lambda, b) \in F^{\prime}$, we have

$$
\begin{aligned}
{\left[D_{w \lambda_{0}, \lambda_{0}}\right]\left(q^{(\lambda, \mathrm{wt} b+\lambda-w \lambda)}\left[D_{w \lambda, \lambda}\right]^{-1}\left[G^{\mathrm{up}}(b)\right]\right) } & =q^{-\left(\lambda_{0}-\lambda, w \lambda-\lambda\right)+(\lambda, \operatorname{wt} b+\lambda-w \lambda)}\left[D_{w\left(\lambda_{0}-\lambda\right),\left(\lambda_{0}-\lambda\right)}\right]\left[G^{\mathrm{up}}(b)\right] \\
& =q^{\left(\lambda_{0}, \mathrm{wt} b+\lambda-w \lambda\right)}\left[G^{\mathrm{up}}\left(b^{\left(\lambda_{0}-\lambda\right)}\right)\right]
\end{aligned}
$$

for some $b^{\left(\lambda_{0}-\lambda\right)} \in \mathscr{B}_{w}(\infty)$. Note that wt $b+\lambda-w \lambda=\operatorname{wt} b^{\left(\lambda_{0}-\lambda\right)}-\operatorname{wt} D_{w \lambda_{0}, \lambda_{0}}$. Therefore if $b^{\left(\lambda_{0}-\lambda\right)}=\left(b^{\prime}\right)^{\left(\lambda_{0}-\lambda^{\prime}\right)}$ for $(\lambda, b),\left(\lambda^{\prime}, b^{\prime}\right) \in F^{\prime}$ then we have the equality

$\left[D_{w \lambda_{0}, \lambda_{0}}\right]\left(q^{(\lambda, \mathrm{wt} b+\lambda-w \lambda)}\left[D_{w \lambda, \lambda}\right]^{-1}\left[G^{\mathrm{up}}(b)\right]\right)=\left[D_{w \lambda_{0}, \lambda_{0}}\right]\left(q^{\left(\lambda^{\prime}, \mathrm{wt} b^{\prime}+\lambda^{\prime}-w \lambda^{\prime}\right)}\left[D_{w \lambda^{\prime}, \lambda^{\prime}}\right]^{-1}\left[G^{\mathrm{up}}\left(b^{\prime}\right)\right]\right)$, 
hence $(\lambda, b)=\left(\lambda^{\prime}, b^{\prime}\right)$. Thus (4.1) implies $a_{\lambda, b}=0$ for all $(\lambda, b) \in F^{\prime}$. This completes the proof.

Definition 4.6. Let $w \in W$. We call

$$
\begin{aligned}
\widetilde{\mathbf{B}}^{\mathrm{up}, w} & :=\left\{q^{(\lambda, \mathrm{wt} b+\lambda-w \lambda)}\left[D_{w \lambda, \lambda}\right]^{-1}\left[G^{\mathrm{up}}(b)\right] \mid \lambda \in P_{+}, b \in \mathscr{B}_{w}(\infty)\right\}, \text { and } \\
\widetilde{\mathbf{B}}^{\mathrm{up}}(w) & :=\left\{q^{\left(\lambda, \mathrm{wt} b_{-1}(\boldsymbol{c}, \boldsymbol{i})+\lambda-w \lambda\right)} D_{w \lambda, \lambda}^{-1} G^{\mathrm{up}}\left(b_{-1}(\boldsymbol{c}, \boldsymbol{i})\right) \mid \lambda \in P_{+}, \boldsymbol{c} \in \mathbb{Z}_{\geq 0}^{\ell(w)}\right\}
\end{aligned}
$$

the dual canonical bases of $\mathbf{A}_{q}\left[N_{-}^{w}\right]$ and $\mathbf{A}_{q}\left[N_{-}(w) \cap w G_{0}^{\mathrm{min}}\right]$, respectively.

For $\lambda \in P$, there exist $\lambda_{1}, \lambda_{2} \in P_{+}$such that $\lambda=-\lambda_{1}+\lambda_{2}$. Set

$$
D_{w, \lambda}:=q^{\left(\lambda_{1}, w \lambda-\lambda\right)} D_{w \lambda_{1}, \lambda_{1}}^{-1} D_{w \lambda_{2}, \lambda_{2}} \in \widetilde{\mathbf{B}}^{\mathrm{up}}(w) .
$$

Then $D_{w, \lambda}$ does not depend on the choice of $\lambda_{1}, \lambda_{2} \in P_{+}$by Proposition 4.5. Note that wt $D_{w, \lambda}=w \lambda-\lambda$.

The following is straightforwardly proved by Proposition 4.2 .

Proposition 4.7. Let $w \in W$ and $\lambda, \lambda^{\prime} \in P_{+}$. Then the following hold:

(1) $D_{w, \lambda}=q^{\left(\lambda, w \lambda_{1}-\lambda_{1}\right)} D_{w \lambda_{2}, \lambda_{2}} D_{w \lambda_{1}, \lambda_{1}}^{-1}$ for $\lambda_{1}, \lambda_{2} \in P_{+}$with $\lambda=-\lambda_{1}+\lambda_{2}$.

(2) $D_{w, \lambda} D_{w, \lambda^{\prime}}=q^{\left(\lambda, w \lambda^{\prime}-\lambda^{\prime}\right)} D_{w, \lambda+\lambda^{\prime}}$. In particular, $D_{w, \lambda}^{-1}=q^{(\lambda, w \lambda-\lambda)} D_{w,-\lambda}$.

(3) $D_{w, \lambda} x=q^{(\lambda+w \lambda, w t x)} x D_{w, \lambda}$ for $\lambda \in P_{+}$and a homogeneous element $x \in \mathbf{A}_{q}\left[N_{-}(w) \cap\right.$ $\left.w G_{0}^{\min }\right]$.

Remark 4.8. By using Proposition 4.1 (2), we can parametrize explicitly the elements of $\widetilde{\mathbf{B}}^{\text {up }}(w)$. Fix $\boldsymbol{i}=\left(i_{1}, \ldots, i_{\ell}\right) \in I(w)$. An element $\boldsymbol{c} \in \mathbb{Z}_{>0}^{\ell}$ is said to have gaps if $\min \left\{c_{k} \mid i_{k}=\right.$ $i\}=0$ for all $i \in I$. Then, by Propositions 4.1 (2) and 4.5 (2), we obtain the non-overlapping parametrization of the elements of $\widetilde{\mathbf{B}}^{\text {up }}(w)$ as follows:

$$
\widetilde{\mathbf{B}}^{\mathrm{up}}(w)=\left\{q^{-\left(\lambda, \mathrm{wt} b_{-1}(\boldsymbol{c}, \boldsymbol{i})\right)} D_{w, \lambda} G^{\mathrm{up}}\left(b_{-1}(\boldsymbol{c}, \boldsymbol{i})\right) \mid \lambda \in P, \boldsymbol{c} \in \mathbb{Z}_{\geq 0}^{\ell} \text { has gaps }\right\} .
$$

We define the dual bar involutions on $\mathbf{A}_{q}\left[N_{-}^{w}\right]$ and $\mathbf{A}_{q}\left[N_{-}(w) \cap w G_{0}^{\min }\right]$, which are useful when we study the dual canonical bases.

Proposition 4.9. The following hold:

(1) The twisted dual bar involution $\sigma^{\prime}$ induces $\mathbb{Q}$-algebra anti-involutions $\mathbf{A}_{q}\left[N_{-} \cap X_{w}\right] \rightarrow$ $\mathbf{A}_{q}\left[N_{-} \cap X_{w}\right]$ and $\mathbf{A}_{q}\left[N_{-}(w)\right] \rightarrow \mathbf{A}_{q}\left[N_{-}(w)\right]$. See Definition 3.11 for the definition of $\sigma^{\prime}$. Moreover these maps are extended to $\mathbb{Q}$-algebra anti-involutions $\sigma^{\prime}: \mathbf{A}_{q}\left[N_{-}^{w}\right] \rightarrow \mathbf{A}_{q}\left[N_{-}^{w}\right]$ and $\sigma^{\prime}: \mathbf{A}_{q}\left[N_{-}(w) \cap w G_{0}^{\min }\right] \rightarrow \mathbf{A}_{q}\left[N_{-}(w) \cap w G_{0}^{\min }\right]$.

(2) Define a $\mathbb{Q}(q)$-linear isomorphism $c_{\mathrm{tw}}: \mathbf{A}_{q}\left[N_{-}^{w}\right] \rightarrow \mathbf{A}_{q}\left[N_{-}^{w}\right]$ (resp. $\mathbf{A}_{q}\left[N_{-}(w) \cap w G_{0}^{\mathrm{min}}\right] \rightarrow$ $\left.\mathbf{A}_{q}\left[N_{-}(w) \cap w G_{0}^{\mathrm{min}}\right]\right)$ by

$$
x \mapsto q^{(\mathrm{wt} x, \mathrm{wt} x) / 2-(\mathrm{wt} x, \rho)} x
$$

for every homogeneous element $x \in \mathbf{A}_{q}\left[N_{-}^{w}\right]$ (resp. $x \in \mathbf{A}_{q}\left[N_{-}(w) \cap w G_{0}^{\min }\right]$ ).

Set $\sigma:=c_{\mathrm{tw}} \circ \sigma^{\prime}$. Then for homogeneous elements $x, y \in \mathbf{A}_{q}\left[N_{-}^{w}\right]\left(\operatorname{resp} . \mathbf{A}_{q}\left[N_{-}(w) \cap w G_{0}^{\mathrm{min}}\right]\right)$ we have

$$
\sigma(x y)=q^{(\text {wt } x, \text { wt } y)} \sigma(y) \sigma(x) .
$$

Moreover the elements of the dual canonical bases $\widetilde{\mathbf{B}}^{\text {up }, w}$ and $\widetilde{\mathbf{B}}^{\text {up }}(w)$ are fixed by $\sigma$. 
Definition 4.10. The $\mathbb{Q}$-linear isomorphisms $\sigma$ and $\sigma^{\prime}: \mathbf{A}_{q}\left[N_{-}^{w}\right] \rightarrow \mathbf{A}_{q}\left[N_{-}^{w}\right], \mathbf{A}_{q}\left[N_{-}(w) \cap\right.$ $\left.w G_{0}^{\text {min }}\right] \rightarrow \mathbf{A}_{q}\left[N_{-}(w) \cap w G_{0}^{\text {min }}\right]$ defined in Proposition 4.9 will be also called the dual bar involution and the twisted dual bar involution, respectively.

Proof of Proposition 4.9. Recall that $\sigma^{\prime}\left(G^{\mathrm{up}}(b)\right)=q^{-(\mathrm{wt} b, \mathrm{wt} b) / 2+(\mathrm{wt} b, \rho)} G^{\mathrm{up}}(b)$ for all $b \in$ $\mathscr{B}(\infty)$. See Remark 3.12 . Hence (1) follows from the compatibility of the algebras $\mathbf{A}_{q}\left[N_{-} \cap\right.$ $\left.X_{w}\right], \mathbf{A}_{q}\left[N_{-}(w)\right]$ and the dual canonical basis (Definition 3.28, Definition 3.37), and the universality of localization [GW04, Proposition 6.3]. A direct calculation immediately shows the equality 4.2, For $\lambda \in P_{+}$, we have

$$
\begin{aligned}
1 & =\sigma\left(D_{w \lambda, \lambda} D_{w \lambda, \lambda}^{-1}\right) \\
& =q^{-(w \lambda-\lambda, w \lambda-\lambda)} \sigma\left(D_{w \lambda, \lambda}^{-1}\right) \sigma\left(D_{w \lambda, \lambda}\right) \\
& =q^{2(\lambda, w \lambda-\lambda)} \sigma\left(D_{w \lambda, \lambda}^{-1}\right) D_{w \lambda, \lambda}
\end{aligned}
$$

in $\mathbf{A}_{q}\left[N_{-}(w) \cap w G_{0}^{\min }\right]$. Hence

$$
\sigma\left(D_{w \lambda, \lambda}^{-1}\right)=q^{-2(\lambda, w \lambda-\lambda)} D_{w \lambda, \lambda}^{-1}
$$

Let $b \in \mathscr{B}_{w}(\infty)$. Then, by Proposition 4.2 and the equality above, we have

$$
\begin{aligned}
& \sigma\left(q^{(\lambda, \mathrm{wt} b+\lambda-w \lambda)}\left[D_{w \lambda, \lambda}\right]^{-1}\left[G^{\mathrm{up}}(b)\right]\right) \\
& =q^{-(\lambda, \mathrm{wt} b+\lambda-w \lambda)+(\lambda-w \lambda, \mathrm{wt} b)} \sigma\left(\left[G^{\mathrm{up}}(b)\right]\right) \sigma\left(\left[D_{w \lambda, \lambda}\right]^{-1}\right) \\
& =q^{-(\lambda, \mathrm{wt} b+\lambda-w \lambda)+(\lambda-w \lambda, \mathrm{wt} b)-2(\lambda, w \lambda-\lambda)}\left[G^{\mathrm{up}}(b)\right]\left[D_{w \lambda, \lambda}\right]^{-1} \\
& =q^{-(\lambda, \mathrm{wt} b+\lambda-w \lambda)+(\lambda-w \lambda, \mathrm{wt} b)-2(\lambda, w \lambda-\lambda)+(\lambda+w \lambda, \mathrm{wt} b)}\left[D_{w \lambda, \lambda}\right]^{-1}\left[G^{\mathrm{up}}(b)\right] \\
& =q^{(\lambda, \mathrm{wt} b+\lambda-w \lambda)}\left[D_{w \lambda, \lambda}\right]^{-1}\left[G^{\mathrm{up}}(b)\right] .
\end{aligned}
$$

This proves the dual bar invariance property for $\widetilde{\mathbf{B}}^{\text {up }, w}$. The assertion for $\tilde{\mathbf{B}}^{\text {up }}(w)$ is proved in the same manner.

As a corollary of the existence of the dual canonical bases of $\mathbf{A}_{q}\left[N_{-}(w) \cap w G_{0}^{\min }\right]$ and $\mathbf{A}_{q}\left[N_{-}^{w}\right]$, we have the following specialization theorem.

Corollary 4.11. Let $w \in W$.

(1) Set $\mathbf{A}_{\mathbb{Q}\left[q^{ \pm 1}\right]}\left[N_{-}(w) \cap w G_{0}^{\min }\right]$ to be the free $\mathcal{A}$-module spanned by $\widetilde{\mathbf{B}}^{\mathrm{up}}(w)$. Then it is a $\mathcal{A}$-subalgebra of $\mathbf{A}_{q}\left[N_{-}(w) \cap w G_{0}^{\min }\right]$ and we have an isomorphism

$$
\mathbf{A}_{\mathbb{Q}\left[q^{ \pm 1}\right]}\left[N_{-}(w) \cap w G_{0}^{\min }\right] \otimes_{\mathcal{A}} \mathbb{C} \simeq \mathbb{C}\left[N_{-}(w) \cap w G_{0}^{\min }\right]
$$

as $\mathbb{C}$-algebras.

(2) Set $\mathbf{A}_{\mathbb{Q}\left[q^{ \pm 1}\right]}\left[N_{-}^{w}\right]$ to be the free $\mathcal{A}$-module spanned by $\widetilde{\mathbf{B}}^{\mathrm{up}, w}$. Then it is a $\mathcal{A}$-subalgebra of $\mathbf{A}_{q}\left[N_{-}^{w}\right]$ and we have an isomorphism

$$
\mathbf{A}_{\mathbb{Q}\left[q^{ \pm 1}\right]}\left[N_{-}^{w}\right] \otimes_{\mathcal{A}} \mathbb{C} \simeq \mathbb{C}\left[N_{-}^{w}\right]
$$

as $\mathbb{C}$-algebras. 
4.3. De Concini-Procesi isomorphisms. In this subsection, we give a proof of the De Concini-Procesi isomorphism between $\mathbf{A}_{q}\left[N_{-}(w)\right]$ and $\mathbf{A}_{q}\left[N_{-} \cap X_{w}\right]$ for general symmetrizable Kac-Moody Lie algebras, by using theory of canonical bases and specialization. We should remark that the original proof in [DP97] uses the downward induction on the length of elements of the Weyl group $W$ from the longest element, which exists only in finite type cases.

Proposition 4.12 ([Kim12, Theorem 5.13]). Let $w \in W$. Define $\iota_{w}: \mathbf{A}_{q}\left[N_{-}(w)\right] \rightarrow \mathbf{A}_{q}\left[N_{-} \cap\right.$ $\left.X_{w}\right]$ as a $\mathbb{Q}(q)$-algebra homomorphism induced from the canonical projection $\mathbf{U}_{q}^{-} \rightarrow \mathbf{A}_{q}\left[N_{-} \cap\right.$ $X_{w}$ ]. Recall Definition 3.26 and 3.37. Then $\iota_{w}$ is injective, or equivalently, $*\left(\mathscr{B}\left(\mathbf{U}_{q}^{-}(w)\right)\right) \subset$ $\mathscr{B}_{w}(\infty)$.

Theorem 4.13 (The De Concini-Procesi isomorphism). Let $w \in W$. Then $\iota_{w}$ induces an isomorphism;

$$
\iota_{w}: \mathbf{A}_{q}\left[N_{-}(w) \cap w G_{0}^{\min }\right] \stackrel{\sim}{\rightarrow} \mathbf{A}_{q}\left[N_{-}^{w}\right] .
$$

Proof. The map $\iota_{w}$ in Proposition 4.12 induces an injective algebra homomorphism $\iota_{w}: \mathbf{A}_{q}\left[N_{-}(w)\right] \rightarrow$ $\mathbf{A}_{q}\left[N_{-}^{w}\right]$. Since this map sends $D_{w \lambda, \lambda}$ to $\left[D_{w \lambda, \lambda}\right]$ for $\lambda \in P_{+}$, it is extended to the injective algebra homomorphism

$$
\iota_{w}: \mathbf{A}_{q}\left[N_{-}(w) \cap w G_{0}^{\min }\right]=\mathbf{A}_{q}\left[N_{-}(w)\right]\left[\mathcal{D}_{w}^{-1}\right] \rightarrow \mathbf{A}_{q}\left[N_{-}^{w}\right]
$$

by the universality of localization. It follows immediately from the definition of dual canonical bases and Proposition 4.12 that $\iota_{w}$ induces an injective map from $\widetilde{\mathbf{B}}^{\text {up }}(w)$ to $\widetilde{\mathbf{B}}^{\text {up }, w}$. Therefore the map (4.3) is an isomorphism if and only if the (well-defined) map

$$
\left.\iota_{w}\right|_{q=1}: \mathbf{A}_{\mathbb{Q}\left[q^{ \pm 1}\right]}\left[N_{-}(w) \cap w G_{0}^{\mathrm{min}}\right] \otimes_{\mathcal{A}} \mathbb{C} \rightarrow \mathbf{A}_{\mathbb{Q}\left[q^{ \pm 1}\right]}\left[N_{-}^{w}\right] \otimes_{\mathcal{A}} \mathbb{C}
$$

is an isomorphism. Through the isomorphisms in Corollary 4.11, the map $\left.\iota_{w}\right|_{q=1}$ coincides with the map in Corollary 2.22 by definition of $\iota_{w}$; hence it is an isomorphism. This completes the proof.

\section{Quantum Twist ISOMORPhisms}

In this section, we construct the quantum twist isomorphisms between $\mathbf{A}_{q}\left[N_{-}(w) \cap w G_{0}^{\min }\right]$ and $\mathbf{A}_{q}\left[N_{-}^{w}\right]$ (see Theorem [5.19) and define the quantum twist automorphisms on $\mathbf{A}_{q}\left[N_{-}^{w}\right]$ as a composite of the quantum twist isomorphism and the De Concini-Procesi isomorphism.

5.1. Quantized coordinate algebras. In this subsection, we give a brief review on the quantized coordinate rings. For more details, see [Jos95, Chapter 9, 10].

Definition 5.1. Let $M$ be a $\mathbf{U}_{q}$-module. For $f \in M^{*}:=\operatorname{Hom}_{\mathbb{Q}(q)}(M, \mathbb{Q}(q))$ and $u \in M$, define a $\mathbb{Q}(q)$-linear map $c_{f, u}^{M} \in \mathbf{U}_{q}^{*}$ given by

$$
x \mapsto\langle f, x . u\rangle
$$

for $x \in \mathbf{U}_{q}$. When $M=V(\lambda)\left(\lambda \in P_{+}\right)$, we abbreviate $c_{f, u}^{V(\lambda)}$ to $c_{f, u}^{\lambda}$. For $w, w^{\prime} \in W$ and $\lambda \in P_{+}$, we write

$$
c_{w \lambda, w^{\prime} \lambda}^{\lambda}:=c_{f_{w \lambda}, u_{w^{\prime} \lambda}}^{\lambda},
$$

here $f_{w \lambda} \in V(\lambda)^{*}$ is defined by $u \mapsto\left(u_{w \lambda}, u\right)_{\lambda}^{\varphi}$. 
Definition 5.2. Let $M$ be a $\mathbf{U}_{q}$-module. For $\mu \in P$, we set

$$
M_{\mu}:=\left\{m \in M \mid q^{h} \cdot m=q^{\langle\mu, h\rangle} m \text { for all } h \in P^{*}\right\} .
$$

For a $\mathbf{U}_{q}$-module $M=\bigoplus_{\mu \in P} M_{\mu}$ with weight space decomposition, we write its graded dual $\bigoplus_{\mu \in P} M_{\mu}^{*}$ as $M^{\star}$. Note that $M^{\star}$ is a right $\mathbf{U}_{q^{-}}$-module. For $\lambda \in P_{+}, V(\lambda)^{\star}$ is an integrable highest weight right $\mathbf{U}_{q^{-}}$module with highest weight $\lambda$. For $u \in V(\lambda)$, define $u^{*} \in V(\lambda)^{\star}$ by $u^{\prime} \mapsto\left(u, u^{\prime}\right)_{\lambda}^{\varphi}$. Then we have $V(\lambda)^{\star}=\left\{u^{*} \mid u \in V(\lambda)\right\}$ since the bilinear form $(,)_{\lambda}^{\varphi}$ is non-degenerate. Note that $f_{w \lambda}=u_{w \lambda}^{*}$ for $w \in W$.

Let $\mathbf{R}_{q}$ be the $\mathbb{Q}(q)$-vector subspace of $\mathbf{U}_{q}^{*}$ spanned by the elements

$$
\left\{c_{f, u}^{\lambda} \mid f \in V(\lambda)^{\star}, u \in V(\lambda) \text { and } \lambda \in P_{+}\right\} \text {. }
$$

Henceforth, we consider the algebra structure of $\mathbf{U}_{q}^{*}$ induced from the coalgebra structure of $\mathbf{U}_{q}$.

Proposition 5.3 ([Kas93b, Definition 7.2.1, Proposition 7.2.2]). The subspace $\mathbf{R}_{q}$ is a subalgebra of $\mathbf{U}_{q}^{*}$, which is isomorphic to $\bigoplus_{\lambda \in P_{+}} V(\lambda)^{\star} \otimes V(\lambda)$ as a $\mathbf{U}_{q^{-}}$-bimodule.

The $\mathbb{Q}(q)$-algebra $\mathbf{R}_{q}$ is called the quantized coordinate algebra associated with $\mathbf{U}_{q}$.

Definition 5.4. Let $v, w \in W$ and $\lambda \in P_{+}$. Set

$$
\begin{aligned}
& \mathbf{R}_{q}^{w(+)}(\lambda):=\left\{c_{f, u_{w \lambda}}^{\lambda} \mid f \in V(\lambda)^{\star}\right\}, \mathbf{R}_{q}^{w(+)}:=\sum_{\lambda^{\prime} \in P_{+}} \mathbf{R}_{q}^{w(+)}\left(\lambda^{\prime}\right) \subset \mathbf{R}_{q}, \\
& \mathbf{Q}_{v}^{w(+)}(\lambda):=\left\{c_{f, u_{w \lambda}}^{\lambda} \mid f \in V(\lambda)^{\star},\left\langle f, \mathbf{U}_{q}^{+} . u_{v \lambda}\right\rangle=0\right\}, \mathbf{Q}_{v}^{w(+)}:=\sum_{\lambda^{\prime} \in P_{+}} \mathbf{Q}_{v}^{w(+)}\left(\lambda^{\prime}\right) \subset \mathbf{R}_{q} .
\end{aligned}
$$

When $w=e$, we write $\mathbf{R}_{q}^{e(+)}$ (resp. $\mathbf{Q}_{v}^{e(+)}$ ) as $\mathbf{R}_{q}^{+}$(resp. $\mathbf{Q}_{u}^{+}$). It is easy to show that, for all $w \in W, \mathbf{R}_{q}^{w(+)}$ is a subalgebra of $\mathbf{R}_{q}$, and isomorphic to $\mathbf{R}_{q}^{+}$as algebras via $c_{f, u_{w \lambda}}^{\lambda} \mapsto c_{f, u_{\lambda}}^{\lambda}$. See, for example, [Tan17, Chapter 3]. Moreover, for all $v, w \in W, \mathbf{Q}_{v}^{w(+)}$ is a two-sided ideal of $\mathbf{R}_{q}^{w(+)}$, and the above isomorphism induces an isomorphism from $\mathbf{R}_{q}^{w(+)} / \mathbf{Q}_{v}^{w(+)}$ to $\mathbf{R}_{q}^{+} / \mathbf{Q}_{v}^{+}$.

5.2. Other descriptions of quantum unipotent subgroups and quantum closed unipotent cells. In this subsection, we describe the algebras, quantum unipotent subgroups and quantum unipotent cells, by using the quantized coordinate algebra $\mathbf{R}_{q}$. The following descriptions are essentially shown in [Jos95, 9.1.7], Yak10, Theorem 3.7]. However, we restate them emphasizing the terms of dual canonical bases. Actually, we can now prove each statement immediately.

Notation 5.5. Let $v, w \in W$. By abuse of notation, we describe the canonical projection $\mathbf{R}_{q}^{w(+)} \rightarrow \mathbf{R}_{q}^{w(+)} / \mathbf{Q}_{v}^{w(+)}$ as $c \mapsto[c]$.

Definition 5.6. As a bridge between quantized enveloping algebras and quantized coordinate algebras, we consider the following two linear maps:

$$
\begin{aligned}
\Phi: \check{\mathbf{U}}_{\bar{q}}^{\leq 0} \rightarrow\left(\mathbf{U}_{\bar{q}}^{\leq 0}\right)^{*}, y_{1} \mapsto\left(y_{2} \mapsto\left(\psi\left(y_{1}\right), y_{2}\right)_{D}\right), \\
\Phi^{+}: \check{\mathbf{U}}_{\bar{q}}^{\geq 0} \rightarrow\left(\mathbf{U}_{\bar{q}}^{\geq 0}\right)^{*}, x_{1} \mapsto\left(x_{2} \mapsto\left(x_{1}, \psi\left(x_{2}\right)\right)_{D}\right) .
\end{aligned}
$$

By the properties of the Drinfeld pairing $(,)_{D}, \Phi$ is an injective algebra homomorphism and $\Phi^{+}$is an injective algebra anti-homomorphism. 
Definition 5.7. Let $\lambda \in P_{+}$. Set

$$
\mathbf{U}_{q}^{-}(\lambda):=j_{\lambda}(V(\lambda))=\sum_{b \in \mathscr{B}(\lambda)} \mathbb{Q}(q) G^{\mathrm{up}}\left(\bar{\jmath}_{\lambda}(b)\right) .
$$

The following propositions follow from the non-degeneracy of the Drinfeld pairing, Lemma 3.8 and Proposition 3.46 .

Proposition 5.8. The restriction $\operatorname{map} \mathbf{U}_{q}^{*} \rightarrow\left(\mathbf{U}_{\bar{q}}^{\leq 0}\right)^{*}$ induces the injective algebra homomorphism $r_{\leq 0}: \mathbf{R}_{q}^{+} \rightarrow\left(\mathbf{U}_{q}^{\leq 0}\right)^{*}$, and $\operatorname{Im} r_{\leq 0} \subset \operatorname{Im} \Phi$. Moreover the well-defined $\mathbb{Q}(q)$-algebra homomorphism $\mathbf{R}_{q}^{+} \rightarrow \check{\mathbf{U}}_{q}^{\leq 0}, c \mapsto\left(\Phi^{-1} \circ r_{\leq 0}\right)(c)$ induces the $\mathbb{Q}(q)$-algebra isomorphism $\mathcal{I}: \mathbf{R}_{q}^{+} \rightarrow \sum_{\lambda \in P_{+}} \mathbf{U}_{q}^{-}(\lambda) q^{-\lambda}$.

Proposition 5.9. For $\lambda \in P_{+}$and $b \in \mathscr{B}(\lambda)$, we have

$$
\mathcal{I}\left(c_{G_{\lambda}^{\mathrm{up}}(b)^{*}, u_{\lambda}}^{\mathrm{u}}\right)=G^{\mathrm{up}}\left(\bar{\jmath}_{\lambda}(b)\right) q^{-\lambda}=D_{G_{\lambda}^{\mathrm{up}}(b), u_{\lambda}} q^{-\lambda} .
$$

In particular, we have

$$
\mathcal{I}\left(\mathbf{Q}_{w}^{+}(\lambda)\right)=\sum_{b \in \mathscr{B}(\lambda) \backslash \mathscr{B}_{w}(\lambda)} \mathbb{Q}(q) G^{\text {up }}\left(\bar{\jmath}_{\lambda}(b)\right) q^{-\lambda} .
$$

Definition 5.10. An element $z$ of $\mathbf{R}_{q}^{+}$(resp. $\mathbf{R}_{q}^{+} / \mathbf{Q}_{w}^{+}$) is said to be $q$-central if, for every weight vector $f \in V(\lambda)^{\star}$ and $\lambda \in P_{+}$, there exists $l \in \mathbb{Z}$ such that

$$
z c_{f, u_{\lambda}}^{\lambda}=q^{l} c_{f, u_{\lambda}}^{\lambda} z \quad\left(\text { resp. } z\left[c_{f, u_{\lambda}}^{\lambda}\right]=q^{l}\left[c_{f, u_{\lambda}}^{\lambda}\right] z\right) .
$$

Corollary 5.11. The set $\left\{c_{\lambda, \lambda}^{\lambda}\right\}_{\lambda \in P_{+}}$is an Ore set in $\mathbf{R}_{q}^{+}$consisting of q-central elements. In particular, $\mathcal{S}:=\left\{\left[c_{\lambda, \lambda}^{\lambda}\right]\right\}_{\lambda \in P_{+}}$is an Ore set in $\mathbf{R}_{q}^{+} / \mathbf{Q}_{w}^{+}$consisting of q-central elements.

By Corollary 5.11, we can consider the algebra $\left(\mathbf{R}_{q}^{+} / \mathbf{Q}_{w}^{+}\right)\left[\mathcal{S}^{-1}\right]$. Proposition 5.8 and 5.9 together with Remark 3.36 immediately imply the following proposition. This gives the description of $\mathbf{A}_{q}\left[N_{-} \cap X_{w}\right]$ in terms of the quantized coordinate algebra $\mathbf{R}_{q}$. This kind of description appears in [Jos95, 9.1.7].

Proposition 5.12. Let $w \in W$. Set $\mathbf{A}_{q}\left[N_{-} \cap X_{w}\right]^{\text {ex }}:=\check{\mathbf{U}}_{\bar{q}}^{\leq 0} /\left(\mathbf{U}_{w}^{-}\right)^{\perp} \check{\mathbf{U}}_{q}^{0}$. Note that $\left(\mathbf{U}_{w}^{-}\right)^{\perp} \check{\mathbf{U}}_{q}^{0}$ is a two-sided ideal of $\check{\mathbf{U}}_{q}^{\leq 0}$. Then the $\mathbb{Q}(q)$-algebra isomorphism $\mathcal{I}: \mathbf{R}_{q}^{+} \rightarrow \sum_{\lambda \in P_{+}} \mathbf{U}_{q}^{-}(\lambda) q^{-\lambda}$ induces the $\mathbb{Q}(q)$-algebra isomorphism

$$
\mathcal{I}_{w}:\left(\mathbf{R}_{q}^{+} / \mathbf{Q}_{w}^{+}\right)\left[\mathcal{S}^{-1}\right] \rightarrow \mathbf{A}_{q}\left[N_{-} \cap X_{w}\right]^{\mathrm{ex}} .
$$

Moreover the $\mathbb{Q}(q)$-algebra $\sum_{\lambda \in P_{+}}\left(\mathbf{R}_{q}^{+}(\lambda) / \mathbf{Q}_{w}^{+}\right)\left[c_{\lambda, \lambda}^{\lambda}\right]^{-1}\left(\subset\left(\mathbf{R}_{q}^{+} / \mathbf{Q}_{w}^{+}\right)\left[\mathcal{S}^{-1}\right]\right)$ is isomorphic to $\mathbf{A}_{q}\left[N_{-} \cap X_{w}\right]$.

Next, we study the quantum unipotent subgroups via the quantized coordinate rings following Joseph and Yakimov. We consider the algebra $\mathbf{R}_{q}^{w(+)} / \mathbf{Q}_{w}^{w(+)}$, which is isomorphic to $\mathbf{R}_{q}^{+} / \mathbf{Q}_{w}^{+}$. See Definition 5.4.

Definition 5.13. Let $w \in W$ and $\lambda \in P_{+}$. Set

$$
\mathbf{U}_{q}^{+}(w, \lambda):=\left(j_{w \lambda}^{\vee}\left(V(\lambda) / V_{w}(\lambda)^{\perp}\right)\right)^{\vee}=\sum_{b \in \mathscr{B}_{w}(\lambda)} \mathbb{Q}(q) G^{\text {up }}\left(\bar{\jmath}_{w \lambda}^{\vee}(b)\right)^{\vee} .
$$

The following proposition follows again from the non-degeneracy of the Drinfeld pairing, the equality (3.2), Lemma 3.8, Proposition 3.19 and Proposition 3.46. 
Proposition 5.14. Let $w \in W$. The restriction map $\mathbf{U}_{q}^{*} \rightarrow\left(\mathbf{U}_{\bar{q}}^{\geq 0}\right)^{*}$ induces the algebra homomorphism $r_{\geq 0}^{w}: \mathbf{R}_{q}^{w(+)} \rightarrow\left(\mathbf{U}_{\bar{q}}^{\geq 0}\right)^{*}$, and it satisfies $\operatorname{Ker}\left(r_{\geq 0}^{w}\right)=\mathbf{Q}_{w}^{w(+)}$ and $\operatorname{Im} r_{\geq 0}^{w} \subset \operatorname{Im} \Phi^{+}$. Hence $r_{\geq 0}^{w}$ induces the $\mathbb{Q}(q)$-algebra isomorphism $\bar{r}_{\geq 0}^{w}: \mathbf{R}_{q}^{w(+)} / \mathbf{Q}_{w}^{w(+)} \rightarrow \operatorname{Im} r_{\geq 0}^{w}$. Moreover we have a well-defined algebra anti-isomorphism $\mathcal{I}_{w}^{+}: \mathbf{R}_{q}^{+} / \mathbf{Q}_{w}^{+} \rightarrow \sum_{\lambda \in P_{+}} \mathbf{U}_{q}^{+}(w, \lambda) q^{-w \lambda}$ given by $\left[c_{f, u_{\lambda}}^{\lambda}\right] \mapsto\left(\left(\Phi^{+}\right)^{-1} \circ \bar{r}_{\geq 0}^{w}\right)\left(\left[c_{f, u_{w \lambda}}^{\lambda}\right]\right)$ for $f \in V(\lambda)^{\star}, \lambda \in P_{+}$. We have

$$
\mathcal{I}_{w}^{+}\left(\left[c_{G_{\lambda}^{\mathrm{up}}(b)^{*}, u_{\lambda}}^{\lambda}\right]\right)=G^{\mathrm{up}}\left(\bar{\jmath}_{w \lambda}^{\vee}(b)\right)^{\vee} q^{-w \lambda}=\varphi\left(D_{u_{w \lambda}, G_{\lambda}^{\mathrm{up}}(b)}\right) q^{-w \lambda}
$$

for $b \in \mathscr{B}_{w}(\lambda)$.

Corollary 5.15. The set $\mathcal{S}_{w}:=\left\{\left[c_{w \lambda, \lambda}^{\lambda}\right]\right\}_{\lambda \in P_{+}}$is an Ore set in $\mathbf{R}_{q}^{+} / \mathbf{Q}_{w}^{+}$consisting of $q$-central elements.

Remark 5.16. The description in Proposition 5.14 implies that the algebra $\mathbf{R}_{q}^{+} / \mathbf{Q}_{w}^{+}$has no zero divisors.

By Corollary [5.15, we can consider the $\mathbb{Q}(q)$-algebra $\left(\mathbf{R}_{q}^{+} / \mathbf{Q}_{w}^{+}\right)\left[\mathcal{S}_{w}^{-1}\right]$. Proposition 5.14 immediately implies the following proposition. This gives the description of $\mathbf{A}_{q}\left[N_{-}(w)\right]$ in terms of the quantized coordinate algebra $\mathbf{R}_{q}$. This description appears in Yak10, Theorem 3.7] modulo some difference of conventions.

Proposition 5.17. Let $w \in W$. Then $\mathcal{I}_{w}^{+}$induces the algebra anti-isomorphism

$$
\mathcal{I}_{w}^{+}:\left(\mathbf{R}_{q}^{+} / \mathbf{Q}_{w}^{+}\right)\left[\mathcal{S}_{w}^{-1}\right] \rightarrow \mathbf{U}_{q}^{+}(w) \check{\mathbf{U}}_{q}^{0} .
$$

Moreover the $\mathbb{Q}(q)$-algebra $\sum_{\lambda \in P_{+}}\left(\mathbf{R}_{q}^{+}(\lambda) / \mathbf{Q}_{w}^{+}\right)\left[c_{w \lambda, \lambda}^{\lambda}\right]^{-1}\left(\subset\left(\mathbf{R}_{q}^{+} / \mathbf{Q}_{w}^{+}\right)\left[\mathcal{S}_{w}^{-1}\right]\right)$ is anti-isomorphic to $\mathbf{U}_{q}^{+}(w)$, and is isomorphic to $\mathbf{A}_{q}\left[N_{-}(w)\right]$ via $\varphi$.

Proof. It suffices to show that $\sum_{\lambda \in P_{+}} \mathbf{U}_{q}^{+}(w, \lambda)=\mathbf{U}_{q}^{+}(w)$. This follows from Theorem 3.48.

5.3. Quantum twist isomorphisms and dual canonical bases. In this subsection, we prove the existence of quantum twist isomorphisms (Theorem 5.19). The following lemma easily follows from Corollary 5.11 and 5.15, See also [GW04, Proposition 6.3].

Lemma 5.18. Let $w \in W$. Then the set $\tilde{\mathcal{S}}_{w}:=\left\{q^{m}\left[c_{w \lambda, \lambda}^{\lambda} c_{\lambda^{\prime}, \lambda^{\prime}}^{\lambda^{\prime}}\right] \mid m \in \mathbb{Z}, \lambda, \lambda^{\prime} \in P_{+}\right\}$is an Ore set in $\mathbf{R}_{q}^{+} / \mathbf{Q}_{w}^{+}$consisting of $q$-central elements.

Moreover the maps $\left(\mathbf{R}_{q}^{+} / \mathbf{Q}_{w}^{+}\right)\left[\mathcal{S}^{-1}\right] \rightarrow\left(\mathbf{R}_{q}^{+} / \mathbf{Q}_{w}^{+}\right)\left[\tilde{\mathcal{S}}_{w}^{-1}\right],\left[c_{f, u_{\lambda}}^{\lambda}\right]\left[c_{\lambda^{\prime}, \lambda^{\prime}}^{\lambda^{\prime}}\right]^{-1} \mapsto\left[c_{f, u_{\lambda}}^{\lambda}\right]\left[c_{\lambda^{\prime}, \lambda^{\prime}}^{\lambda^{\prime}}\right]^{-1}$ and $\left(\mathbf{R}_{q}^{+} / \mathbf{Q}_{w}^{+}\right)\left[\mathcal{S}_{w}^{-1}\right] \rightarrow\left(\mathbf{R}_{q}^{+} / \mathbf{Q}_{w}^{+}\right)\left[\tilde{\mathcal{S}}_{w}^{-1}\right],\left[c_{f, u_{\lambda}}^{\lambda}\right]\left[c_{w \lambda^{\prime}, \lambda^{\prime}}^{\lambda^{\prime}}\right]^{-1} \mapsto\left[c_{f, u_{\lambda}}^{\lambda}\right]\left[c_{w \lambda^{\prime}, \lambda^{\prime}}^{\lambda^{\prime}}\right]^{-1}$ are injective $\mathbb{Q}(q)$ algebra homomorphisms.

Theorem 5.19. There exists an isomorphism of the $\mathbb{Q}(q)$-algebras

$$
\gamma_{w, q}: \mathbf{A}_{q}\left[N_{-}^{w}\right] \rightarrow \mathbf{A}_{q}\left[N_{-}(w) \cap w G_{0}^{\min }\right]
$$

given by

$$
\left[D_{u, u_{\lambda}}\right] \mapsto q^{-(\lambda, \mathrm{wt} u-\lambda)} D_{w \lambda, \lambda}^{-1} D_{u_{w \lambda}, u} \quad\left[D_{w \lambda, \lambda}\right]^{-1} \mapsto q^{(\lambda, w \lambda-\lambda)} D_{w \lambda, \lambda}
$$

for a weight vector $u \in V(\lambda)$ and $\lambda \in P_{+}$.

Definition 5.20. We call $\gamma_{w, q}$ a quantum twist isomorphism (cf. Proposition 2.23). 
Proof of Theorem 5.19. By Proposition 5.12 (see also Proposition [5.9), we have the algebra isomorphism

$$
\mathbf{A}_{q}\left[N_{-} \cap X_{w}\right] \stackrel{\mathcal{I}_{w}^{-1}}{\longrightarrow} \sum_{\lambda \in P_{+}}\left(\mathbf{R}_{q}^{+}(\lambda) / \mathbf{Q}_{w}^{+}\right)\left[c_{\lambda, \lambda}^{\lambda}\right]^{-1}
$$

given by

$$
\left[D_{u, u_{\lambda}}\right] \mapsto\left[c_{u^{*}, u_{\lambda}}^{\lambda}\right]\left[c_{\lambda, \lambda}^{\lambda}\right]^{-1}
$$

for $\lambda \in P_{+}$and $u \in V(\lambda)$. In particular, $\mathcal{I}_{w}^{-1}\left(\left[D_{w \lambda, \lambda}\right]\right)=\left[c_{w \lambda, \lambda}^{\lambda}\right]\left[c_{\lambda, \lambda}^{\lambda}\right]^{-1}$.

By Lemma 5.18, $\sum_{\lambda \in P_{+}}\left(\mathbf{R}_{q}^{+}(\lambda) / \mathbf{Q}_{w}^{+}\right)\left[c_{\lambda, \lambda}^{\lambda}\right]^{-1}$ is naturally regarded as a subalgebra of $\left(\mathbf{R}_{q}^{+} / \mathbf{Q}_{w}^{+}\right)\left[\tilde{\mathcal{S}}_{w}^{-1}\right]$, and in the latter algebra, the set $\left\{q^{m}\left[c_{w \lambda, \lambda}^{\lambda}\right]\left[c_{\lambda, \lambda}^{\lambda}\right]^{-1} \mid m \in \mathbb{Z}, \lambda \in P_{+}\right\}$is a multiplicative set consisting of invertible $q$-central elements. Hence the algebra isomorphism (5.1) is extended to the algebra isomorphism

$$
\mathcal{J}_{1}: \mathbf{A}_{q}\left[N_{-}^{w}\right] \rightarrow \sum_{\substack{\lambda, \lambda^{\prime}, \lambda^{\prime \prime} \in P_{+} \\ \lambda=\lambda^{\prime}+\lambda^{\prime \prime}}}\left(\mathbf{R}_{q}^{+}(\lambda) / \mathbf{Q}_{w}^{+}\right)[c_{\lambda^{\prime}, \lambda^{\prime}}^{\lambda^{\prime}} \underbrace{\lambda^{\prime \prime}}_{w \lambda^{\prime \prime}, \lambda^{\prime \prime}}]^{-1} .
$$

On the other hand, by Proposition 5.17 (see also Proposition 5.14), we have an algebra isomorphism

$$
\sum_{\lambda \in P_{+}}\left(\mathbf{R}_{q}^{+}(\lambda) / \mathbf{Q}_{w}^{+}\right)\left[c_{w \lambda, \lambda}^{\lambda}\right]^{-1} \stackrel{\varphi \circ \mathcal{I}_{w}^{+}}{\longrightarrow} \mathbf{A}_{q}\left[N_{-}(w)\right]
$$

given by

$$
\left[c_{w \lambda, \lambda}^{\lambda}\right]^{-1}\left[c_{u^{*}, u_{\lambda}}^{\lambda}\right] \mapsto D_{u_{w \lambda}, u}
$$

for $\lambda \in P_{+}$and $u \in V(\lambda)$. In particular, $\left(\varphi \circ \mathcal{I}_{w}^{+}\right)\left(\left[c_{w \lambda, \lambda}^{\lambda}\right]^{-1}\left[c_{\lambda, \lambda}^{\lambda}\right]\right)=D_{w \lambda, \lambda}$.

As above, the set $\left\{q^{m}\left[c_{w \lambda, \lambda}^{\lambda}\right]^{-1}\left[c_{\lambda, \lambda}^{\lambda}\right] \mid m \in \mathbb{Z}, \lambda \in P_{+}\right\}$is a multiplicative set consisting of invertible $q$-central elements of $\left(\mathbf{A}_{q}^{+} / \mathbf{Q}_{w}^{+}\right)\left[\tilde{\mathcal{S}}_{w}^{-1}\right]$. Hence the algebra isomorphism (5.4) is extended to the algebra isomorphism

$$
\mathcal{J}_{2}: \sum_{\substack{\lambda, \lambda^{\prime}, \lambda^{\prime \prime} \in P^{+} \\ \lambda=\lambda^{\prime}+\lambda^{\prime \prime}}}\left(\mathbf{R}_{q}^{+}(\lambda) / \mathbf{Q}_{w}^{+}\right)\left[c_{\lambda^{\prime}, \lambda^{\prime}}^{\lambda^{\prime}} c_{w \lambda^{\prime \prime}, \lambda^{\prime \prime}}^{\lambda^{\prime \prime}}\right]^{-1} \rightarrow \mathbf{A}_{q}\left[N_{-}(w) \cap w G_{0}^{\mathrm{min}}\right] .
$$

By (5.3) and (5.6), we obtain the $\mathbb{Q}(q)$-algebra isomorphism

$$
\gamma_{w, q}:=\mathcal{J}_{2} \circ \mathcal{J}_{1}: \mathbf{A}_{q}\left[N_{-}^{w}\right] \rightarrow \mathbf{A}_{q}\left[N_{-}(w) \cap w G_{0}^{\min }\right] .
$$

Moreover, for $\lambda \in P_{+}$and a weight vector $u \in V(\lambda)$, we have

$$
\begin{aligned}
\gamma_{w, q}\left(D_{u, u_{\lambda}}\right) & =\mathcal{J}_{2}\left(\left[c_{u^{*}, u_{\lambda}}^{\lambda}\right]\left[c_{\lambda, \lambda}^{\lambda}\right]^{-1}\right) \text { by }(\underline{5.2}), \\
& =\mathcal{J}_{2}\left(q^{-(\lambda, \operatorname{wt} u-\lambda)}\left[c_{\lambda, \lambda}^{\lambda}\right]^{-1}\left[c_{u^{*}, u_{\lambda}}^{\lambda}\right]\right) \text { by Proposition [5.9, } \\
& =\mathcal{J}_{2}\left(q^{-(\lambda, \operatorname{wt} u-\lambda)}\left[c_{\lambda, \lambda}^{\lambda}\right]^{-1}\left[c_{w \lambda, \lambda}^{\lambda}\right]\left[c_{w \lambda, \lambda}^{\lambda}\right]^{-1}\left[c_{u^{*}, u_{\lambda}}^{\lambda}\right]\right) \\
& =q^{-(\lambda, \operatorname{wt} u-\lambda)} D_{w \lambda, \lambda}^{-1} D_{u_{w \lambda, u}} \text { by (5.5). }
\end{aligned}
$$

Moreover,

$$
\begin{aligned}
1 & =\gamma_{w, q}\left(\left[D_{w \lambda, \lambda}\right]\left[D_{w \lambda, \lambda}\right]^{-1}\right) \\
& =q^{-(\lambda, w \lambda-\lambda)} D_{w \lambda, \lambda}^{-1} \gamma_{w, q}\left(\left[D_{w \lambda, \lambda}\right]^{-1}\right) .
\end{aligned}
$$


Hence,

$$
\gamma_{w, q}\left(\left[D_{w \lambda, \lambda}\right]^{-1}\right)=q^{(\lambda, w \lambda-\lambda)} D_{w \lambda, \lambda} .
$$

This completes the proof of the theorem.

Remark 5.21. We can also deduce Proposition 4.2 from the descriptions

$$
\left[D_{w \lambda, \lambda}\right]=\mathcal{I}_{w}\left(\left[c_{w \lambda, \lambda}^{\lambda}\right]\left[c_{\lambda, \lambda}^{\lambda}\right]^{-1}\right) \quad D_{w \lambda, \lambda}=\left(\varphi \circ \mathcal{I}_{w}^{+}\right)\left(\left[c_{w \lambda, \lambda}^{\lambda}\right]^{-1}\left[c_{\lambda, \lambda}^{\lambda}\right]\right)
$$

appearing in the proof of Theorem 5.19 together with Propositions 5.9] and 5.14,

The quantum twist isomorphism $\gamma_{w, q}$ is compatible with the dual canonical bases as follows:

Theorem 5.22. Let $w \in W$. Then the quantum twist isomorphism $\gamma_{w, q}: \mathbf{A}_{q}\left[N_{-}^{w}\right] \rightarrow \mathbf{A}_{q}\left[N_{-}(w) \cap\right.$ $\left.w G_{0}^{\text {min }}\right]$ restricts to the bijection $\widetilde{\mathbf{B}}^{\mathrm{up}, w} \rightarrow \widetilde{\mathbf{B}}^{\mathrm{up}}(w)$ given by

$$
q^{\left(\lambda, \operatorname{wt}\left(\bar{\jmath}_{\lambda^{\prime}}(b)\right)+\lambda-w \lambda\right)}\left[D_{w \lambda, \lambda}\right]^{-1}\left[G^{\text {up }}\left(\bar{\jmath}_{\lambda^{\prime}}(b)\right)\right] \mapsto q^{-\left(\lambda-\lambda^{\prime}, \operatorname{wt}\left(* \bar{\jmath}_{w \lambda^{\prime}}^{\vee}(b)\right)\right)} D_{w, \lambda-\lambda^{\prime}} G^{\text {up }}\left(* \bar{\jmath}_{w \lambda^{\prime}}^{\vee}(b)\right)
$$

for $\lambda, \lambda^{\prime} \in P_{+}, b \in \mathscr{B}_{w}\left(\lambda^{\prime}\right)$. In particular, $\gamma_{w, q}\left(\left[D_{w, \lambda}\right]\right)=D_{w,-\lambda}$ for $\lambda \in P$, and $\gamma_{w, q} \circ \sigma=$ $\sigma \circ \gamma_{w, q}$

Proof. By Proposition 3.46, for $\lambda, \lambda^{\prime} \in P_{+}$and $b \in \mathscr{B}_{w}\left(\lambda^{\prime}\right)$, we have

$$
\begin{aligned}
& \gamma_{w, q}\left(q^{\left(\lambda, \operatorname{wt}\left(\bar{\jmath}_{\lambda^{\prime}}(b)\right)+\lambda-w \lambda\right)}\left[D_{w \lambda, \lambda}\right]^{-1}\left[G^{\text {up }}\left(\bar{\jmath}_{\lambda^{\prime}}(b)\right)\right]\right) \\
& =\gamma_{w, q}\left(q^{\left(\lambda, \operatorname{wt} b-\lambda^{\prime}+\lambda-w \lambda\right)}\left[D_{w \lambda, \lambda}\right]^{-1}\left[D_{G_{\lambda^{\prime}}^{\text {up }}(b), u_{\lambda^{\prime}}}\right]\right) \\
& =q^{\left(\lambda, \operatorname{wt} b-\lambda^{\prime}+\lambda-w \lambda\right)}\left(q^{(\lambda, w \lambda-\lambda)} D_{w \lambda, \lambda}\right)\left(q^{-\left(\lambda^{\prime}, \operatorname{wt} b-\lambda^{\prime}\right)} D_{w \lambda^{\prime}, \lambda^{\prime}}^{-1} D_{u_{w \lambda^{\prime}}, G_{\lambda^{\prime}}}^{\text {up }(b)}\right) \\
& =q^{-\left(\lambda-\lambda^{\prime}, \operatorname{wt}\left(* \bar{\jmath}_{w \lambda^{\prime}}^{\vee}(b)\right)\right)} D_{w, \lambda-\lambda^{\prime}} G^{\mathrm{up}}\left(* \bar{\jmath}_{w \lambda^{\prime}}^{\vee}(b)\right) .
\end{aligned}
$$

This completes the proof.

\section{TWIST AUTOMORPhisms ON QUANTUM UNIPOTENT CELLS}

We now obtain the twist automorphisms on quantum unipotent cells.

Theorem 6.1. Let $w \in W$. Then there exists a $\mathbb{Q}(q)$-algebra automorphism

$$
\eta_{w, q}:=\iota_{w} \circ \gamma_{w, q}: \mathbf{A}_{q}\left[N_{-}^{w}\right] \rightarrow \mathbf{A}_{q}\left[N_{-}^{w}\right]
$$

given by

$$
\left[D_{u, u_{\lambda}}\right] \mapsto q^{-(\lambda, w t u-\lambda)}\left[D_{w \lambda, \lambda}\right]^{-1}\left[D_{u_{w \lambda}, u}\right] \quad\left[D_{w \lambda, \lambda}\right]^{-1} \mapsto q^{(\lambda, w \lambda-\lambda)}\left[D_{w \lambda, \lambda}\right]
$$

for a weight vector $u \in V(\lambda)$ and $\lambda \in P_{+}$. In particular, wt $\eta_{w, q}([x])=-\mathrm{wt}[x]$ for homogeneous elements $[x] \in \mathbf{A}_{q}\left[N_{-}^{w}\right]$. Moreover $\eta_{w, q}$ restricts to a permutation on the dual canonical bases $\widetilde{\mathbf{B}}^{\mathrm{up}, w}$. In particular, $\eta_{w, q}$ commutes with the dual bar involution $\sigma$, and $\eta_{w, q}\left(\left[D_{w, \lambda}\right]\right)=\left[D_{w,-\lambda}\right]$ for $\lambda \in P_{+}$.

The following follows from the theorem above and Proposition 2.23 .

Corollary 6.2. Let $w \in W$. Then the $\mathbb{Q}(q)$-algebra automorphism $\eta_{w, q}: \mathbf{A}_{q}\left[N_{-}^{w}\right] \rightarrow \mathbf{A}_{q}\left[N_{-}^{w}\right]$ induces a $\mathcal{A}$-algebra automorphism $\eta_{w, \mathcal{A}}: \mathbf{A}_{\mathbb{Q}\left[q^{ \pm 1}\right]}\left[N_{-}^{w}\right] \rightarrow \mathbf{A}_{\mathbb{Q}\left[q^{ \pm 1}\right]}\left[N_{-}^{w}\right]$ and a $\mathbb{C}$-algebra automorphism

$$
\left.\eta_{w, q}\right|_{q=1}: \mathbf{A}_{\mathbb{Q}\left[q^{ \pm 1}\right]}\left[N_{-}^{w}\right] \otimes_{\mathcal{A}} \mathbb{C} \rightarrow \mathbf{A}_{\mathbb{Q}\left[q^{ \pm 1}\right]}\left[N_{-}^{w}\right] \otimes_{\mathcal{A}} \mathbb{C} .
$$

Moreover, through the isomorphism in Corollary 4.11, the automorphism $\left.\eta_{w, q}\right|_{q=1}$ coincides with $\eta_{w}^{*}$. 
Definition 6.3. Let $w \in W$. Then we call the $\mathbb{Q}(q)$-algebra automorphism $\eta_{w, q}: \mathbf{A}_{q}\left[N_{-}^{w}\right] \rightarrow$ $\mathbf{A}_{q}\left[N_{-}^{w}\right]$ a twist automorphism on the quantum unipotent cell $N_{-}^{w}$.

Remark 6.4. In order to apply quantum twist automorphisms to a dual canonical basis element $\left[G^{\text {up }}(\tilde{b})\right], \tilde{b} \in \mathscr{B}(\infty)$, we have to find $\lambda \in P_{+}$and $b \in \mathscr{B}(\lambda)$ such that $G^{\text {up }}(\tilde{b})=D_{G_{\lambda}^{\text {up }}(b), u_{\lambda}}=$ $G^{\text {up }}\left(\bar{\jmath}_{\lambda}(b)\right)$. By Proposition 3.21, we can take $\lambda$ as $\lambda_{\tilde{b}}:=\sum_{i \in I} \varepsilon_{i}^{*}(\tilde{b}) \varpi_{i}$. Note that $\lambda_{\tilde{b}}$ is "minimal" in an appropriate sense.

\section{QuAntum TWIST AUtomorphisms AND QUANTUM CLUSTER ALGEBRAS}

In this section, we consider an additive categorification of the twist automorphism $\eta_{w, q}$ on a quantum unipotent cell $\mathbf{A}_{q}\left[N_{-}^{w}\right]$ in the sense of Geiß-Leclerc-Schröer. When $\mathfrak{g}$ is symmetric, Geiß-Leclerc-Schröer [GLS12] obtained a categorification of the twist automorphism $\eta_{w}^{*}$ on the coordinate algebra of a unipotent cell $N_{-}^{w}$ (Proposition 7.24). They used subcategories $\mathcal{C}_{w}$, introduced by Buan-Iyama-Reiten-Scott [BIRS09], of the module category of the preprojective algebra $\Pi$ corresponding to the Dynkin diagram for $\mathfrak{g}$. Geiß-Leclerc-Schröer GLS13 have also shown that the quantum unipotent subgroup $\mathbf{A}_{q}\left[N_{-}(w)\right]$ is isomorphic to a certain quantum cluster algebra $\mathscr{A}_{\mathbb{Q}(q)}\left(\mathcal{C}_{w}\right)$, which is determined by data of $\mathcal{C}_{w}$ (Proposition 7.19). Combining these results, we obtain a categorification of the twist automorphism $\eta_{w, q}$ (Theorem 7.25). See also Corollary 7.26 ,

In this section, we always consider the case that $\mathfrak{g}$ is symmetric. We assume that $\left(\alpha_{i}, \alpha_{i}\right)=2$ for all $i \in I$, and thus $q_{i}=q$ for all $i \in I$.

Notation 7.1. For $m, m^{\prime} \in \mathbb{Z}_{\geq 0}$ with $m \leq m^{\prime}$, set $\left[m, m^{\prime}\right]:=\left\{k \in \mathbb{Z} \mid m \leq k \leq m^{\prime}\right\}$.

7.1. Quantum cluster algebras. In this subsection, we briefly review quantum cluster algebras. The main references are [BZ05] and GLS13.

Definition 7.2. Let $n, \ell$ be positive integers such that $n \leq \ell$. Let $\Lambda=\left(\lambda_{i j}\right)_{i, j \in[1, \ell]}$ be a skew-symmetric integer matrix. The skew-symmetric integer matrix $\Lambda$ determines a skewsymmetric $\mathbb{Z}$-bilinear form $\mathbb{Z}^{\ell} \times \mathbb{Z}^{\ell} \rightarrow \mathbb{Z}$ by $\Lambda\left(\boldsymbol{e}_{i}, \boldsymbol{e}_{j}\right)=\lambda_{i j}$ for $i, j \in[1, \ell]$, denoted also by $\Lambda$. Here $\left\{\boldsymbol{e}_{i} \mid i \in[1, \ell]\right\}$ denotes the standard basis of $\mathbb{Z}^{\ell}$. The based quantum torus $\mathcal{T}(=\mathcal{T}(\Lambda))$ associated with $\Lambda$ is the $\mathbb{Q}\left[q^{ \pm 1 / 2}\right]$-algebra defined as follows: as a $\mathbb{Q}\left[q^{ \pm 1 / 2}\right]$-module $\mathcal{T}$ is free and has a $\mathbb{Q}\left[q^{ \pm 1 / 2}\right]$-basis $\left\{X^{\boldsymbol{a}} \mid \boldsymbol{a} \in \mathbb{Z}^{\ell}\right\}$. The multiplication is defined by

$$
X^{\boldsymbol{a}} X^{\boldsymbol{b}}=q^{\Lambda(a, b) / 2} X^{\boldsymbol{a}+\boldsymbol{b}}
$$

for $\boldsymbol{a}, \boldsymbol{b} \in \mathbb{Z}^{\ell}$. Then

- $\mathcal{T}$ is an associative algebra,

- $X^{\boldsymbol{a}} X^{\boldsymbol{b}}=q^{\Lambda(\boldsymbol{a}, \boldsymbol{b})} X^{\boldsymbol{b}} X^{\boldsymbol{a}}$ for $\boldsymbol{a}, \boldsymbol{b} \in \mathbb{Z}^{\ell}$,

- $X^{\mathbf{0}}=1$ and $\left(X^{\boldsymbol{a}}\right)^{-1}=X^{-\boldsymbol{a}}$ for $\boldsymbol{a} \in \mathbb{Z}^{\ell}$.

The based quantum torus $\mathcal{T}$ is contained in its skew-field of fractions $\mathcal{F}(=\mathcal{F}(\Lambda)$ ) [BZ05, Appendix A]. Note that $\mathcal{F}$ is a $\mathbb{Q}\left(q^{1 / 2}\right)$-algebra. Write $X_{i}:=X^{\boldsymbol{e}_{i}}$ for $i \in[1, \ell]$.

Next we define an important operation, called mutation. Let $\widetilde{B}=\left(b_{i j}\right)_{i \in[1, \ell], j \in[1, \ell-n]}$ be an $\ell \times(\ell-n)$ integer matrix. The submatrix $B=\left(b_{i j}\right)_{i, j \in[1, \ell-n]}$ of $\widetilde{B}$ is called the principal part of $\widetilde{B}$. The pair $(\Lambda, \widetilde{B})$ is said to be compatible if, for $i \in[1, \ell]$ and $j \in[1, \ell-n]$,

$$
\sum_{k=1}^{\ell} b_{k j} \lambda_{k i}=\delta_{i j} d_{j} \text { for some } d_{j} \in \mathbb{Z}_{>0} .
$$


Note that, when $(\Lambda, \widetilde{B})$ is compatible, $\widetilde{B}$ has full rank $\ell-n$ and its principal part $B=$ $\left(b_{i j}\right)_{i, j \in[1, \ell-n]}$ is skew-symmetrizable [BZ05, Proposition 3.3]. We will assume that $B$ is skewsymmetric.

For $k \in[1, \ell-n]$, define $E^{(k)}=\left(e_{i j}\right)_{i, j \in[1, \ell]}$ and $F^{(k)}=\left(f_{i j}\right)_{i, j \in[1, \ell-n]}$ as follows:

$$
e_{i j}=\left\{\begin{array}{ll}
\delta_{i, j} & \text { if } j \neq k, \\
-1 & \text { if } i=j=k, \\
\max \left(0,-b_{i k}\right) & \text { if } i \neq j=k,
\end{array} \quad f_{i j}= \begin{cases}\delta_{i, j} & \text { if } i \neq k, \\
-1 & \text { if } i=j=k, \\
\max \left(0, b_{k j}\right) & \text { if } i=k \neq j\end{cases}\right.
$$

Set

$$
\mu_{k}(\Lambda)=\left(E^{(k)}\right)^{T} \Lambda E^{(k)} \quad \mu_{k}(\widetilde{B})=E^{(k)} \widetilde{B} F^{(k)} .
$$

Then $\mu_{k}(\Lambda, \widetilde{B}):=\left(\mu_{k}(\widetilde{B}), \mu_{k}(\Lambda)\right)$ is again compatible [BZ05, Proposition 3.4]. It is said that $\mu_{k}(\Lambda, \widetilde{B})$ is obtained from $(\Lambda, \widetilde{B})$ by the mutation in direction $k$. Note that $\mu_{k}\left(\mu_{k}(\Lambda, \widetilde{B})\right)=$ $(\Lambda, \widetilde{B})$.

The pair $\mathscr{S}=\left(\left\{X_{i}\right\}_{i \in[1, \ell]}, \widetilde{B}, \Lambda\right)$ is called a quantum seed in $\mathcal{F}$, and $\left\{X_{i}\right\}_{i \in[1, \ell]}$ is called the quantum cluster of $\mathscr{S}$. For $k \in[1, \ell-n]$, define $\mu_{k}\left(\left\{X_{i}\right\}_{i \in[1, \ell]}\right)=\left\{X_{i}^{\prime}\right\}_{i \in[1, \ell]} \subset \mathcal{F} \backslash\{0\}$ by

- $X_{i}^{\prime}=X_{i}$ if $i \neq k$,

- $X_{k}^{\prime}=X^{-\boldsymbol{e}_{k}+\sum_{j ; b_{j k}>0} b_{j k} \boldsymbol{e}_{j}}+X^{-\boldsymbol{e}_{k}-\sum_{j ;-b_{j k}>0} b_{j k} \boldsymbol{e}_{j}}$.

Then there is an injective $\mathbb{Q}\left[q^{ \pm 1 / 2}\right]$-algebra homomorphisms $\mathcal{T}\left(\mu_{k}(\Lambda)\right) \rightarrow \mathcal{F}(\Lambda)$ given by $X_{i}^{ \pm 1} \mapsto\left(X_{i}^{\prime}\right)^{ \pm 1}(i \in[1, \ell])$. Moreover there exist a basis $\left\{\boldsymbol{c}_{i}\right\}_{i \in[1, \ell]}$ of $\mathbb{Z}^{\ell}$ and a $\mathbb{Q}\left(q^{1 / 2}\right)$-algebra automorphism $\tau: \mathcal{F}(\Lambda) \rightarrow \mathcal{F}(\Lambda)$ such that $\tau\left(X^{c_{i}}\right)=X_{i}^{\prime}$ for $i \in[1, \ell$ ] BZ05, Proposition 4.7]. Hence the map above is extended to the isomorphism $\mathcal{F}\left(\mu_{k}(\Lambda)\right) \rightarrow \mathcal{F}(\Lambda)$. Through this isomorphism, we identify $\mathcal{F}\left(\mu_{k}(\Lambda)\right)$ with $\mathcal{F}(\Lambda)$, and henceforth always write $\mathcal{F}$ for this skew-field. Write

$$
\mu_{k}(\mathscr{S}):=\left(\mu_{k}\left(\left\{X_{i}\right\}_{i \in[1, \ell]}\right), \mu_{k}(\widetilde{B}), \mu_{k}(\Lambda)\right)
$$

and this is called a quantum seed obtained from the mutation of $\mathscr{S}$ in direction $k$. Note that $\mu_{k}\left(\mu_{k}\left(\mathscr{S}^{\prime}\right)\right)=\mathscr{S}^{\prime}$ for any quantum seed $\mathscr{S}^{\prime}$ and $k \in[1, \ell-n]$. By the argument above, we can consider the iterated mutations in arbitrary various directions $k \in[1, \ell-n]$. The subset $\left\{X_{i} \mid i \in[\ell-n+1, \ell]\right\}$, called the set of frozen variables, is contained in the quantum cluster of an arbitrary seed obtained by iterated mutations of $\mathscr{S}$.

The quantum cluster algebra $\mathscr{A}_{q^{ \pm 1 / 2}}(\mathscr{S})$ is defined as the $\mathbb{Q}\left[q^{ \pm 1 / 2}\right]$-subalgebra of $\mathcal{F}$ generated by the union of the quantum clusters of all quantum seeds obtained by iterated mutations of $\mathscr{S}$. An element $M \in \mathscr{A}_{q^{ \pm 1 / 2}}(\mathscr{S})$ is called a quantum cluster monomial if there exists a quantum cluster $\left\{X_{i}^{\prime}=\left(X^{\prime}\right)^{\boldsymbol{e}_{i}}\right\}_{i \in[1, \ell]}$ of a quantum seed obtained by iterated mutations of $\mathscr{S}$ such that $M=\left(X^{\prime}\right)^{\boldsymbol{a}}$ for some $\boldsymbol{a} \in \mathbb{Z}_{\geq 0}^{\ell}$.

Proposition 7.3 ([BZ05, Corollary 5.2]). The quantum cluster algebra $\mathscr{A}_{q^{ \pm 1 / 2}}(\mathscr{S})$ is contained in the based quantum torus generated by the quantum cluster of an arbitrary quantum seed obtained by iterated mutations of $\mathscr{S}$.

7.2. Quantum cluster algebra structures on quantum unipotent subgroups and quantum unipotent cells. In this subsection, we review the construction of the quantum cluster algebra structure on $\mathbf{A}_{q}\left[N_{-}(w)\right]$ following [GLS11, GLS12, GLS13]. We note that our convention is slightly different from Geiß-Leclerc-Schröer's one, see Remark 7.16. 
Definition 7.4. A finite quiver $\mathbf{Q}=\left(\mathbf{Q}_{0}, \mathbf{Q}_{1}, s, t\right)$ is a datum such that

(1) $\mathbf{Q}_{0}$ is a finite set, called the set of vertices,

(2) $\mathbf{Q}_{1}$ is a finite set, called the set of arrows,

(3) $s, t: \mathbf{Q}_{1} \rightarrow \mathbf{Q}_{0}$ are maps, and it is said that $a \in \mathbf{Q}_{1}$ is an arrow from $s(a)$ to $t(a)$.

Here we take a finite quiver $\mathbf{Q}$ such that $\mathbf{Q}_{0}=I, s(a) \neq t(a)$ for all $a \in \mathbf{Q}_{1}$ and $a_{i j}(:=$ $\left.\left\langle h_{i}, \alpha_{j}\right\rangle\right)=-\#\left\{a \in \mathbf{Q}_{1} \mid s(a)=i, t(a)=j\right\}-\#\left\{a \in \mathbf{Q}_{1} \mid s(a)=j, t(a)=i\right\}$. Such a quiver $\mathbf{Q}$ is called a finite quiver without edge loops which corresponds to the symmetric generalized Cartan matrix $A$ [GLS11, Subsection 2.1 and 4.1]. Let $\overline{\mathbf{Q}}=\left(\mathbf{Q}_{0}, \overline{\mathbf{Q}}_{1}:=\mathbf{Q}_{1} \coprod \mathbf{Q}_{1}^{*}, s, t\right)$ be the double quiver of $\mathbf{Q}$, which is obtained from $\mathbf{Q}$ by adding to each arrow $a \in \mathbf{Q}_{1}$ an arrow $a^{*} \in \mathbf{Q}_{1}^{*}$ such that $s\left(a^{*}\right)=t(a)$ and $t\left(a^{*}\right)=s(a)$. Set

$$
\Pi:=\mathbb{C} \overline{\mathbf{Q}} /\left(\sum_{a \in \mathbf{Q}_{1}}\left(a^{*} a-a a^{*}\right)\right),
$$

Here $\mathbb{C} \overline{\mathbf{Q}}$ is a path algebra of $\overline{\mathbf{Q}}$, and $\left(\sum_{a \in \mathbf{Q}_{1}}\left(a^{*} a-a a^{*}\right)\right)$ is the two-sided ideal generated by $\sum_{a \in \mathbf{Q}_{1}}\left(a^{*} a-a a^{*}\right)$. This is called the preprojective algebra associated with $\mathbf{Q}$. Denote by $\epsilon_{i}$ the idempotent of $\Pi$ corresponding to $i \in I$. For a finite dimensional $\Pi$-module $X$, write $\underline{\operatorname{dim}} X:=-\sum_{i \in I}\left(\operatorname{dim}_{\mathbb{C}} \epsilon_{i} . X\right) \alpha_{i} \in Q_{-}$. Remark that we do not regard $\underline{\operatorname{dim}} X$ as an element of $Q_{+}$. A finite dimensional $\Pi$-module $X$ is said to be nilpotent if there exists $N \in \mathbb{Z}_{\geq 0}$ such that $a_{1} \cdots a_{N} \cdot X=0$ for any sequence $\left(a_{1}, \ldots, a_{N}\right) \in \overline{\mathbf{Q}}_{1}^{N}$.

Proposition 7.5 ([CB00, Lemma 1]). For any finite dimensional $\Pi$-module $X, Y$, we have

$$
(\underline{\operatorname{dim}} X, \underline{\operatorname{dim}} Y)=\operatorname{dim}_{\mathbb{C}} \operatorname{Hom}_{\Pi}(X, Y)+\operatorname{dim}_{\mathbb{C}} \operatorname{Hom}_{\Pi}(Y, X)-\operatorname{dim}_{\mathbb{C}} \operatorname{Ext}_{\Pi}^{1}(X, Y) .
$$

Remark 7.6. For any finite dimensional П-modules $X, Y$, we have

$$
\operatorname{dim}_{\mathbb{C}} \operatorname{Ext}_{\Pi}^{1}(X, Y)=\operatorname{dim}_{\mathbb{C}} \operatorname{Ext}_{\Pi}^{1}(Y, X)
$$

by Proposition 7.5 ,

A finite dimensional nilpotent $\Pi$-module $X$ determines an element $\varphi_{X}$ of $\mathbb{C}\left[\boldsymbol{N}_{-}\right]=\mathbf{U}\left(\mathfrak{n}_{-}\right)_{\text {gr }}^{*}$ through Lusztig's construction of the universal enveloping algebra $\mathbf{U}\left(\mathfrak{n}_{-}\right)$as a space $\mathcal{M}$ consisting of certain constructible functions with convolution product [Lus00. There is a short summary, for instance, in GLS11, Subsection 2.2]. However we remark that, in this paper, we consider the convolution product on $\mathcal{M}$ opposite to the one in GLS11, Subsection 2.2]. See also Remark 7.16. The following are important properties of $\varphi_{X}$.

Proposition 7.7 ([Lus00, GLS05, GLS07b]). Let X, Y be finite dimensional nilpotent Пmodules. The following hold:

(1) wt $\varphi_{X}=\underline{\operatorname{dim}} X$.

(2) $\varphi_{X} \varphi_{Y}=\varphi_{X \oplus Y}$.

(3) Suppose that $\operatorname{dim}_{\mathbb{C}} \operatorname{Ext}_{\Pi}^{1}(X, Y)=1$. Write non-split short exact sequences as

$$
0 \rightarrow X \rightarrow Z_{1} \rightarrow Y \rightarrow 0 \quad 0 \rightarrow Y \rightarrow Z_{2} \rightarrow X \rightarrow 0 .
$$

Then we have $\varphi_{X} \varphi_{Y}=\varphi_{Z_{1}}+\varphi_{Z_{2}}$. 
Notation 7.8. Let $w \in W$ and fix $\boldsymbol{i}=\left(i_{1}, \ldots, i_{\ell}\right) \in I(w)$. Then, for $k=1, \ldots, \ell$, we set

$$
\begin{aligned}
k^{+} & :=\min \left(\{\ell+1\} \cup\left\{k+1 \leq j \leq \ell \mid i_{j}=i_{k}\right\}\right), \\
k^{-} & :=\max \left(\{0\} \cup\left\{1 \leq j \leq k-1 \mid i_{j}=i_{k}\right\}\right), \\
k^{\max } & :=\max \left\{1 \leq j \leq \ell \mid i_{j}=i_{k}\right\}, \\
k^{\mathrm{min}} & :=\min \left\{1 \leq j \leq \ell \mid i_{j}=i_{k}\right\} .
\end{aligned}
$$

Moreover, set $I_{w}:=\left\{i \in I \mid i=i_{k}\right.$ for some $\left.k=1, \ldots, \ell\right\}$. Then we can easily check that $I_{w}$ does not depend on the choice of $\boldsymbol{i}$.

Definition 7.9. Let $S_{i}$ be the (simple) $\Pi$-module such that $\underline{\operatorname{dim}} S_{i}=-\alpha_{i}$ for $i \in I$. For a $\Pi$-module $X$ and $i \in I$, define $\operatorname{soc}_{i}(X) \subset X$ by the sum of all submodules of $X$ isomorphic to $S_{i}$. For a sequence $\left(i_{1}, \ldots, i_{k}\right) \in I^{k}\left(k \in \mathbb{Z}_{>0}\right)$, there exists a unique chain

$$
X \supset X_{0} \supset X_{1} \supset X_{2} \supset \cdots \supset X_{k}=0
$$

of submodules of $X$ such that $X_{j-1} / X_{j} \simeq \operatorname{soc}_{i_{j}}\left(X / X_{j}\right)$ for $j=1, \ldots, k$. Set $\operatorname{soc}_{\left(i_{1}, \ldots, i_{k}\right)}(X):=$ $X_{0}$. For $i \in I$, denote by $\hat{I}_{i}$ the indecomposable injective $\Pi$-module with socle $S_{i}$. Let $w \in W$ and $\boldsymbol{i}=\left(i_{1}, \ldots, i_{\ell}\right) \in I(w)$. For $k=1, \ldots, \ell$, set

$$
V_{\boldsymbol{i}, k}:=\operatorname{soc}_{\left(i_{1}, \ldots, i_{k}\right)}\left(\hat{I}_{i_{k}}\right)
$$

Set $V_{\boldsymbol{i}}:=\bigoplus_{k=1, \ldots, \ell} V_{i, k}$. Define $\mathcal{C}_{w}$ as a full subcategory of the category of $\Pi$-modules consisting of all $\Pi$-modules $X$ such that there exist $t \in \mathbb{Z}_{>0}$ and a surjective homomorphism $V_{\boldsymbol{i}}^{\oplus t} \rightarrow X$. Then it is known that $\mathcal{C}_{w}$ does not depend on the choice of $\boldsymbol{i} \in I(w)$. Note that all objects of $\mathcal{C}_{w}$ are nilpotent $\Pi$-modules. An object $C \in \mathcal{C}_{w}$ is called $\mathcal{C}_{w}$-projective (resp. $\mathcal{C}_{w^{-}}$ injective) if $\operatorname{Ext}_{\Pi}^{1}(C, X)=0$ (resp. $\operatorname{Ext}_{\Pi}^{1}(X, C)=0$ ) for all $X \in \mathcal{C}_{w}$. The category $\mathcal{C}_{w}$ is closed under extension and is Frobenius. In particular, an object $X \in \mathcal{C}_{w}$ is $\mathcal{C}_{w}$-projective if and only if it is $\mathcal{C}_{w}$-injective. An object $T$ of $\mathcal{C}_{w}$ is called $\mathcal{C}_{w}$-maximal rigid if $\operatorname{Ext}_{\Pi}^{1}(T \oplus X, X)=0$ with $X \in \mathcal{C}_{w}$ implies that $X$ is isomorphic to a direct summand of a direct sum of copies of $T$. A $\Pi$-module $M$ is called basic if it is written as a direct sum of pairwise non-isomorphic indecomposable modules. Then, in fact, $V_{\boldsymbol{i}}$ is a basic $\mathcal{C}_{w}$-maximal rigid module and $V_{\boldsymbol{i}, k^{\max }}$ is the $\mathcal{C}_{w}$-projective-injective module with socle $S_{i_{k}}$ for $k=1, \ldots, \ell$. See [BIRS09] for more details, and [GLS11, Subsection 2.4] for more detailed summaries.

Let $T$ be a basic $\mathcal{C}_{w}$-maximal rigid module and $T=T_{1} \oplus \cdots \oplus T_{\ell}$ its indecomposable decomposition. From now on, we write $I_{w}=[1, n]$ for simplicity, and always number indecomposable summands of $T$ so that $T_{\ell-n+i}, i \in I_{w}$ is the $\mathcal{C}_{w}$-projective-injective module with socle $S_{i}$. Note that this labelling is different from the labelling $V_{\boldsymbol{i}}=\bigoplus_{k \in[1, \ell]} V_{\boldsymbol{i}, k}$. Let $\Gamma_{T}$ be the Gabriel quiver of $A_{T}:=\operatorname{End}_{\Pi}(T)^{\mathrm{op}}$, that is, the vertex set of $\Gamma_{T}$ is $[1, \ell]$ and $d_{i j}:=\operatorname{dim}_{\mathbb{C}} \operatorname{Ext}_{A_{T}}^{1}\left(S_{T_{i}}, S_{T_{j}}\right)$ arrows from $i$ to $j$, where $S_{T_{i}}$ is the head of a (projective) $A_{T^{-}}$ module $\operatorname{Hom}_{\Pi}\left(T, T_{i}\right)$. Define $\widetilde{B}_{T}=\left(b_{i j}\right)_{i \in[1, \ell], j \in[1, \ell-n]}$ by $b_{i j}:=d_{j i}-d_{i j}$. The following proposition is an essential results for the additive categorification of cluster algebras.

Proposition 7.10 ([BIRS09, GLS07a]). In the setting above, the following hold:

(1) $\ell=\ell(w)$.

(2) For any $k \in[1, \ell-n]$, there exists a unique indecomposable $\Pi$-module in $\mathcal{C}_{w}$ such that $T_{k}^{*} \not T_{k}$ and $\left(T / T_{k}\right) \oplus T_{k}^{*}$ is a basic $\mathcal{C}_{w}$-maximal rigid module. This basic $\mathcal{C}_{w}$-maximal rigid module is denoted by $\mu_{T_{k}}(T)$ and called the mutation of $T$ in direction $T_{k}$.

(3) For any $k \in[1, \ell-n], \mu_{k}\left(\widetilde{B}_{T}\right)=\widetilde{B}_{\mu_{T_{k}}(T)}$. 
(4) For any $k \in[1, \ell-n]$, we have $\operatorname{dim}_{\mathbb{C}} \operatorname{Ext}_{\Pi}^{1}\left(T_{k}, T_{k}^{*}\right)=1$, and there exists non-split exact sequences

$$
0 \rightarrow T_{k} \rightarrow T_{-} \rightarrow T_{k}^{*} \rightarrow 0 \quad 0 \rightarrow T_{k}^{*} \rightarrow T_{+} \rightarrow T_{k} \rightarrow 0
$$

such that $T_{-} \simeq \bigoplus_{j ; b_{j k}<0} T_{j}^{\oplus\left(-b_{j k}\right)}$ and $T_{+} \simeq \bigoplus_{j ; b_{j k}>0} T_{j}^{\oplus b_{j k}}$.

Note that, by Proposition 7.7 and 7.10, we have

$$
\varphi_{T_{k}} \varphi_{T_{k}^{*}}=\varphi_{T_{+}}+\varphi_{T_{-}}=\prod_{j ; b_{j k}>0} \varphi_{T_{j}}^{b_{j k}}+\prod_{j ; b_{j k}<0} \varphi_{T_{j}}^{-b_{j k}} .
$$

This is an additive categorification of mutation. See [GLS11, Subsection 2.7] and references therein for more details. An object $T$ of $\mathcal{C}_{w}$ is said to be reachable (in $\mathcal{C}_{w}$ ) if $T$ is isomorphic to a direct summand of a direct sum of copies of a basic $\mathcal{C}_{w}$-maximal rigid module which is obtained from $V_{\boldsymbol{i}}$ by iterated mutations. In fact, the notion of reachable does not depend on the choice of $\boldsymbol{i}$ [BIRS09, Proposition III.4.3].

Remark 7.11. Let $T$ be a basic reachable $\mathcal{C}_{w}$-maximal rigid module, and $T=T_{1} \oplus \cdots \oplus T_{\ell}$ its indecomposable decomposition. By Proposition [7.5, for any $i, j \in[1, \ell]$, we have

$$
\left(\underline{\operatorname{dim}} T_{i}, \underline{\operatorname{dim}} T_{j}\right)=\operatorname{dim}_{\mathbb{C}} \operatorname{Hom}_{\Pi}\left(T_{i}, T_{j}\right)+\operatorname{dim}_{\mathbb{C}} \operatorname{Hom}_{\Pi}\left(T_{j}, T_{i}\right) .
$$

Definition 7.12. Let $\boldsymbol{i}=\left(i_{1}, \ldots, i_{\ell}\right) \in I(w)$. For $1 \leq a \leq b \leq \ell$ with $i_{a}=i_{b}$, there exists a natural injective homomorphism $V_{\boldsymbol{i}, a^{-}} \rightarrow V_{\boldsymbol{i}, b}$ of $\Pi$-modules, and the cokernel of this homomorphism is denoted by $M_{\boldsymbol{i}}[b, a]$. Here we set $V_{\boldsymbol{i}, 0}:=0$. In particular, $M_{\boldsymbol{i}}\left[b, b^{\mathrm{min}}\right]$ is isomorphic to $V_{\boldsymbol{i}, b}$. Geiß-Leclerc-Schröer shows that $M_{\boldsymbol{i}}[b, a]$ is reachable for all $1 \leq a \leq b \leq \ell$ with $i_{a}=i_{b}$ GLS11, section 13].

We use the notation in Definition 7.9. Geiß-Leclerc-Schröer construct a quantum cluster algebra $\mathscr{A}_{\mathbb{Q}(q)}\left(\mathcal{C}_{w}\right)$ associated with $\mathcal{C}_{w}$ as we shall recall. Let $T$ be a basic $\mathcal{C}_{w}$-maximal rigid module and $T=T_{1} \oplus \cdots \oplus T_{\ell}$ its indecomposable decomposition. Define $\Lambda_{T}:=\left(\lambda_{i j}\right)_{i, j \in[1, \ell]}$ by

$$
\lambda_{i j}:=\operatorname{dim}_{\mathbb{C}} \operatorname{Hom}_{\Pi}\left(T_{i}, T_{j}\right)-\operatorname{dim}_{\mathbb{C}} \operatorname{Hom}_{\Pi}\left(T_{j}, T_{i}\right) .
$$

Geiß-Leclerc-Schröer have shown the following properties:

Proposition 7.13 (GLS13, Proposition 10.1, Proposition 10.2]). (1) $\left(\widetilde{B}_{T}, \Lambda_{T}\right)$ is compatible in the sense of Definition 7.2.

$(2) \mu_{k}\left(\widetilde{B}_{T}, \Lambda_{T}\right)=\left(\widetilde{B}_{\mu_{T_{k}}(T)}, \Lambda_{\mu_{T_{k}}(T)}\right)$ for $k \in[1, \ell-n]$.

Definition 7.14. The quantum cluster algebra $\mathscr{A}_{q^{ \pm 1 / 2}}\left(\mathcal{C}_{w}\right)$ is defined as the quantum cluster algebra with the initial seed $\left.\left(\left(X_{T}\right)_{i}\right)_{i \in[1, \ell]}, \widetilde{B}_{T}, \Lambda_{T}\right)$ for a basic reachable $\mathcal{C}_{w}$-maximal rigid module $T$. Note that this algebra $\mathscr{A}_{q^{ \pm 1 / 2}}\left(C_{w}\right)$ does not depend on the choice of $T$. By the properties above, we may write

$$
\mu_{k}\left(\left(\left(X_{T}\right)_{i}\right)_{i \in[1, \ell]}, \widetilde{B}_{T}, \Lambda_{T}\right)=\left(\left(\left(X_{\mu_{T_{k}}(T)}\right)_{i}\right)_{i \in[1, \ell]}, \widetilde{B}_{\mu_{T_{k}}(T)}, \Lambda_{\mu_{T_{k}}(T)}\right)
$$

for $k \in[1, \ell-n]$. Moreover, for $\boldsymbol{a}=\left(a_{1}, \ldots, a_{\ell}\right) \in \mathbb{Z}_{\geq 0}^{\ell}$, set $X_{\bigoplus_{i \in[1, \ell]} T_{i}^{\oplus a_{i}}}:=\left(X_{T}\right)^{\boldsymbol{a}}$. Then the quantum cluster monomials of $\mathscr{A}_{q^{ \pm 1 / 2}}\left(\mathcal{C}_{w}\right)$ are indexed by reachable $\Pi$-modules in $\mathcal{C}_{w}$.

Set

$$
Y_{R}:=q \underline{(\underline{\operatorname{dim}} R, \underline{\operatorname{dim}} R) / 4} X_{R}
$$


for every reachable $\Pi$-module $R$ in $\mathcal{C}_{w}$. Recall that $\underline{\operatorname{dim}} R \in Q_{-}$. Define the rescaled quantum cluster algebra $\mathscr{A}_{q^{ \pm 1}}\left(\mathcal{C}_{w}\right)$ as an $\mathcal{A}\left(:=\mathbb{Q}\left[q^{ \pm 1}\right]\right)$-subalgebra of $\mathscr{A}_{q^{ \pm 1 / 2}}\left(\mathcal{C}_{w}\right)$ generated by $\left\{Y_{R} \mid\right.$ $R$ is reachable in $\left.\mathcal{C}_{w}\right\}$. For any basic reachable $\mathcal{C}_{w}$-maximal rigid module $T=T_{1} \oplus \cdots \oplus T_{\ell}$, the rescaled quantum cluster algebra $\mathscr{A}_{q^{ \pm 1}}\left(\mathcal{C}_{w}\right)$ is contained in the rescaled based quantum torus $\mathcal{T}_{\mathcal{A}, T}:=\mathcal{A}\left[Y_{T_{k}}^{ \pm 1} \mid k \in[1, \ell]\right](\subset \mathcal{F})$ GLS13, Lemma 10.4 and Proposition 10.5] (they are cited as (7.2) and Proposition 7.17 below). Note that, for $\left(a_{1}, \ldots, a_{\ell}\right) \in \mathbb{Z}_{\geq 0}^{\ell}$, we have

$$
Y_{R}=q^{\alpha(R)} Y_{T_{1}}^{a_{1}} \cdots Y_{T_{\ell}}^{a_{\ell}}
$$

here we set $R:=\bigoplus_{i \in[1, \ell]} T_{i}^{\oplus a_{i}}$ and

$$
\alpha(R):=\sum_{i \in[1, \ell]} a_{i}\left(a_{i}-1\right) \operatorname{dim}_{\mathbb{C}} \operatorname{Hom}_{\Pi}\left(T_{i}, T_{i}\right) / 2+\sum_{i<j ; i, j \in[1, \ell]} a_{i} a_{j} \operatorname{dim}_{\mathbb{C}} \operatorname{Hom}_{\Pi}\left(T_{j}, T_{i}\right) .
$$

Note that $\mathbf{I}:=\left\{q^{m} Y_{\bigoplus_{i \in[\ell-n+1, \ell]} T_{i}^{a_{i}}} \mid\left(a_{\ell-n+1}, \ldots, a_{\ell}\right) \in \mathbb{Z}_{\geq 0}^{n}, m \in \mathbb{Z}\right\}$ is an Ore set in $\mathscr{A}_{q^{ \pm 1}}\left(\mathcal{C}_{w}\right)$. Set $\widetilde{\mathscr{A}}_{q^{ \pm 1}}\left(\mathcal{C}_{w}\right):=\mathscr{A}_{q^{ \pm 1}}\left(\mathcal{C}_{w}\right)\left[\mathbf{I}^{-1}\right]$, and $\mathscr{A}_{\mathbb{Q}(q)}\left(\mathcal{C}_{w}\right):=\mathbb{Q}(q) \otimes_{\mathcal{A}} \mathscr{A}_{q^{ \pm 1}}\left(\mathcal{C}_{w}\right), \widetilde{\mathscr{A}}_{\mathbb{Q}(q)}\left(\mathcal{C}_{w}\right):=\mathbb{Q}(q) \otimes_{\mathcal{A}}$ $\widetilde{\mathscr{A}_{q} \pm 1}\left(\mathcal{C}_{w}\right)$.

For $X \in \mathcal{C}_{w}$, denote by $I(X)$ the injective hull of $X$ in $\mathcal{C}_{w}$, and by $\Omega_{w}^{-1}(X)$ the cokernel of the corresponding injective homomorphism $X \rightarrow I(X)$. Hence we have an exact sequence

$$
0 \rightarrow X \rightarrow I(X) \rightarrow \Omega_{w}^{-1}(X) \rightarrow 0 .
$$

Proposition 7.15 ([GLS11, Proposition 13.4]). Let $w \in W, T$ a basic reachable $\mathcal{C}_{w}$-maximal rigid module and $T=T_{1} \oplus \cdots \oplus T_{\ell}$ its indecomposable decomposition. Then $T^{\prime}:=\Omega_{w}^{-1}(T) \oplus$ $\bigoplus_{i \in I_{w}} T_{\ell-n+i}$ is also a basic reachable $\mathcal{C}_{w}$-maximal rigid module; hence there exists a bijection $[1, \ell-n] \rightarrow[1, \ell-n], k \mapsto k^{*}$ such that $T_{k^{*}}^{\prime}=\Omega_{w}^{-1}\left(T_{k}\right)$.

Let $k \in[1, \ell-n]$ and write $\mu_{T_{k}}(T)=\left(T / T_{k}\right) \oplus T_{k}^{*}$. Then we have

$$
\mu_{T_{k^{*}}^{\prime}}\left(T^{\prime}\right)=\left(T^{\prime} / T_{k^{*}}^{\prime}\right) \oplus \Omega_{w}^{-1}\left(T_{k}^{*}\right) .
$$

Remark 7.16. Let $w \in W$. In this remark, we explain the difference between our convention and Geiß-Leclerc-Schröer's one in GLS11, GLS12, GLS13]. An object $\mathcal{X}$ in Geiß-LeclercSchröer's papers is denoted by $\mathcal{X}^{\text {GLS }}$ here.

The category $\mathcal{C}_{w}$ is the same category as $\mathcal{C}_{w^{-1}}^{\mathrm{GLS}}$. Moreover $N_{-}(w)=\left(N\left(w^{-1}\right)^{\mathrm{GLS}}\right)^{T}$ and $N_{-}^{w}=\left(\left(N^{w^{-1}}\right)^{\mathrm{GLS}}\right)^{T}$. We omitted the definition of $\varphi_{X}$ for a finite dimensional nilpotent $\Pi$-module $X$, however the algebra $\mathcal{M}$ used for its precise definition (see Definition [7.4) is the same space as $\mathcal{M}^{\mathrm{GLS}}$ in [GLS11, Subsection 2.2] equipped with the opposite convolution product.

Thus there exist algebra isomorphisms $\mathbb{C}\left[N_{-}(w)\right] \rightarrow \mathbb{C}\left[N\left(w^{-1}\right)^{\mathrm{GLS}}\right]$ and $\mathbb{C}\left[N_{-}^{w}\right] \rightarrow \mathbb{C}\left[N^{w^{-1}, \mathrm{GLS}}\right]$ given by $f \rightarrow f \circ(-)^{T}$. Moreover $\varphi_{X}=\varphi_{X}^{\mathrm{GLS}} \circ(-)^{T}$ for all $X \in \mathcal{C}_{w}=\mathcal{C}_{w^{-1}}^{\mathrm{GLS}}$. See also GLS11, Chapter 6]. (This is the reason why we consider the opposite product on $\mathcal{M}$.)

The quantum nilpotent subalgebra $\mathbf{U}_{q}\left(\mathfrak{n}\left(w^{-1}\right)\right)^{\mathrm{GLS}}$ in [GLS13 is equal to $\mathbf{A}_{q}\left[N_{-}(w)\right]^{\vee}$. Geiß-Leclerc-Schröer consider a $\mathbb{Q}(q)$-algebra $\mathbf{A}_{q}\left(\mathfrak{n}\left(w^{-1}\right)\right)^{\text {GLS }}$, called the quantum coordinate ring, which is defined in $\left(\mathbf{U}_{q}^{+}\right)^{*}$ GLS13, (4.6)], and define an algebra isomorphism $\Psi^{\mathrm{GLS}}: \mathbf{U}_{q}\left(\mathfrak{n}\left(w^{-1}\right)\right)^{\mathrm{GLS}} \rightarrow \mathbf{A}_{q}\left(\mathfrak{n}\left(w^{-1}\right)\right)^{\mathrm{GLS}}$ by using a non-degenerate bilinear form $(,)^{\text {GLS }}$ 
GLS13, Proposition 4.1]. Actually, for $x \in\left(\mathbf{U}_{q}^{+}\right)_{\beta}, y \in\left(\mathbf{U}_{q}^{+}\right)_{\beta^{\prime}}\left(\beta, \beta^{\prime} \in Q_{+}\right)$, we have

$$
\begin{aligned}
(x, y)^{\mathrm{GLS}} & =\delta_{\beta, \beta^{\prime}}\left(1-q^{-2}\right)^{\mathrm{ht} \beta} \overline{(\bar{x}, \bar{y})_{L}^{+}} \\
& =\delta_{\beta, \beta^{\prime}}\left(1-q^{-2}\right)^{\mathrm{ht} \beta} \overline{\left(\overline{x^{\vee}}, \overline{y^{\vee}}\right)_{L}} \\
& =\delta_{\beta, \beta^{\prime}}\left(1-q^{-2}\right)^{\mathrm{ht} \beta}\left(x^{\vee}, \sigma\left(\overline{y^{\vee}}\right)\right)_{L} \\
& =q^{(\beta, \beta) / 2}\left(q^{-1}-q\right)^{\mathrm{ht} \beta}\left(x^{\vee}, \varphi(y)\right)_{L} .
\end{aligned}
$$

The last equality follows from Proposition [3.10. By the way, there exists a $\mathbb{Q}(q)$-algebra automorphism $m_{\text {norm }}: \mathbf{U}_{q}^{-} \rightarrow \mathbf{U}_{q}^{-}$given by $f_{i} \mapsto\left(q^{-1}-q\right)^{-1} f_{i}$ for $i \in I$. We now have the following $\mathbb{Q}(q)$-algebra isomorphism;

$$
I_{\text {norm }}: \mathbf{A}_{q}\left[N_{-}(w)\right] \stackrel{m_{\mathrm{norm}}}{\longrightarrow} \mathbf{A}_{q}\left[N_{-}(w)\right] \stackrel{\vee}{\rightarrow} \mathbf{U}_{q}\left(\mathfrak{n}\left(w^{-1}\right)\right)^{\mathrm{GLS}} \stackrel{\Psi^{\mathrm{GLS}}}{\longrightarrow} \mathbf{A}_{q}\left(\mathfrak{n}\left(w^{-1}\right)\right)^{\mathrm{GLS}},
$$

which maps $x \in\left(\mathbf{U}_{q}^{-}\right)_{\beta}\left(\beta \in-Q_{+}\right)$to $q^{(\beta, \beta) / 2}(x, \varphi(-))_{L}$. By using this isomorphism, we describe their results. Note that $I_{\text {norm }}\left(D_{w \lambda, w^{\prime} \lambda}\right)=q^{\left(w \lambda-w^{\prime} \lambda, w \lambda-w^{\prime} \lambda\right) / 2} D_{w^{\prime} \lambda, w \lambda}^{\mathrm{GLS}}$ for $w, w^{\prime} \in W$ and $\lambda \in P_{+}$[GLS13, (5.5)].

The definitions of the quantum cluster algebra $\mathscr{A}_{q^{ \pm 1 / 2}}\left(\mathcal{C}_{w}\right)=\mathscr{A}_{q^{ \pm 1 / 2}}\left(\mathcal{C}_{w^{-1}}^{\mathrm{GLS}}\right)$ are the same. We have $Y_{R}=q \underline{(\underline{\operatorname{dim}} R, \underline{\operatorname{dim}} R) / 2} Y_{R}^{\mathrm{GLS}}$ for every reachable $\Pi$-module $R$ [GLS13, (10.16)]. Note that $(\underline{\operatorname{dim}} R, \underline{\operatorname{dim}} R) / 2 \in \mathbb{Z}$. Therefore we have $\mathscr{A}_{q^{ \pm 1}}\left(\mathcal{C}_{w}\right)=\mathscr{A}_{\mathbb{A}}\left(\mathcal{C}_{w^{-1}}^{\mathrm{GLS}}\right)^{\mathrm{GLS}}$.

The following propositions describe mutations of quantum clusters and twisted dual bar involutions in $\mathscr{A}_{q^{ \pm 1}}\left(\mathcal{C}_{w}\right)$.

Proposition 7.17 (GLS13, Proposition 10.5]). Let $T$ be a basic reachable $\mathcal{C}_{w}$-maximal rigid module, and $T=T_{1} \oplus \cdots \oplus T_{\ell}$ its indecomposable decomposition. Fix $k \in[1, \ell-n]$. Write $\tilde{B}_{T}=\left(b_{i j}\right)_{i \in[1, \ell], j \in[1, \ell-n]}$ and $\mu_{T_{k}}(T)=\left(T / T_{k}\right) \oplus T_{k}^{*}$. Set $T_{+}:=\bigoplus_{j ; b_{j k}>0} T_{j}^{\oplus b_{j k}}$ and $T_{-}:=$ $\bigoplus_{j ; b_{j k}<0} T_{j}^{\oplus\left(-b_{j k}\right)}$. Then we have

$$
Y_{T_{k}^{*}} Y_{T_{k}}=q^{-\operatorname{dim}_{\mathbb{C}} \operatorname{Hom}_{\Pi}\left(T_{k}, T_{k}^{*}\right)}\left(q Y_{T_{+}}+Y_{T_{-}}\right) .
$$

Proposition 7.18 (GLS13, Lemma 10.6, Lemma 10.7]). Let $T$ be a basic reachable $\mathcal{C}_{w^{-}}$ maximal rigid module. Then there exists a unique $\mathbb{Q}$-algebra anti-involution $\sigma_{T}^{\prime}$ on $\mathcal{T}_{\mathcal{A}, T}$ such that

$$
q \mapsto q^{-1}, Y_{R} \mapsto q^{-(\underline{\operatorname{dim}} R, \underline{\operatorname{dim}} R) / 2-\operatorname{dim}_{\mathbb{C}} R} Y_{R}
$$

for every direct summand $R$ of a direct sum of copies of $T$. Moreover $\sigma_{T}^{\prime}$ induces $\mathbb{Q}$-algebra anti-involutions $\sigma^{\prime}$ on $\mathscr{A}_{q^{ \pm 1}}\left(\mathcal{C}_{w}\right)$ and $\widetilde{\mathscr{A}}_{q^{ \pm 1}}\left(\mathcal{C}_{w}\right)$, and $\sigma^{\prime}$ does not depend on the choice of a basic reachable $\mathcal{C}_{w}$-maximal rigid module $T$.

Geiß-Leclerc-Schröer showed that a rescaled quantum cluster algebra $\mathscr{A}_{\mathbb{Q}(q)}\left(\mathcal{C}_{w}\right)$ gives an additive categorification of the quantum unipotent subgroup $\mathbf{A}_{q}\left[N_{-}(w)\right]$ as follows.

Proposition 7.19 ([GLS13, Theorem 12.3]). Let $w \in W$ and $\boldsymbol{i}=\left(i_{1}, \ldots, i_{\ell}\right) \in I(w)$. Then there is an isomorphism of $\mathbb{Q}(q)$-algebras $\kappa: \mathbf{A}_{q}\left[N_{-}(w)\right] \rightarrow \mathscr{A}_{\mathbb{Q}(q)}\left(\mathcal{C}_{w}\right)$ given by

$$
D_{s_{i_{1}} \cdots s_{i_{b}} \varpi_{i_{b}}, s_{i_{1}} \cdots s_{i_{a-}} \varpi_{i_{a}}} \mapsto Y_{M[b, a]}
$$

for all $1 \leq a \leq b \leq \ell$ with $i_{a}=i_{b}$. Moreover we have $\sigma^{\prime} \circ \kappa=\kappa \circ \sigma^{\prime}$. Recall Definition 3.11.

By Theorem 4.13, this result also gives an additive categorification of the quantum unipotent cell $\mathbf{A}_{q}\left[N_{-}^{w}\right]$. 
Corollary 7.20. Let $w \in W$ and $\boldsymbol{i}=\left(i_{1}, \ldots, i_{\ell}\right) \in I(w)$. Then there is an isomorphism of $\mathbb{Q}(q)$-algebras $\widetilde{\kappa}: \mathbf{A}_{q}\left[N_{-}^{w}\right] \rightarrow \widetilde{\mathscr{A}_{\mathbb{Q}}(q)}\left(\mathcal{C}_{w}\right)$ given by

$$
\left[D_{s_{i_{1}} \cdots s_{i_{b}} \varpi_{i_{b}}, s_{i_{1}} \cdots s_{i_{a}-} \varpi_{i_{a}}}\right] \mapsto Y_{M[b, a]}
$$

for all $1 \leq a \leq b \leq \ell$ with $i_{a}=i_{b}$. Moreover we have $\sigma^{\prime} \circ \widetilde{\kappa}=\widetilde{\kappa} \circ \sigma^{\prime}$. Recall Definition 4.10 .

The following is the classical counterpart of the results above due to Geiß-Leclerc-Schröer. Note that we explain it as a "specialization" of the results above but it is actually the preceding result of them.

Proposition 7.21 (GLS11, Theorem 3.1, Theorem 3.3]). Let $w \in W$. For every reachable $\Pi$-module $R$ in $\mathcal{C}_{w}$, we have $\varphi_{R} \in \mathbb{C}\left[N_{-}(w)\right]$, and the correspondence

$$
\varphi_{R}\left(\operatorname{resp} .\left[\varphi_{R}\right]\right) \mapsto 1 \otimes Y_{R} .
$$

gives the $\mathbb{C}$-algebra isomorphism from $\mathbb{C}\left[N_{-}(w)\right]$ (resp. $\left.\mathbb{C}\left[N_{-}^{w}\right]\right)$ to $\mathbb{C} \otimes_{\mathcal{A}} \mathscr{A}_{q^{ \pm 1}}\left(\mathcal{C}_{w}\right)$ (resp. $\mathbb{C} \otimes_{\mathcal{A}}$ $\left.\widetilde{\mathscr{A}}_{q^{ \pm 1}}\left(\mathcal{C}_{w}\right)\right)$.

Definition 7.22. Let $T$ be a basic reachable $\mathcal{C}_{w}$-maximal rigid module and $T=T_{1} \oplus \cdots \oplus T_{\ell}$ its

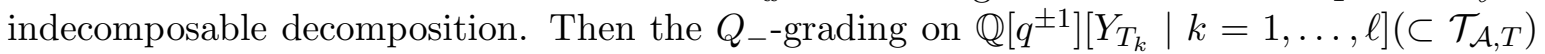
given by wt $Y_{T_{k}}=\underline{\operatorname{dim}} T_{k}$ is extended to the $Q$-grading on $\mathcal{T}_{\mathcal{A}, T}$. A homogeneous element $X \in \mathcal{T}_{\mathcal{A}, T}$ is said to be dual bar invariant if

$$
\sigma_{T}^{\prime}(X)=q^{-(\operatorname{wt} X, \mathrm{wt} X) / 2+(\operatorname{wt} X, \rho)} X,
$$

here recall that $\rho:=\sum_{i \in I} \varpi_{i}$ (Definition 2.1). When $X \in \mathscr{A}_{\mathbb{Q}(q)}\left(\mathcal{C}_{w}\right)\left(\operatorname{resp} . \widetilde{\mathscr{A}}_{\mathbb{Q}(q)}\left(\mathcal{C}_{w}\right)\right)$, the $Q$-grading and the definition of dual bar invariance of homogeneous elements are compatible with the corresponding notions in $\mathbf{A}_{q}\left[N_{-}(w)\right]$ (resp. $\mathbf{A}_{q}\left[N_{-}^{w}\right]$ ) via $\kappa$ (resp. $\widetilde{\kappa}$ ). See Remark 3.12. Note that $Y_{R}$ is dual bar invariant for any reachable $\Pi$-module $R$.

Remark 7.23. Through $\kappa$ (resp. $\widetilde{\kappa}$ ), we can translate also the nontwisted dual bar involution $\sigma$ on $\mathbf{A}_{q}\left[N_{-}(w)\right]$ (resp. $\left.\mathbf{A}_{q}\left[N_{-}^{w}\right]\right)$ into the involution on $\mathscr{A}_{\mathbb{Q}(q)}\left(\mathcal{C}_{w}\right)$ (resp. $\widetilde{\mathscr{A}}_{\mathbb{Q}(q)}\left(\mathcal{C}_{w}\right)$ ). Then this involution coincides with the twisted bar involution in the sense of [BZ05, Section 6] if we take a grading datum $\Sigma=\left(\sigma_{i j}\right)_{i, j \in[1, \ell]}$ associated with $T$ in Definition 7.22 as $\sigma_{i j}=$ $-\left(\underline{\operatorname{dim}} T_{i}, \underline{\operatorname{dim}} T_{j}\right)$ for $i, j \in[1, \ell]$ (see [BZ05, Definition 6.5] for the definition of the notion of grading).

Geiß-Leclerc-Schröer also obtained an additive categorification of the twist automorphism $\eta_{w}^{*}$ on the coordinate algebra $\mathbb{C}\left[N_{-}^{w}\right]$ of a unipotent cell $N_{-}^{w}$ in non-quantum settings. Here the image of $\varphi_{X} \in \mathbb{C}\left[N_{-}\right]$under the restriction map $\mathbb{C}\left[N_{-}\right] \rightarrow \mathbb{C}\left[N_{-}^{w}\right]$ is denoted by $\left[\varphi_{X}\right] \in$ $\mathbb{C}\left[N_{-}^{w}\right]$.

Proposition 7.24 ([GLS12, Theorem 6]). Let $w \in W$. Then for every $X \in \mathcal{C}_{w}$ we have

$$
\eta_{w}^{*}\left(\left[\varphi_{X}\right]\right)=\frac{\left[\varphi_{\Omega_{w}^{-1}(X)}\right]}{\left[\varphi_{I(X)}\right]} .
$$

7.3. Quantum twist automorphisms and the quantum algebra structures. Our main result in this subsection is the following quantum analogue of Proposition 7.24, Recall Proposition 7.15 . 
Theorem 7.25. Let $w \in W, T$ a basic reachable $\mathcal{C}_{w}$-maximal rigid module, and $T=T_{1} \oplus \cdots \oplus$ $T_{\ell}$ its indecomposable decomposition. Through $\widetilde{\kappa}$ in Corollary 7 .20, we regard the quantum twist map $\eta_{w, q}$ as an algebra automorphism on $\widetilde{\mathscr{A}}_{\mathbb{Q}(q)}\left(\mathcal{C}_{w}\right)$. Then, for every direct summand $R$ of a direct sum of copies of $T$ (that is, every reachable $\Pi$-module $R$ in $\mathcal{C}_{w}$ ), we have

$$
\eta_{w, q}\left(Y_{R}\right)=q^{\sum_{i \in I_{w}} \lambda_{i} \operatorname{dim}_{\mathbb{C}} \epsilon_{i} \cdot R} Y_{I(R)}^{-1} Y_{\Omega_{w}^{-1}(R)} .
$$

here we write $I(R)=\bigoplus_{i \in I_{w}} T_{\ell-n+i}^{\oplus \lambda_{i}}$.

As a corollary of the above result, we obtain the following.

Corollary 7.26. Let $R$ be a reachable $\Pi$-module in $\mathcal{C}_{w}$. Then $\kappa^{-1}\left(Y_{R}\right) \in \mathbf{B}^{\mathrm{up}} \cap \mathbf{A}_{q}\left[N_{-}(w)\right]$ if and only if $\kappa^{-1}\left(Y_{\Omega_{w}^{-1}(R)}\right) \in \mathbf{B}^{\mathrm{up}} \cap \mathbf{A}_{q}\left[N_{-}(w)\right]$.

Before proving Theorem 7.25 , we show its corollary.

Proof. By Theorem 6.1 and 7.25, $\kappa^{-1}\left(Y_{R}\right) \in \mathbf{B}^{\text {up }}$ if and only if $\widetilde{\kappa}^{-1}\left(q^{\sum_{i \in I_{w}} \lambda_{i} \operatorname{dim}_{\mathbb{C}} \epsilon_{i} \cdot R} Y_{I(R)}^{-1} Y_{\Omega_{w}^{-1}(R)}\right) \in$ $\widetilde{\mathbf{B}}^{\mathrm{up}, w}$. By Theorem 6.1 and the dual bar invariance of $Y_{R}$, the element $q^{\sum_{i \in I_{w}} \lambda_{i} \operatorname{dim}_{\mathbb{C}} \epsilon_{i} \cdot R} Y_{I(R)}^{-1} Y_{\Omega_{w}^{-1}(R)}$ is also dual bar invariant. Combining this fact with the definition of $\widetilde{\mathbf{B}}^{\text {up }, w}=\iota_{w}\left(\widetilde{\mathbf{B}}^{\text {up }}(w)\right)$ and the dual bar invariance of $Y_{\Omega_{w}^{-1}(R)}$, we have $\widetilde{\kappa}^{-1}\left(q^{\sum_{i \in I_{w}} \lambda_{i} \operatorname{dim}_{\mathbb{C}} \epsilon_{i} \cdot R} Y_{I(R)}^{-1} Y_{\Omega_{w}^{-1}(R)}\right) \in \widetilde{\mathbf{B}}^{\text {up }, w}$ if and only if $\kappa^{-1}\left(Y_{\Omega_{w}^{-1}(R)}\right) \in \mathbf{B}^{\text {up }}$.

Remark 7.27. Kang-Kashiwara-Kim-Oh KKKO18 have shown that all (rescaled) quantum cluster monomials belong to $\mathbf{B}^{\text {up }}$ by using the categorification via representations of quiver Hecke algebras. (See [KKKO18, Introduction] for several results of this direction before [KKKO18].) Hence we have already known that $Y_{R}$ is an element of $\mathbf{B}^{\text {up }}$ for an arbitrary reachable $\Pi$-module in $\mathcal{C}_{w}$. However there is now no proof of this strong result through the additive categorification above. Therefore it would be interesting to determine the quantum monomials in $\mathbf{B}^{\mathrm{up}}$ which are obtained from Corollary 7.26 and, for example, $\left(Y_{V_{\boldsymbol{i}}}\right)^{\boldsymbol{a}}$ for $\boldsymbol{a} \in$ $\mathbb{Z}_{>0}^{\ell(w)}$ and $\boldsymbol{i} \in I(w)$. Actually, it is easy to show that $\left(Y_{V_{\boldsymbol{i}}}\right)^{\boldsymbol{a}} \in \mathbf{B}^{\text {up }}$ by Proposition 4.1. Moreover it is unclear whether a quantum twist automorphism $\eta_{w, q}$ is categorified by using finite dimensional representations of quiver Hecke algebras. In particular, we do not know that quantum twist automorphisms preserve the basis coming from the simple modules of quiver Hecke algebras.

The rest of this subsection is devoted to the proof of Theorem 7.25. In this proof, we essentially use Geiß-Leclerc-Schröer's theory.

Lemma 7.28. Let $T$ be a basic reachable $\mathcal{C}_{w}$-maximal rigid module and $T=T_{1} \oplus \cdots \oplus T_{\ell}$ its indecomposable decomposition. Take $\left(a_{1}, \ldots, a_{\ell}\right) \in \mathbb{Z}^{\ell}$. Then there exists a unique integer $m$ such that $q^{m} Y_{T_{1}}^{a_{1}} \cdots Y_{T_{\ell}}^{a_{\ell}}$ is dual bar invariant in $\mathcal{T}_{\mathcal{A}, T}$.

Proof. We have

$$
\begin{aligned}
\sigma_{T}^{\prime}\left(q^{m} Y_{T_{1}}^{a_{1}} \cdots Y_{T_{\ell}}^{a_{\ell}}\right) & =q^{-m} \sigma_{T}^{\prime}\left(Y_{T_{\ell}}\right)^{a_{\ell}} \cdots \sigma_{T}^{\prime}\left(Y_{T_{1}}\right)^{a_{1}} \\
& =q^{\left.-m+\sum_{i \in[1, \ell]} a_{i}\left(-\underline{\operatorname{dim}} T_{i}, \underline{\operatorname{dim}} T_{i}\right) / 2+\left(\underline{\operatorname{dim}} T_{i}, \rho\right)\right)} Y_{T_{\ell}}^{a_{\ell}} \cdots Y_{T_{1}}^{a_{1}} \\
& =q^{-m+\sum_{i \in[1, \ell]} a_{i}\left(-\left(\underline{\operatorname{dim}} T_{i}, \underline{\operatorname{dim}} T_{i}\right) / 2+\left(\underline{\operatorname{dim}} T_{i}, \rho\right)\right)-\sum_{i<j} a_{i} a_{j} \lambda_{i j}} Y_{T_{1}}^{a_{1}} \cdots Y_{T_{\ell}}^{a_{\ell}} .
\end{aligned}
$$


Here we write $\Lambda_{T}=\left(\lambda_{i j}\right)_{i, j \in[1, \ell]}$. Therefore $q^{m} Y_{T_{1}}^{a_{1}} \cdots Y_{T_{\ell}}^{a_{\ell}}$ is dual bar invariant if and only if

$$
\begin{aligned}
& m-\sum_{i \in[1, \ell]} a_{i}^{2}\left(\underline{\operatorname{dim}} T_{i}, \underline{\operatorname{dim}} T_{i}\right) / 2-\sum_{i<j} a_{i} a_{j}\left(\underline{\operatorname{dim}} T_{i}, \underline{\operatorname{dim}} T_{j}\right)+\sum_{i \in[1, \ell]} a_{i}\left(\underline{\operatorname{dim}} T_{i}, \rho\right) \\
& =-m+\sum_{i \in[1, \ell]} a_{i}\left(-\left(\underline{\operatorname{dim}} T_{i}, \underline{\operatorname{dim}} T_{i}\right) / 2+\left(\underline{\operatorname{dim}} T_{i}, \rho\right)\right)-\sum_{i<j} a_{i} a_{j} \lambda_{i j} .
\end{aligned}
$$

By Remark 7.11, this is equivalent to

$$
2 m=\sum_{i \in[1, \ell]} a_{i}\left(a_{i}-1\right)\left(\underline{\operatorname{dim}} T_{i}, \underline{\operatorname{dim}} T_{i}\right) / 2+2 \sum_{i<j} a_{i} a_{j} \operatorname{dim}_{\mathbb{C}} \operatorname{Hom}_{\Pi}\left(T_{j}, T_{i}\right) .
$$

The right-hand side is an element of $2 \mathbb{Z}$. Therefore we can take an integer $m \in \mathbb{Z}$ uniquely which satisfies this equality.

Remark 7.29. For $\left(a_{1}, \ldots, a_{\ell}\right) \in \mathbb{Z}_{\geq 0}^{\ell}$, the unique dual bar invariant element in $\left\{q^{m} Y_{T_{1}}^{a_{1}} \cdots Y_{T_{\ell}}^{a_{\ell}} \mid\right.$ $m \in \mathbb{Z}\}$ is $Y_{\bigoplus_{i \in[1, \ell]}} T_{i}^{\oplus a_{i}}$.

Lemma 7.30. With the notation in Theorem 7.25, $q^{\sum_{i \in I_{w}} \lambda_{i} \operatorname{dim}_{\mathbb{C}} \epsilon_{i} \cdot R_{Y}} Y_{I(R)}^{-1} Y_{\Omega_{w}^{-1}(R)}$ is dual bar invariant.

Proof. By Proposition 7.19,

$$
\kappa^{-1}\left(Y_{I(R)}\right)=D_{w \lambda, \lambda},
$$

here $\lambda:=\sum_{i \in I_{w}} \lambda_{i} \varpi_{i}$. Hence, by Proposition 4.2, we have

$$
\begin{aligned}
\kappa^{-1}\left(Y_{I(R)} Y_{\Omega_{w}^{-1}(R)}\right) & =D_{w \lambda, \lambda} \kappa^{-1}\left(Y_{\Omega_{w}^{-1}(R)}\right) \\
& =q^{\left(\lambda+w \lambda, \underline{\operatorname{dim}} \Omega_{w}^{-1}(R)\right)} \kappa^{-1}\left(Y_{\Omega_{w}^{-1}(R)}\right) D_{w \lambda, \lambda} \\
& =q^{\left(\lambda+w \lambda, \underline{\operatorname{dim}} \Omega_{w}^{-1}(R)\right)} \kappa^{-1}\left(Y_{\Omega_{w}^{-1}(R)} Y_{I(R)}\right) .
\end{aligned}
$$

By the way, $\underline{\operatorname{dim}} \Omega_{w}^{-1}(R)=\underline{\operatorname{dim}} I(R)-\underline{\operatorname{dim}} R=w \lambda-\lambda-\underline{\operatorname{dim}} R$. Hence $\left(\lambda+w \lambda, \underline{\operatorname{dim}} \Omega_{w}^{-1}(R)\right)=$ $-(\lambda+w \lambda, \underline{\operatorname{dim} R})$. Therefore

$$
Y_{I(R)}^{-1} Y_{\Omega_{w}^{-1}(R)}=q^{(\lambda+w \lambda, \underline{\operatorname{dim}} R)} Y_{\Omega_{w}^{-1}(R)} Y_{I(R)}^{-1}
$$

Note that $\sum_{i \in I_{w}} \lambda_{i} \operatorname{dim}_{\mathbb{C}} \epsilon_{i} . R=-(\lambda, \underline{\operatorname{dim}} R)$. We have

$$
\begin{aligned}
& \left.q \underline{(\operatorname{dim}} \Omega_{w}^{-1}(R)-\underline{\operatorname{dim}} I(R), \underline{\operatorname{dim}} \Omega_{w}^{-1}(R)-\underline{\operatorname{dim}} I(R)\right) / 2-\left(\underline{\operatorname{dim}} \Omega_{w}^{-1}(R)-\underline{\operatorname{dim}} I(R), \rho\right) \sigma_{T}^{\prime}\left(q^{-(\lambda, \underline{\operatorname{dim}} R)} Y_{I(R)}^{-1} Y_{\Omega_{w}^{-1}(R)}\right) \\
& =q \underline{(\operatorname{dim}} R, \underline{\operatorname{dim}} R) / 2+(\underline{\operatorname{dim}} R, \rho) \sigma_{T}^{\prime}\left(q^{(w \lambda, \underline{\operatorname{dim}} R)} Y_{\Omega_{w}^{-1}(R)} Y_{I(R)}^{-1}\right)
\end{aligned}
$$

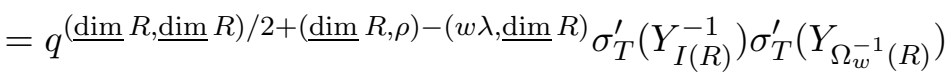

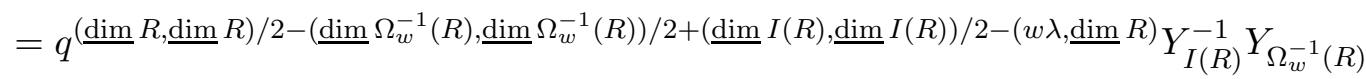

$$
\begin{aligned}
& =q^{(\underline{\operatorname{dim}} I(R), \underline{\operatorname{dim}} R)-(w \lambda, \underline{\operatorname{dim}} R)} Y_{I(R)}^{-1} Y_{\Omega_{w}^{-1}(R)} \\
& =q^{-(\lambda, \underline{\operatorname{dim}} R)} Y_{I(R)}^{-1} Y_{\Omega_{w}^{-1}(R)} .
\end{aligned}
$$

This competes the proof.

Lemma 7.31. Let $T$ be a basic reachable $\mathcal{C}_{w}$-maximal rigid module and $T=T_{1} \oplus \cdots \oplus T_{\ell}$ its indecomposable decomposition. Then the equality (7.3) with $R=T_{k}$ holds for all $k=1, \ldots, \ell$ if and only if the one with $R=T_{1}^{\oplus a_{1}} \oplus \cdots \oplus T_{\ell}^{\oplus a_{\ell}}$ holds for all $\left(a_{1}, \ldots, a_{\ell}\right) \in \mathbb{Z}_{\geq 0}^{\ell}$. 
Proof. The latter obviously implies the former. Suppose that the equality (7.3) holds for $R=T_{k}, k=1, \ldots, \ell$. Write

$$
\eta_{w, q}\left(Y_{T_{k}}\right)=q^{m_{k}} Y_{I\left(T_{k}\right)}^{-1} Y_{\Omega_{w}^{-1}\left(T_{k}\right)}, m_{k} \in \mathbb{Z}
$$

for $k=1, \ldots, \ell$. Set $R=T_{1}^{\oplus a_{1}} \oplus \cdots \oplus T_{\ell}^{\oplus a_{\ell}}$ for $\left(a_{1}, \ldots, a_{\ell}\right) \in \mathbb{Z}_{\geq 0}^{\ell}$. Note that $I(R)=I\left(T_{1}\right)^{\oplus a_{1}} \oplus$ $\cdots \oplus I\left(T_{\ell}\right)^{\oplus a_{\ell}}$ and $\Omega_{w}^{-1}(R)=\Omega_{w}^{-1}\left(T_{1}\right)^{\oplus a_{1}} \oplus \cdots \oplus \Omega_{w}^{-1}\left(T_{\ell}\right)^{\oplus a_{\ell}}$. (Actually $I\left(T_{\ell-n+i}\right)=T_{\ell-n+i}$ and $\Omega_{w}^{-1}\left(T_{\ell-n+i}\right)=0$ for $i \in I_{w}$.) There exist unique $A_{1}, A_{2}, A_{3} \in \mathbb{Z}$ such that the following hold:

$$
\begin{aligned}
\eta_{w, q}\left(Y_{R}\right) & =q^{A_{1}} \eta_{w, q}\left(Y_{T_{1}}^{a_{1}} \cdots Y_{T_{\ell}}^{a_{\ell}}\right) \\
& =q^{A_{1}}\left(q^{m_{1}} Y_{I\left(T_{1}\right)}^{-1} Y_{\Omega_{w}^{-1}\left(T_{1}\right)}\right)^{a_{1}} \cdots\left(q^{m_{\ell}} Y_{I\left(T_{\ell}\right)}^{-1} Y_{\Omega_{w}^{-1}\left(T_{\ell}\right)}\right)^{a_{\ell}} \\
& =q^{A_{2}}\left(Y_{I\left(T_{1}\right)}^{a_{1}} \cdots Y_{I\left(T_{\ell}\right)}^{a_{\ell}}\right)^{-1} Y_{\Omega_{w}^{-1}\left(T_{1}\right)}^{a_{1}} \cdots Y_{\Omega_{w}^{-1}\left(T_{\ell}\right)}^{a_{\ell}} \\
& =q^{A_{3}} Y_{I(R)}^{-1} Y_{\Omega_{w}^{-1}(R)} .
\end{aligned}
$$

Moreover $\eta_{w, q}\left(Y_{R}\right)$ is dual bar invariant because of the dual bar invariance of $Y_{R}$ and Theorem 6.1. Hence, by Lemma 7.28 and Lemma 7.30, the equality (7.3) also holds for $R$.

Proof of Theorem 7.25. Recall that we always assume that $T_{\ell-n+i}$ is a $\mathcal{C}_{w}$-projective-injective module with socle $S_{i}$ for all $i \in I_{w}=[1, n]$, in particular, the isomorphism class of $T_{\ell-n+i}$ does not depend on the choice of $T$. From now on, we identify $\widetilde{\mathscr{A}_{\mathbb{Q}(q)}}\left(\mathcal{C}_{w}\right)$ with $\mathbf{A}_{q}\left[N_{-}^{w}\right]$ via $\widetilde{\kappa}$. First we consider the case that $R$ in the statement of Theorem 7.25 is equal to $T_{\ell-n+i}$ for $i \in I_{w}$. Then

$$
\begin{aligned}
\eta_{w, q}\left(Y_{T_{\ell-n+i}}\right) & =\eta_{w, q}\left(\left[D_{w \varpi_{i}, \varpi_{i}}\right]\right) \\
& =q^{-\left(\varpi_{i}, w \varpi_{i}-\varpi_{i}\right)}\left[D_{w \varpi_{i}, \varpi_{i}}\right]^{-1} \\
& =q^{\operatorname{dim}_{\mathbb{C}} \epsilon_{i} \cdot T_{\ell-n+i}} Y_{T_{\ell-n+i}}^{-1},
\end{aligned}
$$

which is the desired equality in this case since $I\left(T_{\ell-n+i}\right)=T_{\ell-n+i}$ and $\Omega_{w}^{-1}\left(T_{\ell-n+i}\right)=0$.

Let $i \in I(w)$. Henceforth, we will prove the theorem by induction on the minimal length of sequences of mutations which we need to obtain $T$ from $V_{\boldsymbol{i}}$. We begin with the case that $R=$ $V_{\boldsymbol{i}, k}$ for some $k \in[1, \ell]$ with $k^{+} \neq \ell+1$. Then $I\left(V_{i, k}\right)=V_{\boldsymbol{i}, k^{\max }}$ and $\Omega_{w}^{-1}\left(V_{\boldsymbol{i}, k}\right)=M_{\boldsymbol{i}}\left[k^{\max }, k^{+}\right]$. Therefore we have

$$
\begin{aligned}
\eta_{w, q}\left(Y_{V_{\boldsymbol{i}, k}}\right) & =\eta_{w, q}\left(\left[D_{s_{i_{1}} \cdots s_{i_{k}} \varpi_{i_{k}}, \varpi_{i_{k}}}\right]\right) \\
& =q^{-\left(\varpi_{i_{k}}, s_{i_{1}} \cdots s_{i_{k}} \varpi_{i_{k}}-\varpi_{i_{k}}\right)}\left[D_{w \varpi_{i_{k}}, \varpi_{i_{k}}}\right]^{-1}\left[D_{u_{w \varpi_{i_{k}}}, s_{i_{1}} \cdots s_{i_{k}} \varpi_{i_{k}}}\right] \\
& =q^{-\left(\varpi_{i_{k}}, \underline{\operatorname{dim}} V_{\boldsymbol{i}, k}\right)} Y_{V_{\boldsymbol{i}, k} \max }^{-1} Y_{M_{\boldsymbol{i}}\left[k^{\max }, k^{+}\right]} \\
& =q^{\operatorname{dim}_{\mathbb{C}} \epsilon_{i} . V_{\boldsymbol{i}, k}} Y_{I\left(V_{\boldsymbol{i}, k}\right)}^{-1} Y_{\Omega_{w}^{-1}\left(V_{\boldsymbol{i}, k}\right)} .
\end{aligned}
$$

Hence, by Lemma 7.31, the equality (7.3) holds when $R=V_{\boldsymbol{i}}$. Next, suppose that the equality (7.3) holds for $R=T_{1}^{\oplus a_{1}} \oplus \cdots \oplus T_{\ell}^{\oplus a_{\ell}}$, where $T=T_{1} \oplus \cdots \oplus T_{\ell}$ is a basic reachable $\mathcal{C}_{w^{-m a x i m a l}}$ rigid module. Let $k \in[1, \ell-n]$ and write $\mu_{T_{k}}(T)=\left(T / T_{k}\right) \oplus T_{k}^{*}, I\left(T_{k}^{*}\right)=\bigoplus_{i \in I_{w}} T_{\ell-n+i}^{\oplus \lambda_{i}}$. Then, by Lemma 7.31, it remains to prove the following equality:

$$
\eta_{w, q}\left(Y_{T_{k}^{*}}\right)=q^{\sum_{i \in I_{w}} \lambda_{i} \operatorname{dim}_{\mathbb{C}} \epsilon_{i} \cdot T_{k}^{*}} Y_{I\left(T_{k}^{*}\right)}^{-1} Y_{\Omega_{w}^{-1}\left(T_{k}^{*}\right)} .
$$


Write $\tilde{B}_{T}=\left(b_{i j}\right)_{i \in[1, \ell], j \in[1, \ell-n]}$. Set $T_{+}:=\bigoplus_{j ; b_{j k}>0} T_{j}^{\oplus b_{j k}}$ and $T_{-}:=\bigoplus_{j ; b_{j k}<0} T_{j}^{\oplus\left(-b_{j k}\right)}$. By (7.1), Proposition [7.7 (2) and Proposition 7.24, we have

$$
\eta_{w}^{*}\left(\left[\varphi_{T_{k}} \varphi_{T_{k}^{*}}\right]\right)=\eta_{w}^{*}\left(\left[\varphi_{T_{+}}\right]+\left[\varphi_{T_{-}}\right]\right)=\frac{\left[\varphi_{\Omega_{w}^{-1}\left(T_{+}\right)}\right]}{\left[\varphi_{I\left(T_{+}\right)}\right]}+\frac{\left[\varphi_{\Omega_{w}^{-1}\left(T_{-}\right)}\right]}{\left[\varphi_{I\left(T_{-}\right)}\right]},
$$

and

$$
\eta_{w}^{*}\left(\left[\varphi_{T_{k}} \varphi_{T_{k}^{*}}\right]\right)=\frac{\left[\varphi_{\Omega_{w}^{-1}\left(T_{k}\right)}\right]}{\left[\varphi_{I\left(T_{k}\right)}\right]} \cdot \frac{\left[\varphi_{\Omega_{w}^{-1}\left(T_{k}^{*}\right)}\right]}{\left[\varphi_{I\left(T_{k}^{*}\right)}\right]}=\frac{\left[\varphi_{\Omega_{w}^{-1}\left(T_{k}\right)} \varphi_{\Omega_{w}^{-1}\left(T_{k}^{*}\right)}\right]}{\left[\varphi_{I\left(T_{k} \oplus T_{k}^{*}\right)}\right]} .
$$

Therefore ,

$$
\left[\varphi_{\Omega_{w}^{-1}\left(T_{k}\right)} \varphi_{\Omega_{w}^{-1}\left(T_{k}^{*}\right)}\right]=\left[\varphi_{I\left(T_{k} \oplus T_{k}^{*}\right)}\right]\left(\frac{\left[\varphi_{\Omega_{w}^{-1}\left(T_{+}\right)}\right]}{\left[\varphi_{I\left(T_{+}\right)}\right]}+\frac{\left[\varphi_{\Omega_{w}^{-1}\left(T_{-}\right)}\right]}{\left[\varphi_{I\left(T_{-}\right)}\right]}\right) .
$$

By Proposition 17.15, $T^{\prime}:=\Omega_{w}^{-1}(T) \oplus \bigoplus_{i \in I_{w}} T_{\ell-n+i}$ is a basic reachable $\mathcal{C}_{w}$-maximal rigid module; hence there exists a bijection $[1, \ell-n] \rightarrow[1, \ell-n], j \mapsto j^{*}$ such that $T_{j^{*}}^{\prime}=\Omega_{w}^{-1}\left(T_{j}\right)$. Moreover we have

$$
\mu_{T_{k^{*}}^{\prime}}\left(T^{\prime}\right)=\left(T^{\prime} / T_{k^{*}}^{\prime}\right) \oplus \Omega_{w}^{-1}\left(T_{k}^{*}\right)
$$

Write $\tilde{B}_{T^{\prime}}=\left(b_{i j}^{\prime}\right)_{i \in[1, \ell], j \in[1, \ell-n]}$ and $\left(T_{k^{*}}^{\prime}\right)^{*}:=\Omega_{w}^{-1}\left(T_{k}^{*}\right)$. Set $T_{+}^{\prime}:=\bigoplus_{j ; b_{j^{*} k^{*}}^{\prime}>0}\left(T_{j^{*}}^{\prime}\right)^{\oplus b_{j^{*} k^{*}}^{\prime} \text { and }}$ $T_{-}^{\prime}:=\bigoplus_{j ; b_{j^{*} k^{*}}^{\prime}<0}\left(T_{j^{*}}^{\prime}\right)^{\oplus\left(-b_{j^{*} k^{*}}^{\prime}\right)}$. Then, by (7.1) and (7.5), we have

$$
\left[\varphi_{I\left(T_{k} \oplus T_{k}^{*}\right)}\right]\left(\frac{\left[\varphi_{\Omega_{w}^{-1}\left(T_{+}\right)}\right]}{\left[\varphi_{I\left(T_{+}\right)}\right]}+\frac{\left[\varphi_{\Omega_{w}^{-1}\left(T_{-}\right)}\right]}{\left[\varphi_{I\left(T_{-}\right)}\right]}\right)=\left[\varphi_{T_{+}^{\prime}}\right]+\left[\varphi_{T_{-}^{\prime}}\right] .
$$

Note that all $\Pi$-modules appearing in the equality (7.6) are direct summands of a direct sum of copies of $T^{\prime}$. Therefore, by Proposition $7.7(2)$, we have $I\left(T_{k} \oplus T_{k}^{*}\right)=I\left(T_{+}\right) \oplus I_{+}=$ $I\left(T_{-}\right) \oplus I_{-}$for some $\mathcal{C}_{w}$-projective-injective modules $I_{+}, I_{-}$, and

$$
\left\{I_{+} \oplus \Omega_{w}^{-1}\left(T_{+}\right), I_{-} \oplus \Omega_{w}^{-1}\left(T_{-}\right)\right\}=\left\{T_{+}^{\prime}, T_{-}^{\prime}\right\}
$$

By the way, we recall our assumption that the equality (7.3) holds for $R=T_{1}^{\oplus a_{1}} \oplus \cdots \oplus T_{\ell}^{\oplus a_{\ell}}$. By Proposition 7.17 and our assumption, there exist unique $A_{1}, A_{1}^{\prime}, A_{2}, A_{2}^{\prime}, A_{3} \in \mathbb{Z}$ such that

$$
\begin{aligned}
\eta_{w, q}\left(Y_{T_{k}} Y_{T_{k}^{*}}\right) & =\eta_{w, q}\left(q^{A_{1}} Y_{T_{+}}+q^{A_{2}} Y_{T_{-}}\right) \\
& =q^{A_{1}^{\prime}} Y_{I\left(T_{+}\right)}^{-1} Y_{\Omega_{w}^{-1}\left(T_{+}\right)}+q^{A_{2}^{\prime}} Y_{I\left(T_{-}\right)}^{-1} Y_{\Omega_{w}^{-1}\left(T_{-}\right)}
\end{aligned}
$$

and

$$
\eta_{w, q}\left(Y_{T_{k}} Y_{T_{k}^{*}}\right)=q^{A_{3}} Y_{I\left(T_{k}\right)}^{-1} Y_{T_{k^{*}}^{\prime}} \eta_{w, q}\left(Y_{T_{k}^{*}}\right) .
$$

These equalities together with (7.7) imply that there exist unique $A^{\prime}, A_{1}^{\prime \prime}, A_{2}^{\prime \prime} \in \mathbb{Z}$ such that

$$
\begin{aligned}
\eta_{w, q}\left(Y_{T_{k}^{*}}\right) & =q^{-A_{3}} Y_{T_{k^{*}}^{\prime}}^{-1} Y_{I\left(T_{k}\right)}\left(q^{A_{1}^{\prime}} Y_{I\left(T_{+}\right)}^{-1} Y_{\Omega_{w}^{-1}\left(T_{+}\right)}+q^{A_{2}^{\prime}} Y_{I\left(T_{-}\right)}^{-1} Y_{\Omega_{w}^{-1}\left(T_{-}\right)}\right) \\
& =q^{A^{\prime}} Y_{T_{k^{*}}^{\prime}}^{-1} Y_{I\left(T_{k}^{*}\right)}^{-1} Y_{I\left(T_{k} \oplus T_{k}^{*}\right)}\left(q^{A_{1}^{\prime}} Y_{I\left(T_{+}\right)}^{-1} Y_{\Omega_{w}^{-1}\left(T_{+}\right)}+q^{A_{2}^{\prime}} Y_{I\left(T_{-}\right)}^{-1} Y_{\Omega_{w}^{-1}\left(T_{-}\right)}\right) \\
& =Y_{I\left(T_{k}^{*}\right)}^{-1} Y_{T_{k^{*}}^{\prime}}^{-1}\left(q^{A_{1}^{\prime \prime}} Y_{T_{+}^{\prime}}+q^{A_{2}^{\prime \prime}} Y_{T_{-}^{\prime}}\right) .
\end{aligned}
$$

Note that all rescaled quantum cluster monomials appearing in the rightmost hand side of the equality above are monomials of the based quantum torus $\mathcal{T}_{\mathcal{A}, T^{\prime}}$. Moreover, $\eta_{w, q}\left(Y_{T_{k}^{*}}\right)$ 
is dual bar invariant because, by Theorem 6.1, the quantum twist automorphism $\eta_{w, q}$ preserves dual bar invariance property of elements of $\widetilde{\mathscr{A}}_{\mathbb{Q}(q)}\left(\mathcal{C}_{w}\right)$ (recall Definition 7.22). Hence $q^{A_{1}^{\prime \prime}} Y_{I\left(T_{k}^{*}\right)}^{-1} Y_{T_{k^{*}}^{\prime}}^{-1} Y_{T_{+}^{\prime}}$ and $q^{A_{2}^{\prime \prime}} Y_{I\left(T_{k}^{*}\right)}^{-1} Y_{T_{k^{*}}^{\prime}}^{-1} Y_{T_{-}^{\prime}}$ are dual bar invariant elements of $\mathcal{T}_{\mathcal{A}, T^{\prime}}$. By Lemma 7.28. $A_{1}^{\prime \prime}$ and $A_{2}^{\prime \prime}$ are uniquely determined by this property. On the other hand, by Proposition 7.17, $q^{\sum_{i \in I_{w}} \lambda_{i} \operatorname{dim}_{\mathbb{C}} \epsilon_{i} \cdot T_{k}^{*}} Y_{I\left(T_{k}^{*}\right)}^{-1} Y_{\left(T_{k^{*}}^{\prime}\right)}$ is of the following form as an element of $\mathcal{T}_{q^{ \pm 1}, T^{\prime}}$ :

$$
Y_{I\left(T_{k}^{*}\right)}^{-1} Y_{T_{k^{*}}^{\prime}}^{-1}\left(q^{M_{1}} Y_{T_{+}^{\prime}}+q^{M_{2}} Y_{T_{-}^{\prime}}\right), M_{1}, M_{2} \in \mathbb{Z}
$$

Moreover, by Lemma 7.30, $q^{\sum_{i \in I_{w}} \lambda_{i} \operatorname{dim}_{\mathbb{C}} \epsilon_{i} \cdot T_{k}^{*}} Y_{I\left(T_{k}^{*}\right)}^{-1} Y_{\left(T_{k^{*}}^{\prime}\right)^{*}}=q^{\sum_{i \in I_{w}} \lambda_{i} \operatorname{dim}_{\mathbb{C}} \epsilon_{i} \cdot T_{k}^{*}} Y_{I\left(T_{k}^{*}\right)}^{-1} Y_{\Omega_{w}^{-1}\left(T_{k}^{*}\right)}$ is dual bar invariant. Hence, by the argument above, $M_{1}=A_{1}^{\prime}$ and $M_{2}=A_{2}^{\prime \prime}$. Therefore we obtain

$$
\eta_{w, q}\left(Y_{T_{k}^{*}}\right)=q^{\sum_{i \in I_{w}} \lambda_{i} \operatorname{dim}_{\mathbb{C}} \epsilon_{i} \cdot T_{k}^{*}} Y_{I\left(T_{k}^{*}\right)}^{-1} Y_{\Omega_{w}^{-1}\left(T_{k}^{*}\right)},
$$

which completes the proof.

\section{Finite type CASES : 6-PERIODICITY}

Since the map $\eta_{q, w}$ is an automorphism, we can apply it repeatedly. In this section, we show the "6-periodicity" of specific quantum twist automorphisms. Assume that $\mathfrak{g}$ is a finite dimensional Lie algebra, and let $w_{0}$ be the longest element of $W$.

Theorem 8.1. For a homogeneous element $x \in \mathbf{A}_{q}\left[N_{-}^{w_{0}}\right]$, we have

$$
\eta_{w_{0}, q}^{6}(x)=q^{\left(\mathrm{wt} x+w_{0} \operatorname{wt} x, \mathrm{wt} x\right)} D_{w_{0},-\operatorname{wt} x-w_{0} \mathrm{wt} x} x .
$$

Remark 8.2. When the action of $w_{0}$ on $P$ is given by $\mu \mapsto-\mu$, the theorem above states that $\eta_{w_{0}, q}^{6}=\mathrm{id}$. Hence $\eta_{w_{0}, q}$ is "really" periodic. If $\mathfrak{g}$ is simple, then this condition is satisfied in the case that $\mathfrak{g}$ is of type $\mathrm{B}_{n}, \mathrm{C}_{n}, \mathrm{D}_{2 n}$ for $n \in \mathbb{Z}_{>0}$ and $\mathrm{E}_{7}, \mathrm{E}_{8}, \mathrm{~F}_{4}, \mathrm{G}_{2}$. See Hum90, Section 3.7 .

When $\mathfrak{g}$ is symmetric, such periodicity is also explained by Geiß-Leclerc-Schröer's additive categorification of twist automorphisms (see Section 7). The periodicity corresponds to the well-known 6-periodicity of syzygy functors [AR96, ES98], that is, the property that $\left(\Omega_{w_{0}}^{-1}\right)^{6}(M) \simeq M$ for an indecomposable non-projective-injective module $M$ of $\Pi$ in the notation of Section 7 .

We can consider the similar periodicity problems for every $w \in W$. It would be interesting to find the necessary and sufficient condition on $w \in W$ for periodicity. Since quantum twist automorphisms restrict to permutations on dual canonical bases, the periodicity of a quantum twist automorphism $\eta_{w, q}$ is equivalent to the periodicity of a (non-quantum) twist automorphism $\eta_{w}$. See also Remark 8.4 below.

Lemma 8.3. Let $\lambda \in P_{+}$. Take $u, u^{\prime} \in V(\lambda)$ such that $D_{u, u^{\prime}}=G^{\text {up }}(\tilde{b})$ for some $\tilde{b} \in \mathscr{B}(\infty)$. Then, for $i \in I$,

$$
\varepsilon_{i}(\tilde{b})=\max \left\{k \in \mathbb{Z}_{\geq 0} \mid D_{e_{i}^{k} \cdot u, u^{\prime}} \neq 0\right\} \quad \varepsilon_{i}^{*}(\tilde{b})=\max \left\{k \in \mathbb{Z}_{\geq 0} \mid D_{u, f_{i}^{k} \cdot u^{\prime}} \neq 0\right\} .
$$

In particular,

$$
\varepsilon_{i}\left(\bar{\jmath}_{\lambda}(b)\right)=\varepsilon_{i}(b) \quad \varepsilon_{i}\left(\bar{\jmath}_{w_{0} \lambda}^{\vee}(b)\right)=\varphi_{i}(b)\left(=\varepsilon_{i}(b)+\left\langle h_{i}, \text { wt } b\right\rangle\right) .
$$


Proof. By Proposition 3.22 ,

$$
\varepsilon_{i}(\tilde{b})=\max \left\{k \in \mathbb{Z}_{\geq 0} \mid\left(e_{i}^{\prime}\right)^{k}\left(D_{u, u^{\prime}}\right) \neq 0\right\} .
$$

For $k \in \mathbb{Z}_{\geq 0}$ and $x \in \mathbf{U}_{q}^{-}$, we have

$$
\begin{aligned}
\left(\left(e_{i}^{\prime}\right)^{k}\left(D_{u, u^{\prime}}\right), x\right)_{L} & =\left(1-q_{i}^{2}\right)^{k}\left(D_{u, u^{\prime}}, f_{i}^{k} x\right)_{L} \\
& =\left(1-q_{i}^{2}\right)^{k}\left(u, f_{i}^{k} x \cdot u^{\prime}\right)_{\lambda}^{\varphi} \\
& =\left(1-q_{i}^{2}\right)^{k}\left(e_{i}^{k} \cdot u, x \cdot u^{\prime}\right)_{\lambda}^{\varphi}=\left(1-q_{i}^{2}\right)^{k}\left(D_{e_{i}^{k} \cdot u, u^{\prime}}, x\right)_{L} .
\end{aligned}
$$

Hence $\left(e_{i}^{\prime}\right)^{k}\left(D_{u, u^{\prime}}\right)=\left(1-q_{i}^{2}\right)^{k} D_{e_{i}^{k} \cdot u, u^{\prime}}$. Combining this equality with (8.1), we obtain the first equality. The second equality is proved in the same manner. The last two equalities are deduced from Proposition 3.22 and 3.46 .

Proof of Theorem 8.1. It is easily seen that we need only check the case that $x \in \mathbf{U}_{q}^{-}$. For $i \in I$, we have $D_{s_{i} \varpi_{i}, \varpi_{i}}=\left(1-q_{i}^{2}\right) f_{i}$. We first consider the images of $D_{s_{i} \varpi_{i}, \varpi_{i}}, i \in I$ under iterated application of $\eta_{w_{0}, q}$. If $I=\{i\}$, that is, $\mathfrak{g}=\mathfrak{s l}_{2}$, the quantum unipotent cell $\mathbf{A}_{q}\left[N_{-}^{w_{0}}\right]$ is generated by $D_{s_{i} \varpi_{i}, \varpi_{i}}^{ \pm 1}\left(=D_{w_{0} \varpi_{i}, \varpi_{i}}^{ \pm 1}\right)$. In this case, $\eta_{w_{0}, q}^{2}\left(D_{s_{i} \varpi_{i}, \varpi_{i}}\right)=D_{s_{i} \varpi_{i}, \varpi_{i}}$. Hence $\eta_{w_{0}, q}^{2}=\mathrm{id}$, in particular, the theorem holds. Henceforth, we consider the case that $\mathfrak{g}$ does not have ideals of Lie algebras which are isomorphic to $\mathfrak{s l}_{2}$. We have

$$
\eta_{w_{0}, q}\left(D_{s_{i} \varpi_{i}, \varpi_{i}}\right) \simeq D_{w_{0} \varpi_{i}, \varpi_{i}}^{-1} D_{w_{0} \varpi_{i}, s_{i} \varpi_{i}} .
$$

Here $\simeq$ stands for the coincidence up to some powers of $q$. Now, by Proposition 3.46, $D_{w_{0} \varpi_{i}, s_{i} \varpi_{i}}=G^{\mathrm{up}}\left(* \bar{\jmath}_{w_{0} \varpi_{i}}^{\vee}\left(u_{s_{i} \varpi_{i}}\right)\right)$. By Lemma 8.3 ,

$$
\varepsilon_{j}^{*}\left(* \bar{\jmath}_{w_{0} \varpi_{i}}^{\vee}\left(u_{s_{i} \varpi_{i}}\right)\right)=\varepsilon_{j}\left(\bar{\jmath}_{w_{0} \varpi_{i}}^{\vee}\left(u_{s_{i} \varpi_{i}}\right)\right)=\varphi_{j}\left(u_{s_{i} \varpi_{i}}\right)= \begin{cases}-a_{j i} & \text { if } j \neq i, \\ 0 & \text { if } j=i .\end{cases}
$$

Therefore $\sum_{j \in I} \varepsilon_{j}^{*}\left(* \bar{\jmath}_{w_{0} \varpi_{i}}^{\vee}\left(u_{s_{i} \varpi_{i}}\right)\right) \varpi_{j}=\varpi_{i}+s_{i} \varpi_{i}\left(=: \lambda_{1}\right)$. Recall Remark 6.4. Then there exists $b_{1} \in \mathscr{B}\left(\lambda_{1}\right)$ such that $D_{w_{0} \varpi_{i}, s_{i} \varpi_{i}}=D_{G_{\lambda_{1}}^{\text {up }}\left(b_{1}\right), u_{\lambda_{1}}}$, that is, $\bar{\jmath}_{\lambda_{1}}\left(b_{1}\right)=* \bar{\jmath}_{w_{0} \varpi_{i}}^{\vee}\left(u_{s_{i} \varpi_{i}}\right)$. Then

$$
\eta_{w_{0}, q}^{2}\left(D_{s_{i} \varpi_{i}, \varpi_{i}}\right) \simeq D_{w_{0} \varpi_{i}, \varpi_{i}} D_{w_{0} \lambda_{1}, \lambda_{1}}^{-1} D_{u_{w_{0} \lambda_{1}}, G^{\mathrm{up}}\left(b_{1}\right)} .
$$

As above, $D_{w_{0} \lambda_{1}, G_{\lambda_{1}}^{\text {up }}\left(b_{1}\right)}=G^{\text {up }}\left(* \bar{\jmath}_{w_{0} \lambda_{1}}^{\vee}\left(b_{1}\right)\right)$, and by Lemma 8.3 ,

$$
\begin{aligned}
\varepsilon_{j}^{*}\left(* \bar{\jmath}_{w_{0} \lambda_{1}}^{\vee}\left(b_{1}\right)\right) & =\varepsilon_{j}\left(\bar{\jmath}_{w_{0} \lambda_{1}}^{\vee}\left(b_{1}\right)\right) \\
& =\varepsilon_{j}\left(b_{1}\right)+\left\langle h_{j}, \text { wt } b_{1}\right\rangle \\
& =\varepsilon_{j}\left(\bar{\jmath}_{\lambda_{1}}\left(b_{1}\right)\right)+\left\langle h_{j}, w_{0} \varpi_{i}-s_{i} \varpi_{i}+\lambda_{1}\right\rangle \\
& =\varepsilon_{j}\left(* \bar{\jmath}_{w_{0} \varpi_{i}}^{\vee}\left(u_{s_{i} \varpi_{i}}\right)\right)+\left\langle h_{j}, w_{0} \varpi_{i}+\varpi_{i}\right\rangle .
\end{aligned}
$$

By Proposition 3.46 and Lemma 8.3 ,

$$
\varepsilon_{j}\left(* \bar{\jmath} w_{w_{0} \varpi_{i}}^{\vee}\left(u_{s_{i} \varpi_{i}}\right)\right)=\max \left\{k \in \mathbb{Z}_{\geq 0} \mid D_{e_{j}^{k} \cdot u_{w_{0} \varpi_{i}}, u_{s_{i} \varpi_{i}}} \neq 0\right\} .
$$

By the way, there is an involution $\theta$ on $I$ defined by $w_{0} \alpha_{i}=-\alpha_{\theta(i)}$. Then $w_{0} \varpi_{i}=-\varpi_{\theta(i)}$ and $s_{\theta(i)} w_{0} \varpi_{i}=w_{0} s_{i} \varpi_{i}$. When $\mathfrak{g}$ does not have ideals of Lie algebras which are isomorphic to $\mathfrak{s l}_{2}$, we have $D_{w_{0} s_{i} \varpi_{i}, s_{i} \varpi_{i}} \neq 0$. Therefore $\varepsilon_{j}\left(* \bar{j}_{w_{0} \varpi_{i}}^{\vee}\left(u_{s_{i} \varpi_{i}}\right)\right)=\delta_{j, \theta(i)}$. Hence

$$
\varepsilon_{j}^{*}\left(* \bar{\jmath}_{w_{0} \lambda_{1}}^{\vee}\left(b_{1}\right)\right)=\delta_{j, \theta(i)}-\delta_{j, \theta(i)}+\delta_{i j}=\delta_{i j} .
$$


Therefore $\sum_{j \in I} \varepsilon_{j}^{*}\left(* \bar{\jmath}_{w_{0} \lambda_{1}}^{\vee}\left(b_{1}\right)\right) \varpi_{j}=\varpi_{i}$. Then there exists $b_{2} \in \mathscr{B}\left(\varpi_{i}\right)$ such that $D_{w_{0} \lambda_{1}, G_{\lambda_{1}}^{\mathrm{up}}\left(b_{1}\right)}=$ $D_{G_{\varpi_{i}}^{\mathrm{up}}\left(b_{2}\right), u_{\varpi_{i}}}$. Then

$$
\begin{aligned}
\eta_{w_{0}, q}^{3}\left(D_{s_{i} \varpi_{i}, \varpi_{i}}\right) & \simeq D_{w_{0} \varpi_{i}, \varpi_{i}}^{-1} D_{w_{0} \lambda_{1}, \lambda_{1}} D_{w_{0} \varpi_{i}, \varpi_{i}}^{-1} D_{u_{w_{0} \varpi_{i}}, G_{\varpi_{i}}^{\mathrm{up}_{i}}\left(b_{2}\right)} \\
& \simeq D_{w_{0},-\alpha_{i}} D_{u_{w_{0} \varpi_{i}}, G_{\varpi_{i}}}^{\mathrm{up}_{(}\left(b_{2}\right)} .
\end{aligned}
$$

Here,

$$
\text { wt } \begin{aligned}
D_{u_{w_{0} \varpi_{i}}, G_{\varpi_{i}}^{\text {up }}\left(b_{2}\right)} & =w_{0} \varpi_{i}-\text { wt } b_{2}=w_{0} \varpi_{i}-\left(w_{0} \lambda_{1}-\text { wt } b_{1}+\varpi_{i}\right) \\
& =w_{0} \varpi_{i}-\left(w_{0} \lambda_{1}-\left(w_{0} \varpi_{i}-s_{i} \varpi_{i}+\lambda_{1}\right)+\varpi_{i}\right)=-\alpha_{\theta(i)} .
\end{aligned}
$$

Hence $D_{u_{w_{0} \varpi_{i}}, G_{\varpi_{i}}^{\text {up }}\left(b_{2}\right)}=D_{s_{\theta(i)} \varpi_{\theta(i)}, \varpi_{\theta(i)}}$ because both sides are unique elements of the dual canonical basis of weight $-\alpha_{\theta(i)}$. Therefore,

$$
\eta_{w_{0}, q}^{6}\left(D_{s_{i} \varpi_{i}, \varpi_{i}}\right) \simeq D_{w_{0}, \alpha_{i}-\alpha_{\theta(i)}} D_{s_{i} \varpi_{i}, \varpi_{i}} .
$$

Moreover, by Theorem 6.1, $\eta_{w_{0}, q}^{6}\left(D_{s_{i} \varpi_{i}, \varpi_{i}}\right)$ is an element of dual canonical basis, in particular, dual bar-invariant. Therefore,

$$
\eta_{w_{0}, q}^{6}\left(D_{s_{i} \varpi_{i}, \varpi_{i}}\right)=q^{\left(\alpha_{i}-\alpha_{\theta(i)}, \alpha_{i}\right)} D_{w_{0}, \alpha_{i}-\alpha_{\theta(i)}} D_{s_{i} \varpi_{i}, \varpi_{i}} .
$$

By this result and Proposition 4.2, 4.7, for $i_{1}, \ldots, i_{\ell} \in I$, we have

$$
\begin{aligned}
& \eta_{w_{0}, q}^{6}\left(D_{s_{i_{1}} \varpi_{i_{1}}, \varpi_{i_{1}}} \cdots D_{s_{i_{\ell}} \varpi_{i_{\ell}}, \varpi_{i_{\ell}}}\right) \\
& =q^{\sum_{k=1}^{\ell}\left(\alpha_{i_{k}}-\alpha_{\theta\left(i_{k}\right)}, \alpha_{i_{k}}\right)} D_{w_{0}, \alpha_{i_{1}}-\alpha_{\theta\left(i_{1}\right)}} D_{s_{i_{1}} \varpi_{i_{1}}, \varpi_{i_{1}}} \cdots D_{w_{0}, \alpha_{i_{\ell}}-\alpha_{\theta\left(i_{\ell}\right)}} D_{s_{i_{\ell}} \varpi_{i_{\ell}}, \varpi_{i_{\ell}}} \\
& =q^{\left(\sum_{k=1}^{\ell} \alpha_{i_{k}}-\sum_{k=1}^{\ell} \alpha_{\theta\left(i_{k}\right)}, \sum_{k=1}^{\ell} \alpha_{i_{k}}\right)} D_{w_{0}, \sum_{k=1}^{\ell} \alpha_{i_{k}}-\sum_{k=1}^{\ell} \alpha_{\theta\left(i_{k}\right)}} D_{s_{i_{1}} \varpi_{i_{1}}, \varpi_{i_{1}}} \cdots D_{s_{i_{\ell}} \varpi_{i_{\ell}}, \varpi_{i_{\ell}}} .
\end{aligned}
$$

Hence the desired equality in the theorem holds for all $x \in \mathbf{A}_{q}\left[N_{-}\right]$since the elements $D_{s_{i} \varpi_{i}, \varpi_{i}}=\left(1-q_{i}^{2}\right) f_{i}, i \in I$ generate the quantum unipotent subgroup $\mathbf{A}_{q}\left[N_{-}\right]$. Then we can easily extend this result to that for $\mathbf{A}_{q}\left[N_{-}^{w_{0}}\right]$ by straightforward calculation. The explicit calculation is left to the reader.

Remark 8.4. In the essential part of the proof of Theorem 8.1, we check the periodicity on generators of $\mathbf{A}_{q}\left[N_{-}^{w_{0}}\right]$. We should note that this set of generators is not the set of generators of $\mathbb{C}\left[N_{-}^{w_{0}}\right]$ after specialization unless $\mathfrak{g}=\mathfrak{s l}_{2}$.

Indeed, in general Kac-Moody cases, the quantum unipotent cell $\mathbf{A}_{q}\left[N_{-}^{w}\right]$ is generated by $\left\{\left[f_{i}\right] \mid i \in I\right\} \cup\left\{\left[D_{w \rho, \rho}\right]^{-1}\right\}$ (recall that $\rho:=\sum_{i \in I} \varpi_{i}$ ). In particular,

(the number of the generators of $\left.\mathbf{A}_{q}\left[N_{-}^{w}\right]\right) \leq \# I+1$.

On the other hand,

(the number of the generators of $\left.\mathbb{C}\left[N_{-}^{w}\right]\right) \geq \operatorname{dim} N_{-}^{w}=\ell(w)$.

Therefore the periodicity might be checked in the quantum setting more easily than in the classical setting by this decrease of numbers of generators.

Acknowledgement. The authors are grateful to Bernard Leclerc for his enlightening advice concerning cluster algebras and their categorifications. They would like to express our sincere gratitude to Yoshihisa Saito, the supervisor of the second author, for his helpful comments. They wish to thank Ryo Sato and Bea Schumann for several interesting comments and discussions. They are also thankful to the anonymous referees whose suggestions significantly 
improve the present paper. The second author thanks the University of Caen Normandy, where a part of this paper was written, for the hospitality.

\section{REFERENCES}

[AR96] M. Auslander and I. Reiten, DTr-periodic modules and functors, Representation theory of algebras (Cocoyoc, 1994), 39-50, CMS Conf. Proc., 18, Amer. Math. Soc., Providence, RI, 1996.

[BCP99] J. Beck, V. Chari, and A. Pressley, An algebraic characterization of the affine canonical basis, Duke Math. J. 99 (1999), no. 3, 455-487.

[BFZ96] A. Berenstein, S. Fomin, and A. Zelevinsky, Parametrizations of canonical bases and totally positive matrices, Adv. Math. 122 (1996), no. 1, 49-149.

[BFZ05] — Cluster algebras. III. Upper bounds and double Bruhat cells, Duke Math. J. 126 (2005), no. $1,1-52$.

[BIRS09] A. B. Buan, O. Iyama, I. Reiten, and J. Scott, Cluster structures for 2-Calabi-Yau categories and unipotent groups, Compos. Math. 145 (2009), no. 4, 1035-1079.

[BR15] A. Berenstein and D. Rupel, Quantum cluster characters of Hall algebras, Selecta Math. (N.S.) 21 (2015), no. 4, 1121-1176.

[BZ93] A. Berenstein and A. Zelevinsky, String bases for quantum groups of type $A_{r}$, I. M. Gel'fand Seminar, 51-89, Adv. Soviet Math., 16, Part 1, Amer. Math. Soc., Providence, RI, 1993.

[BZ96] _ Canonical bases for the quantum group of type $A_{r}$ and piecewise-linear combinatorics, Duke Math. J. 82 (1996), no. 3, 473-502.

[BZ97] _ Total positivity in Schubert varieties, Comment. Math. Helv. 72 (1997), no. 1, $128-166$.

[BZ05] — Quantum cluster algebras, Adv. Math. 195 (2005), no. 2, 405-455.

[BZ14] _ Triangular bases in quantum cluster algebras, Int. Math. Res. Not. IMRN (2014), no. 6, 16511688.

[CB00] W. Crawley-Boevey, On the exceptional fibres of Kleinian singularities, Amer. J. Math. 122 (2000), no. $5,1027-1037$.

[DKP95] C. De Concini, V. G. Kac, and C. Procesi, Some quantum analogues of solvable Lie groups, Geometry and analysis (Bombay, 1992), 41-65, Tata Inst. Fund. Res., Bombay, 1995.

[DP97] C. De Concini and C. Procesi, Quantum Schubert cells and representations at roots of 1 , Algebraic groups and Lie groups, 127-160, Austral. Math. Soc. Lect. Ser., 9, Cambridge Univ. Press, Cambridge, 1997.

[Dri87] V. G. Drinfel'd, Quantum groups, Proceedings of the International Congress of Mathematicians, Vol. 1, 2 (Berkeley, Calif., 1986), 798-820, Amer. Math. Soc., Providence, RI, 1987.

[ES98] K. Erdmann and N. Snashall, Preprojective algebras of Dynkin type, periodicity and the second Hochschild cohomology, Algebras and modules, II (Geiranger, 1996), 183-193, CMS Conf. Proc., 24, Amer. Math. Soc., Providence, RI, 1998.

[FZ99] S. Fomin and A. Zelevinsky, Double Bruhat cells and total positivity, J. Amer. Math. Soc. 12 (1999), no. $2,335-380$.

[FZ02] _ Cluster algebras. I. Foundations, J. Amer. Math. Soc. 15 (2002), no. 2, 497-529.

[GLS05] C. Geiß, B. Leclerc, and J. Schröer, Semicanonical bases and preprojective algebras, Ann. Sci. École Norm. Sup. (4) 38 (2005), no. 2, 193-253.

[GLS07a] _ Cluster algebra structures and semicanonical bases for unipotent groups, arXiv preprint, arXiv:math/0703039, 2007.

[GLS07b] , Semicanonical bases and preprojective algebras. II. A multiplication formula, Compos. Math. 143 (2007), no. 5, 1313-1334.

[GLS11] _ Kac-Moody groups and cluster algebras, Adv. Math. 228 (2011), no. 1, 329-433.

[GLS12] _ Generic bases for cluster algebras and the Chamber ansatz, J. Amer. Math. Soc. 25 (2012), no. $1,21-76$.

[GLS13] — Cluster structures on quantum coordinate rings, Selecta Math. (N.S.) 19 (2013), no. 2, 337397.

[GW04] K. R. Goodearl and R. B. Warfield, Jr., An introduction to noncommutative Noetherian rings, Second edition. London Mathematical Society Student Texts, 61. Cambridge University Press, Cambridge, 2004. xxiv $+344 \mathrm{pp}$. 
[GY14] K. R. Goodearl and M. T. Yakimov, Quantum cluster algebras and quantum nilpotent algebras, Proc. Natl. Acad. Sci. USA 111 (2014), no. 27, 9696-9703.

[GY16] , The Berenstein-Zelevinsky quantum cluster algebra conjecture, arXiv preprint, arXiv:1602.00498, 2016. To appear in J. Eur. Math. Soc. (JEMS).

[GY17] , Quantum cluster algebra structures on quantum nilpotent algebras, Mem. Amer. Math. Soc. 247 (2017), no. 1169, vii+119 pp.

[Hum90] J. E. Humphreys, Reflection groups and Coxeter groups, Cambridge Studies in Advanced Mathematics, 29. Cambridge University Press, Cambridge, 1990. xii+204 pp.

[Jos95] A. Joseph, Quantum groups and their primitive ideals, Ergebnisse der Mathematik und ihrer Grenzgebiete (3) [Results in Mathematics and Related Areas (3)], 29, Springer-Verlag, Berlin, 1995. x+383 pp.

[Kas89] M. Kashiwara, The flag manifold of Kac-Moody Lie algebra, Algebraic analysis, geometry, and number theory (Baltimore, MD, 1988), 161-190, Johns Hopkins Univ. Press, Baltimore, MD, 1989.

[Kas91] _ On crystal bases of the Q-analogue of universal enveloping algebras, Duke Math. J. 63 (1991), no. $2,465-516$.

[Kas93a] _ The crystal base and Littelmann's refined Demazure character formula, Duke Math. J. 71 (1993), no. 3, 839-858.

[Kas93b] _ Global crystal bases of quantum groups, Duke Math. J. 69 (1993), no. 2, 455-485.

[Kas94] _ C Crystal bases of modified quantized enveloping algebra, Duke Math. J. 73 (1994), no. 2, 383413.

[Kas95] _ On crystal bases, Representations of groups (Banff, AB, 1994), 155-197, CMS Conf. Proc., 16, Amer. Math. Soc., Providence, RI, 1995.

[Kim12] Y. Kimura, Quantum unipotent subgroup and dual canonical basis, Kyoto J. Math. 52 (2012), no. 2, $277-331$.

[KKKO18] S.-J. Kang, M. Kashiwara, M. Kim, and S-j. Oh, Monoidal categorification of cluster algebras, J. Amer. Math. Soc. 31 (2018), no. 2, 349-426.

[KO18] Y. Kimura and H. Oya, Quantum twist maps and dual canonical bases, Algebr. Represent. Theory 21 (2018), no. 3, 589-604.

[KP83] V. G. Kac and D. H. Peterson, Regular functions on certain infinite-dimensional groups, Arithmetic and geometry, Vol. II, 141-166, Progr. Math., 36, Birkhäuser Boston, Boston, MA, 1983.

[KT95] M. Kashiwara and T. Tanisaki, Kazhdan-Lusztig conjecture for affine Lie algebras with negative level, Duke Math. J. 77 (1995), no. 1, 21-62.

[Kum02] S. Kumar, Kac-Moody groups, their flag varieties and representation theory, Progress in Mathematics, 204, Birkhäuser Boston, Inc., Boston, MA, 2002. xvi+606 pp.

[Lus90a] G. Lusztig, Canonical bases arising from quantized enveloping algebras, J. Amer. Math. Soc. 3 (1990), no. 2, 447-498.

[Lus90b] _ Canonical bases arising from quantized enveloping algebras. II, Progr. Theoret. Phys. Suppl. (1990), no. 102, 175-201 (1991), Common trends in mathematics and quantum field theories (Kyoto, 1990).

[Lus91] _ Quivers, perverse sheaves, and quantized enveloping algebras, J. Amer. Math. Soc. 4 (1991), no. $2,365-421$.

[Lus94] _ Total positivity in reductive groups, Lie theory and geometry, 531-568, Progr. Math., 123, Birkhäuser Boston, Boston, MA, 1994.

[Lus00] — Semicanonical bases arising from enveloping algebras, Adv. Math. 151 (2000), no. 2, $129-139$.

[Lus10] Introduction to quantum groups, Reprint of the 1994 edition. Modern Birkhäuser Classics. Birkhäuser/Springer, New York, 2010. xiv+346 pp.

[LY15] T. H. Lenagan and M. T. Yakimov, Prime factors of quantum Schubert cell algebras and clusters for quantum Richardson varieties, arXiv preprint, arXiv:1503.06297, 2015. To appear in J. Reine Angew. Math.

[Oya17] H. Oya, The Chamber Ansatz for quantum unipotent cells, arXiv preprint, arXiv:1702.00383, 2017. To appear in Transform. Groups.

[Qin16] F. Qin, Compare triangular bases of acyclic quantum cluster algebras, arXiv preprint, arXiv:1606.05604, 2016. To appear in Trans. Amer. Math. Soc.

[Qin17] , Triangular bases in quantum cluster algebras and monoidal categorification conjectures, Duke Math. J. 166 (2017), no. 12, 2337-2442. 
[Tan92] T. Tanisaki, Killing forms, Harish-Chandra isomorphisms, and universal R-matrices for quantum algebras, Infinite analysis, Part A, B (Kyoto, 1991), 941-961, Adv. Ser. Math. Phys., 16, World Sci. Publ., River Edge, NJ, 1992.

[Tan17] _ Modules over quantized coordinate algebras and PBW-bases, J. Math. Soc. Japan 69 (2017), no. 3, 1105-1156.

[Wil13] H. Williams, Cluster ensembles and Kac-Moody groups, Adv. Math. 247 (2013), 1-40.

[Yak10] M. T. Yakimov, Invariant prime ideals in quantizations of nilpotent Lie algebras, Proc. Lond. Math. Soc. (3) 101 (2010), no. 2, 454-476.

(Yoshiyuki Kimura) Faculty of Liberal Arts and Sciences, Osaka Prefecture University, 1-1, GaKuen-Cho, NAKa-Ku, SAKai, Osaka 599-8531, JAPAN

E-mail address: ysykimura@las.osakafu-u.ac.jp

(Hironori Oya) Department of Mathematical Sciences, Shibaura Institute of Technology, 307 Fukasaku, Minuma-Ku, Saitama-shi, Saitama, 337-8570, Japan

E-mail address: hoya@shibaura-it.ac.jp 